\title{
Neural dynamics of attentionally modulated Pavlovian conditioning: Conditioned reinforcement, inhibition, and opponent processing
}

\author{
STEPHEN GROSSBERG and NESTOR A. SCHMAJUK \\ Boston University, Boston, Massachusetts
}

\begin{abstract}
A real-time neural network model is developed to explain data about the acquisition and extinction of conditioned excitors and inhibitors. Systematic computer simulations are described of a READ circuit, which joins together a mechanism of associative learning with an opponentprocessing circuit, called a recurrent gated dipole. READ circuit properties clarify how positive and negative reinforcers are learned and extinguished during primary and secondary conditioning. Habituating chemical transmitters within a gated dipole determine an affective adaptation level, or context, against which later events are evaluated. Neutral conditioned stimuli can become reinforcers by being associated either with direct activations or with antagonistic rebounds within a previously habituated dipole. Neural mechanisms are characterized whereby conditioning can be actively extinguished, and associative saturation prevented, by a process called opponent extinction, even if no passive memory decay occurs. Opponent extinction exploits a functional dissociation between read-in and read-out of associative memory, which may be achieved by locating the associative mechanism at dendritic spines. READ circuit mechanisms are joined to cognitive-emotional mechanisms for associative learning of conditioned reinforcers and of incentive motivation, and to cognitive-in particular, adaptive resonance theory-mechanisms for activating and storing internal representations of sensory cues in a limited-capacity short-term memory (STM); for learning, matching, and mismatching sensory expectancies, leading to the enhancement or updating of STM; and for shifting the focus of attention toward sensory representations whose reinforcement history is consistent with momentary appetitive requirements. This total neural architecture is used to explain conditioning and extinction of a conditioned excitor; conditioning and nonextinction of a conditioned inhibitor; and properties of conditioned inhibition as a "slave" process and as a "comparator" process, including effects of pretest deflation or inflation of the conditioning context, of familiar or novel training or test contexts, of weak or strong shocks, and of preconditioning unconditioned-stimulus-alone exposures. The same mechanisms have elsewhere been used to explain phenomena such as blocking, unblocking, overshadowing, latent inhibition, superconditioning, inverted $U$ in conditioning as a function of interstimulus interval, anticipatory conditioned responses, partial reinforcement acquisition effect, learned helplessness, and vicious-circle behavior. The theory clarifies why alternative models have been unable to explain an equally large data base.
\end{abstract}

\section{Introduction: The Analysis of \\ Distributed Neural Architectures}

A key problem in biological theories of intelligence concerns the manner in which external events interact with internal organismic requirements to focus attention upon motivationally desired goals. The present work further develops a theory that involves sensory-cognitive and

S. Grossberg was supported in part by the Air Force Office of Scientific Research (AFOSR 85-0149 and AFOSR F49620-86-C-0037), the Army Research Office (ARO DAAG-29-85-K-0095), and the National Science Foundation (NSF IRI-84-17756). N. A. Schmajuk was supported in part by the National Science Foundation (NSF IRI-84-17756). The authors wish to thank Cynthia Suchta and Carol Yanakakis for their valuable assistance in the preparation of the manuscript and illustrations. The authors' mailing address is Center for Adaptive Systems, Boston University, 111 Cunningham Street, Boston, MA 02215. cognitive-reinforcement circuits. The theory is applied to the explanation of data about the acquisition and extinction of classically conditioned excitors and inhibitors.

The neural architectures that are engaged during classical conditioning are distributed across several brain regions. Even the relatively simple architecture that controls the rabbit's nictitating membrane response includes such widely separated regions as the cerebellum (McCormick \& Thompson, 1984) and hippocampus (Berger \& Thompson, 1978). In order to understand the workings of such a distributed neural architecture, one must simultaneously analyze both the whole and its parts, both the macroscopic and the microscopic description of the architecture. On the macroscopic level, one needs to understand the functional and computational relationships that clarify how the architecture controls a particular class of 
behaviors, and why it is composed of particular types of circuits. On the microscopic level, one needs to attain a detailed analytic understanding of how each circuit is designed, of how it works, and of what types of mechanistic variations can be expected to occur as evolutionary variations across species. These macroscopic functional analyses and microscopic mechanistic analyses are, moreover, not independent, because the behavioral properties controlled by a neural architecture are typically emergent properties that arise from interactions among its component circuits.

A thoroughly characterized neural architecture provides an explicit real-time description of how behaviorally observable stimuli influence the internal neural transformations that regulate behaviorally observable responses. Although a number of extremely useful phenomenological and formal conditioning models have been described during the past two decades, most of these models do not provide an explicit description of the real-time mechanisms that would be required to instantiate their concepts and equations. In particular, although the classical Rescorla and Wagner (1972) model is formulated in terms of difference equations whose variables change through time, these equations do not provide an explicit characterization of real-time mechanisms that might be able to instantiate the model's properties. Such an attempt at explication shows, moreover, that the Rescorla-Wagner model cannot be consistently embedded into an explicit real-time model (Grossberg, 1982a).

The demand for a real-time processing description has been shown to impose important design constraints upon the formulation of conditioning processes. The advantage of a real-time theory can be appreciated through examples of how such a theory has articulated the coordinated neural events that are triggered by unexpected changes in external environmental contingencies, notably by the unexpected nonoccurrence of a primary or conditioned reinforcer, or by unexpected changes in contingent or noncontingent probabilities of a conditioned stimulus (CS) or unconditioned stimulus (US) in a given experimental context.

At least two types of neural network macrocircuits are needed to provide a real-time explanation of a broad range of data about the acquisition and extinction of conditioned excitors and inhibitors.

Sensory-cognitive circuit. Sensory-cognitive interactions in the theory are carried out by an adaptive resonance theory (ART) circuit (Carpenter \& Grossberg, 1985, 1987; Grossberg, 1976b, 1987a). The ART architecture suggests how internal representations of sensory events, including CSs and USs, can be learned in stable fashion (Figure 1). Among the mechanisms used for stable self-organization of sensory recognition codes are the top-down expectations that are matched against bottomup sensory signals. When a mismatch occurs, an orienting arousal burst acts to reset the sensory representation of all cues that are currently being stored in short-term
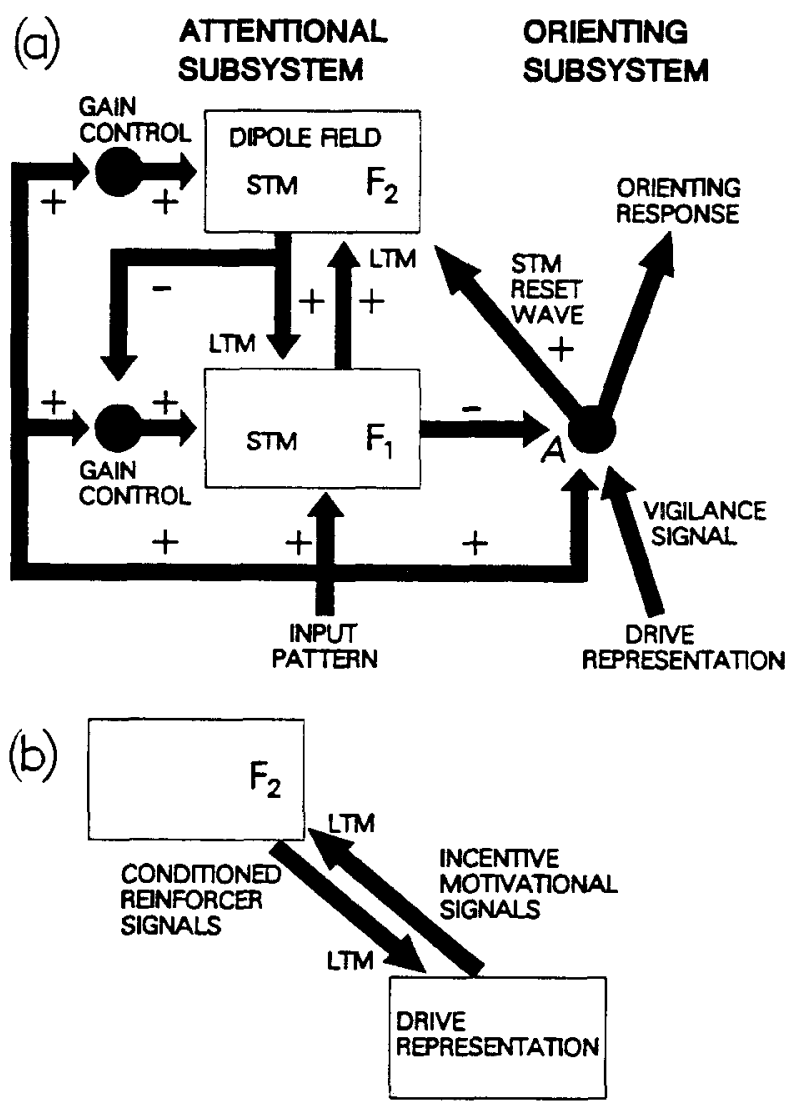

Figure 1. Anatomy of an adaptive resonance theory (ART) circuit. (a) Interactions between the attentional and orienting subsystems: Code learning takes place at the long-term memory (LTM) traces within the bottom-up and top-down pathways between levels $F_{1}$ and $F_{2}$. The top-down pathways can read out learned expectations, or templates, that are matched against bottom-up input patterns at $F_{1}$. Mismatches activate the orienting subsystem $A$, thereby resetting short-term memory (STM) at $F_{2}$ and initiating search for another recognition code. Subsystem $A$ can also activate an orienting response. Sensitivity to mismatch at $F_{1}$ is modulated by vigilance signals from drive representations. (b) Trainable pathways exist between level $F_{2}$ and the drive representations. Learning from $F_{2}$ to a drive representation endows a recognition category with conditioned reinforcer properties. Learning from a drive representation to $F_{2}$ associates the drive representation with a set of motivationally compatible categories. (From 'Neural Dynamics of Category Learning and Recognition: Structural Invariants, Reinforcement, and Evoked Potentials," by G. A. Carpenter and S. Grossberg, in press, Quantitative Analyses of Behavior, Vol. 8. Printed by permission.)

memory (STM). In particular, representations with high STM activation tend to become less active, representations with low STM activation tend to become more active, and the novel event that caused the mismatch tends to be more actively stored than it would have been had it been expected.

Cognitive-reinforcement circuit. Cognitive-reinforcer interactions in the theory are carried out in the circuit described in Figure 2. In this circuit, there exist cell populations that are separate from sensory representations and 


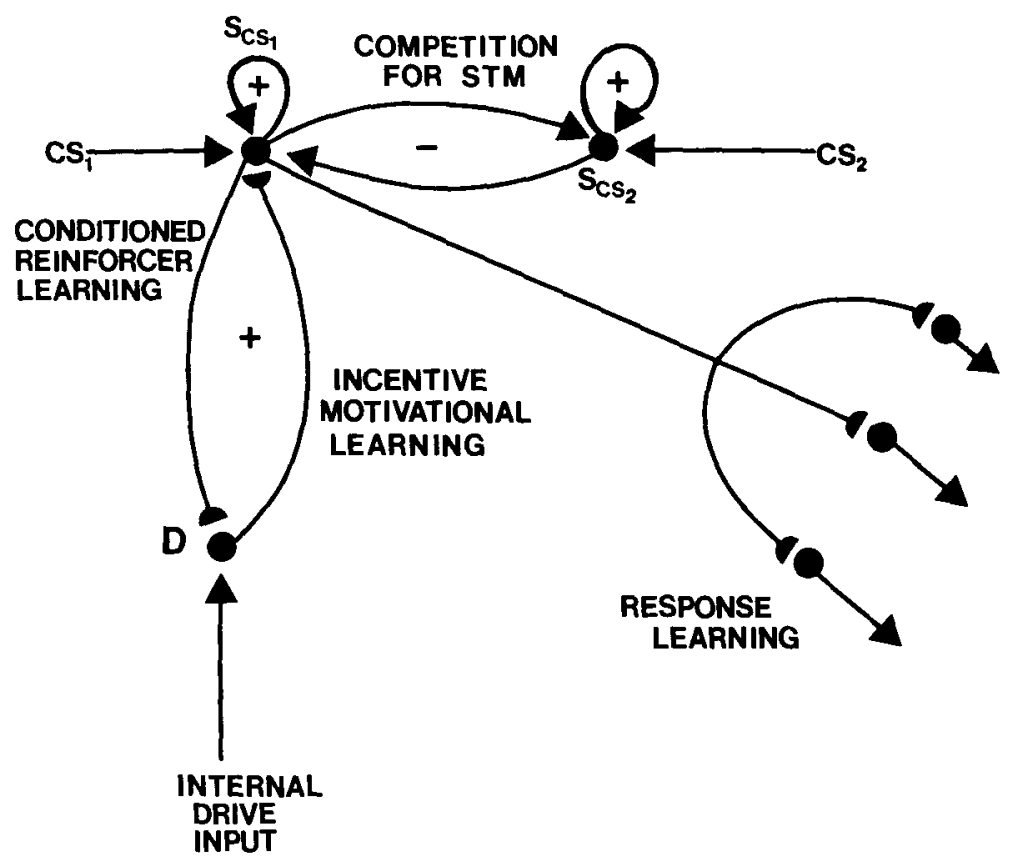

Figure 2. Schematic conditioning circuit. Conditioned stimuli activate sensory representations ( $\mathrm{SCS}_{j}$ ), which compete among themselves for limited-capacity short-term memory activation and storage. The activated $\mathrm{SCs}_{i}$ elicit conditionable signals to drive representations and motor command representations. Learning from an $S_{C s}$ to a drive representation, D, is called conditioned reinforcer learning. Learning from D to an $\mathrm{Scs}_{\text {, }}$ is called incentive motivational learning. Signals from D to $\operatorname{Scs}_{\text {, }}$ are elicited when the combination of external sensory inputs plus internal drive inputs is sufinciently large. In the simulations reported herein, the drive level is assumed to be large and constant.

related to particular drives and motivational variables (Grossberg, 1972a, 1987a). Repeated pairing of a CS sensory representation, $S_{C S}$, with activation of a drive representation, $\mathrm{D}$, by a reinforcer causes the modifiable synapses connecting $\mathrm{S}_{\mathrm{CS}}$ with $\mathrm{D}$ to become strengthened. Incentive motivation pathways from the drive representations to the sensory representations are also assumed to be conditionable. These conditioned $S \rightarrow D \rightarrow S$ feedback pathways shift the attentional focus to the set of previously reinforced, motivationally compatible cues (Figure 2). This shift of attention occurs because the sensory representations, which emit conditioned reinforcer signals and receive conditioned incentive motivation signals, compete among themselves for a limited-capacity STM via on-center off-surround interactions. When incentive motivational feedback signals are received at the sensory representational field, these signals can bias the competition for STM activity toward motivationally salient cues.

In order to explain the moment-by-moment dynamics of conditioning, an additional microcircuit must be embedded in the drive representations of the macrocircuit depicted in Figure 2. This microcircuit, called a gated dipole (Grossberg, 1972a, 1972b), instantiates a neurophysiological theory of opponent processing. The need for a certain type of opponent processing for conditioning circuits can be seen from the following considerations.
The gated-dipole opponent process. In the cognitivereinforcement circuit, CSs are conditioned to either the onset or the offset of a reinforcer. For example, a CS that is conditioned to the onset of a shock can become a source of conditioned fear (excitor). A CS that is conditioned to the offset of a shock can become a source of conditioned relief (inhibitor). A gated-dipole opponent process explains how the offset of a reinforcer can generate an offresponse, or antagonistic rebound, to which a simultaneous CS can be conditioned. A gated dipole is a minimal neural-network opponent process that is capable of generating a sustained, but habituative, on-response (e.g., a fear reaction) to onset of a cue (e.g., a shock), as well as a transient off-response (e.g., a relief reaction), or antagonistic rebound, to offset of the cue.

The READ circuit: A synthesis of opponent processing and associative learning mechanisms. A specialized gated-dipole circuit is needed to explain phenomena such as secondary inhibitory conditioning. Secondary inhibitory conditioning consists of two phases. In Phase $1, \mathbf{C S}_{1}$ becomes an excitatory conditioned reinforcer (e.g., a source of conditioned fear) by being paired with a US (e.g., a shock). In Phase 2, the offset of $\mathrm{CS}_{1}$ can generate an off-response that can condition a subsequent $\mathbf{C S}_{2}$ to become an inhibitory conditioned reinforcer (e.g., a source of conditioned relief). In order to explain secondary inhibitory conditioning, a gated-dipole circuit must also contain internal feedback pathways; that is, it should 
be recurrent. In addition, such a recurrent gated dipole must be joined to a mechanism of associative learning, whereby CSs may become conditioned excitors or inhibitors. A circuit design that realizes all these properties is called a READ circuit, as a mnemonic for REcurrent Associative gated Dipole (Figure 3).

The design of the READ circuit clarifies many properties of conditioning data; for example, how the extinction of conditioned excitors and the nonextinction of conditioned inhibitors may be explained by a single neural circuit (Part II). Our analysis links these data properties to the functional property that opponent interactions actively cause extinction even in cases in which passive extinction does not occur. In the absence of passive extinction, an associative memory could easily saturate. Opponent extinction shows how saturation is prevented and active extinction obtained even if no passive memory decay occurs. These functional properties of opponent extinction can be achieved using a mechanistic property that dissociates the read-in and read-out of associative memory. Such a mechanistic property can, in turn, be realized by locating associative synapses on dendritic spines.

Thus, the present article describes two related types of results. Part I provides a quantitative computational analysis of several variants of a READ circuit design that forms part of the total neural architecture for the control of classically and operantly conditionable behaviors that is schematized in Figures 1-3. Based upon the mathematical theory of classical conditioning and associative learning that was provided in Grossberg (1969a, 1969c, 1970), the development of this architecture began in Grossberg

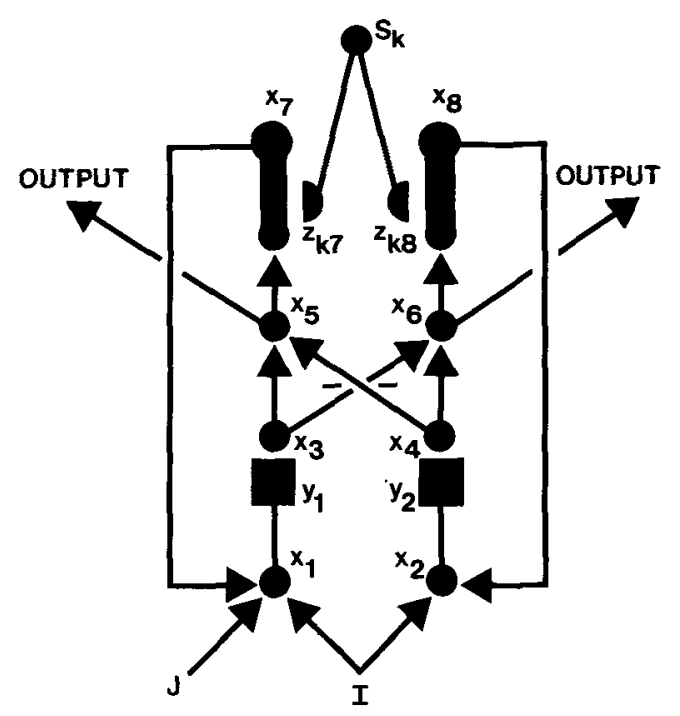

Figure 3. A READ I circuit. This circuit joins together a recurrent gated dipole with an associative learning mechanism. Learning is driven by signals, $S_{k}$, from sensory representations, $S_{k}$, which activate long-term memory (LTM) traces $z_{k 7}$ and $z_{k 8}$, which sample activation levels at the on-channel and off-channel, respectively, of the gated dipole. See text for details.
(1971). Since that time, the component circuits of the architecture have been progressively elaborated in a series of articles aimed at explaining and predicting an everlarger behavioral, psychophysiological, neurophysiological, anatomical, and neuropharmacological data base about conditioning and its control mechanisms. Key accomplishments of this theory during its first decade of existence are reviewed and further development provided in Grossberg (1982a, 1982b, 1984b). These recent articles are gathered together in Grossberg (1987a).

The READ circuit design analyzed in Part $I$ is one of several specialized gated-dipole circuits that have been identified through the parametric analysis of behavioral and brain data. Specialized gated-dipole circuits have also played a key role in helping to explain and predict a wide variety of other data bases. ${ }^{1}$ Thus, a gated-dipole circuit may at this time be said with some confidence to instantiate a basic principle of neural design.

Involvement of a gated-dipole circuit can be inferred from behavioral data through its characteristic constellation, or bundle, of emergent properties. These include mutually dependent properties of habituation, antagonistic rebound, adaptation-level processing, and an inverted $U$ in sensitivity. The habituative, antagonisticrebound, and adaptation-level properties play an important role in the applications described herein. Antagonisticrebound properties are particularly important for understanding how a real-time theory can fill the conceptual gaps left by a merely formal theory. This is because antagonistic-rebound reactions often occur subsequent to the offset of an external cue or subsequent to the nonoccurrence of an expected cue. Such antagonistic rebounds thus occur during time intervals when no experimentally controlled external cues are active. Only in a real-time theory can such reactions be explained without invoking ad hoc hypotheses. Indeed, the very existence, no less than the size, of an antagonistic rebound can depend upon a host of contextual and learning-dependent factors, whose properties can be conveniently analyzed in a real-time theory but not in a merely formal theory. Since such rebounds influence key observable properties of behavioral conditioning and extinction, formal theories are fundamentally limited in their ability to explain data in which the spatiotemporal organization of CSs and USs mixes together learnable direct reactions and antagonistic rebounds to these cues.

Part II joins the results of Part I to the other real-time circuits of the neural architecture schematized in Figures 1 and 2 to explain conditioning data. These include mechanisms for activating and storing internal representations of sensory cues in a limited-capacity working memory, or STM; mechanisms for learning, matching, and mismatching of sensory expectancies, leading to the enhancement or updating of STM processing; and mechanisms for shifting the focus of attention toward sensory representations whose reinforcement history is consistent with momentary appetitive requirements. This total architecture is then used to qualitatively explain the important data 
concerning acquisition and extinction of conditioned excitation and inhibition obtained by Lysle and Fowler (1985) and Miller and Schachtman (1985), as well as the results of many other related studies. Along the way, the qualitative explanatory concepts of these authors are explicated, refined, and generalized, and related data about such phenomena as blocking are also analyzed in a unified fashion.

\section{PART I}

\section{Simulating the Mechanism of Conditioned Reinforcement}

In Part I, computer simulations are used to characterize the behavior of a circuit in which a gated dipole is joined to a mechanism of Pavlovian conditioning. Multiple neural circuits are conditioned during a typical conditioning experiment. The conditioning events described in Part I constitute the type of learning whereby a CS becomes a conditioned reinforcer by being paired with a US. Both primary conditioning and secondary conditioning, as well as excitatory conditioning and inhibitory conditioning, are demonstrated.

In the simulations of primary excitatory conditioning, a conditioned stimulus $\left(\mathrm{CS}_{1}\right)$ precedes the onset of a US, as in Figure $4 \mathrm{a}$. As a result of conditioning, the $C S_{1}$ becomes a conditioned reinforcer with the same motivational sign as the US. In the simulations of secondary conditioning, another conditioned stimulus $\left(\mathrm{CS}_{2}\right)$ precedes a conditioned reinforcer (the $\mathrm{CS}_{1}$ of a primary conditioning experiment), as in Figure $4 \mathrm{~b}$. As a result of conditioning, the $\mathrm{CS}_{2}$ also becomes a conditioned reinforcer with the same motivational sign as the $C_{1}$. In primary inhibitory conditioning, a $\mathrm{CS}_{1}$ occurs subsequent to the offset of a US, as in Figure 4c. As a result of conditioning, the $\mathrm{CS}_{1}$ becomes a conditioned reinforcer whose motivational sign is the opposite of that of the US. In secondary inhibitory conditioning, a $\mathrm{CS}_{2}$ occurs subsequent to the offset of a conditioned reinforcer $\left(\mathrm{CS}_{1}\right)$, as in Figure $4 \mathrm{~d}$. As a result of conditioning, the $\mathrm{CS}_{2}$ becomes a conditioned reinforcer whose motivational sign is the opposite of that of the $\mathrm{CS}_{1}$. In addition, we investigate how these several types of conditioned reinforcer learning can extinguish if the CSs are presented without reinforcement on subsequent trials.

Many variations of the temporal sequencing of the events $\mathrm{CS}_{1}, \mathrm{CS}_{2}$, and US can be better understood through an analysis of these four types of conditioning events. Sections 3-5 describe qualitatively the mechanisms that compose a gated-dipole opponent process and contrast them with the opponent-process model of Solomon and Corbit (1974). Sections 6-16 mathematically describe the neural circuits we have developed to carry out conditioned reinforcer learning. Sections 17 and 18 display real-time computer simulations of the several types of conditioned reinforcer learning. Sections 19-31, in Part II, use these quantitative results as a basis for providing qualitative explanations of conditioning experiments in which conditioned reinforcer learning plays a part. The gated-dipole

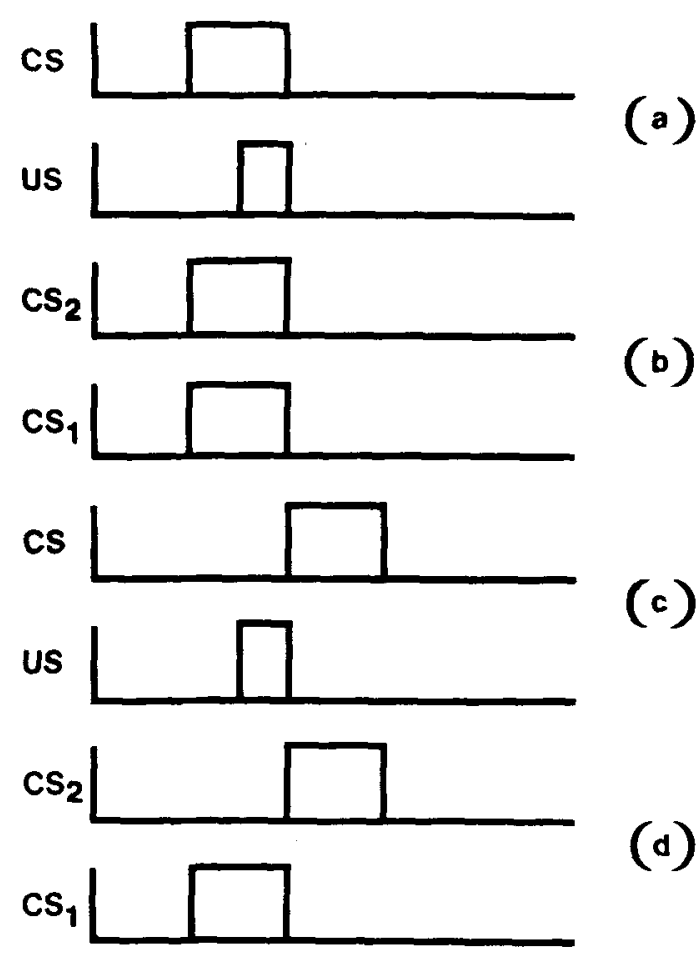

Figure 4. Some simulated combinations of conditioned stimulus (CS) and unconditioned stimulus (US) on individual trials: (a) primary excitatory conditioning; (b) secondary excitatory conditioning; (c) primary inhibitory conditioning; (d) secondary inhibitory conditioning. Because a READ circuit does not, in itself, cause blocking of simultaneously presented CSs, CS onset times in (b) were chosen to be synchronous. When blocking mechanisms are added, as in Part II, staggered $\mathrm{CS}_{1}-\mathrm{CS}_{2}$ onset times are necessary.

mechanisms described herein characterize only one of several types of model circuits that compose the total neural architecture we use to explain these data about conditioning. Mechanisms of attention, expectation, orienting, sensory and cognitive chunking, motor learning, and sensory-motor planning are no less important than gateddipole mechanisms. Mathematical analyses and extensive computer simulations of these other types of mechanisms have been reported elsewhere (Bullock \& Grossberg, in press; Carpenter \& Grossberg, 1987, in press a, in press b; Cohen \& Grossberg, 1986, 1987; Grossberg, 1987a; Grossberg \& Kuperstein, 1986; Grossberg \& Levine, in press).

\section{The READ Circuit: A Synthesis of Opponent Processing and Associative Learning Mechanisms}

A gated-dipole opponent process is a minimal neural network that is capable of generating a sustained but habituative on-response to onset of a cue, as well as a transient off-response, or antagonistic rebound, to offset of the cue (Figure 5). Properties of the on-response are used to explain excitatory conditioning, whereas properties of the off-response are used to explain inhibitory conditioning. 


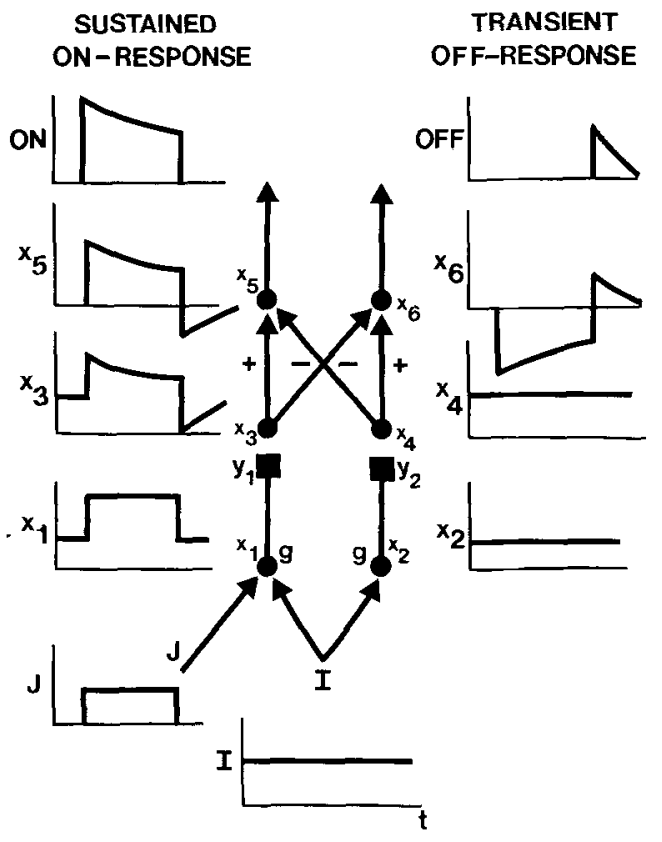

Figure 5. Example of a feedforward gated dipole. A sustained habituating on-response (top left) and a transient off-rebound (top right) are elicited in response to onset and offset, respectively, of a phasic input, J (bottom left), when tonic arousal, I (bottom center), and opponent processing (diagonal pathways) supplement the slow gating actions (square synapses). See text for details.

In order to explain secondary conditioning, a gateddipole circuit must also contain internal feedback pathways (Grossberg, 1972b, 1982a). Then a single CS, can engage in two types of events: The $\mathrm{CS}_{1}$ can learn to become an excitatory conditioned reinforcer by being paired with a subsequent US. After conditioning occurs, offset of $\mathrm{CS}_{1}$ can generate an antagonistic rebound that can condition a subsequent $\mathrm{CS}_{2}$ to become an inhibitory secondary reinforcer. When a neural network contains internal feedback pathways, it is said to be recurrent.

In order to explain these several types of conditioned reinforcer learning, such a recurrent gated dipole must be joined to a mechanism of associative learning. Thus the total circuit analyzed here is called a recurrent associative gated dipole (READ) circuit.

A number of design constraints must be satisfied simultaneously by a READ circuit. The opponent processing laws and the associative learning laws must fit together in such a way that all the desired properties of conditioned reinforcer learning obtain with a single choice of parameters. Moreover, the range of parameters for which this is true must be robust. A READ circuit contains processes that fluctuate on three different time scales-a fast activation time scale, a slower habituation time scale, and a yet slower conditioning time scale. As noted above, these processes are linked together by nonlinear feedback interactions, due to the recurrent anatomy of the circuit. The design of nonlinear feedback circuits that possess three distinct time scales is a difficult task in any scien- tific discipline. That a READ circuit forms only one of the several circuits that are engaged during conditioning, and that all of these circuits interact via nonlinear feedback signals, highlights the difficulty of building a rigorous real-time conditioning theory. On the other hand, many additional design constraints become evident when one actually attempts to build such a theory. The simultaneous satisfaction of these several stringent design requirements has led us to some neurophysiologically testable predictions about how associative learning is regulated by gated-dipole opponent processing.

\section{Qualitative Properties of a Gated Dipole}

Four main ingredients go into the design of a gated dipole: slowly habituating and recovering chemical transmitters; opponent, or competitive, interactions between an on-channel and an off-channel; phasic inputs, such as a CS or US, that perturb the on-channel or the off-channel through time; and a sustained, or tonic, arousal input that equally perturbs both channels, thereby setting the sensitivity of dipole outputs to phasic input fluctuations and providing the energy to generate an antagonistic rebound in response to offset of an input.

Figure 5 describes the simplest type of feedforward, or nonrecurrent, gated dipole. Figure 5 also schematizes how a gated dipole can generate a sustained, but habituative, on-response to input onset, and a transient offresponse, or antagonistic rebound, to input offset. These reactions can be qualitatively explained as follows. (See Grossberg, 1972b, 1984b, 1987a, for quantitative mathematical analyses.)

A. Transmitter gating. Signals in both the on-channel and the off-channel are multiplied, or gated, by a chemical transmitter (square synapses) before the gated signals are further transformed by opponent processing. Each transmitter $y(t)$ multiplies its input signal $S(t)$ to form such a gated output signal $T(t)$; that is,

$$
T=S y \text {. }
$$

B. Slow transmitter habituation and recovery. The transmitter $y$ habituates and recovers according to the law (Grossberg, 1968, 1972b):

$$
\frac{d}{d t} y=B(1-y)-C S y,
$$

where $B$ and $C$ are positive constants. In Equation 2, the notation $\frac{d}{d t} y$ denotes the net rate of change of $y$. Term $B(1-y)$ says that transmitter $y$ recovers at a rate $B$ until it reaches the target level 1 . Term -CSy says that transmitter $y$ habituates at a rate proportional to its gating action in Equation 1.

Many refinements of the laws given by Equations 1 and 2 have been described, including equations for transmitter mobilization, post-tetanic potentiation, enzymatic activation, and autoreceptive competition (Carpenter \& Grossberg, 1981; Grossberg, 1969b, 1987a, 1987b). These refinements, albeit important for some purposes, 
do not play a major role in explaining qualitative properties of conditioned reinforcer learning.

C. Overshoot and undershoot. A critical property for explaining conditioned reinforcer data is that the input signal $S$ can fluctuate more quickly than the transmitter $y$ can react. This property leads to an overshoot in the output $T$ in response to onset of the input $S$ and an undershoot in $T$ in response to offset of $S$ (Figure 6). To see how this happens, note that in response to a constant input of size $S$, Equation 2 implies that the transmitter $y$ approaches the equilibrium value

$$
y=\frac{B}{B+C S} \text {. }
$$

In other words, larger signals $S$ cause more transmitter habituation. On the other hand, the output signal that is generated by an input $S$ does not equal $y$. The output signal is equal to $T=S y$, due to Equation 1 .

Figure 6 describes how the output $T$ reacts to changes in the size of the input $S$. A rapid increase in $S$ from $S_{0}$ to $S_{1}$ elicits a slow decrease in $y$, due to Equation 3. Multiplication of the graphs of $S(t)$ and $y(t)$ shows that a rapid increase in $S$ generates a rapid increase in $T$, followed by a slow decrease, or habituation, of $T$ to an intermediate level. In a similar way, a rapid decrease in $S$ from $S_{1}$ to $S_{0}$ generates a rapid decrease in $T$, followed by a slow increase, or habituation, to an intermediate level.
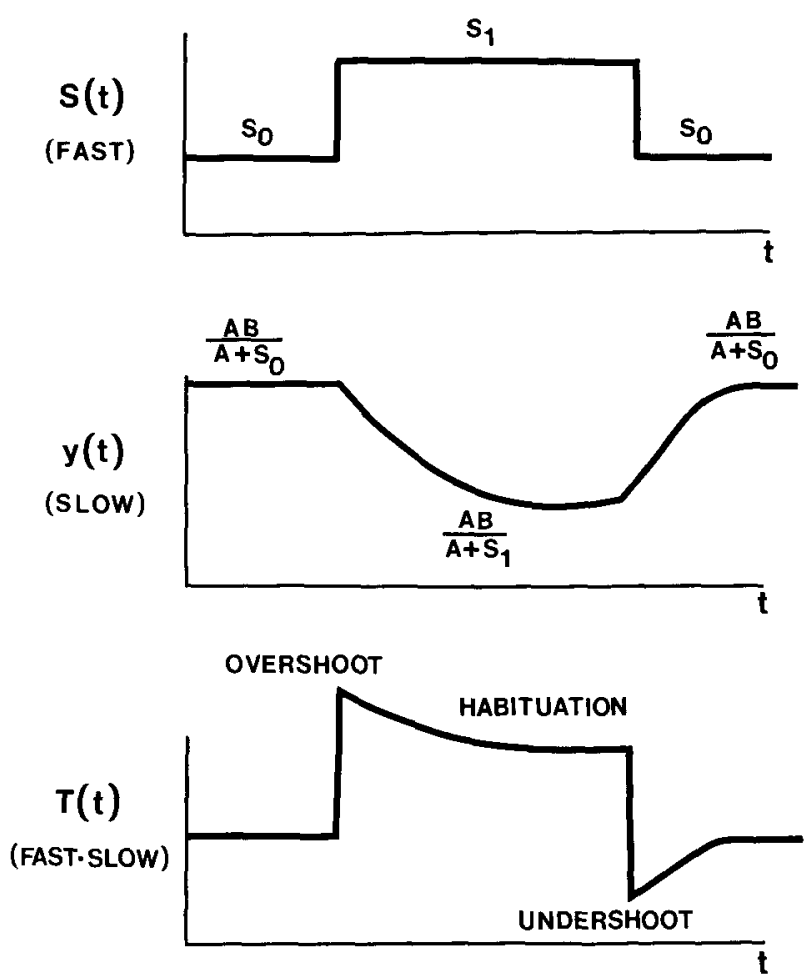

Figure 6. Reaction of output signal $T$ and transmitter gate $y$ to changes in input $S$. The output $T$ is the product of a fast process, $S$, and a slow process, $y$. Overshoots and undershoots in $T$ are caused by y's slow habituation to fast changes in $S$.
In all, rapid increases and decreases in the input $S$ generate overshoots and undershoots in the output $T$ due to the slow rate of reaction, or habituation, of the transmitter. These habituative reactions are fundamental to many basic properties of gated dipoles and, by extension, of conditioned reinforcer learning.

D. Tonically aroused transmitter gates in opponent processes. We can now explain the properties depicted in Figure 5. In such an opponent process, a phasic input $(J)$ can elicit a sustained on-response, whereas offset of the input can elicit a transient off-rebound, or temporal contrast effect. These properties are explained as follows.

The left-hand series of stages in Figure 5 represents the on-channel, and the right-hand series of stages represents the off-channel. Both channels receive an equal arousal input, denoted by $I$, that is constant through time. The arousal input provides the tonic internal activity that triggers the antagonistic rebound that occurs after the on-input shuts off. The on-input, denoted by $J$, is delivered only to the on-channel. Input $J$ is switched from zero to a positive level and held at that level long enough for transmitter habituation to occur. Then $J$ is shut off.

Inputs $I$ and $J$ are added by the activity (or potential) $x_{1}(t)$. Activity $x_{1}(t)$ responds quickly to input fluctuations, relative to the reaction rate of the network's slow transmitter gates. The graph of $x_{1}(t)$ has the same form as the top graph in Figure 6: a rapid switch from a lower positive activity to a higher positive activity, followed by a rapid return to the lower level. The activity $x_{1}(t)$ generates an output signal $g\left(x_{1}(t)\right)$ in its pathway that again has the form of a double switch between two positive values. The output signal $g\left(x_{1}(t)\right)$ is gated by a slow transmitter $y_{1}(t)$ that accumulates and habituates within the square synapse in the on-channel. Figure 6 describes the effect of this slow gate on the input to the next stage. Consequently, activity $x_{3}(t)$ follows an overshoot-habituation-undershoot-habituation sequence through time. Then $x_{3}(t)$ relays an output signal of the same form to $x_{5}(t)$. Activity $x_{5}(t)$ also receives an inhibitory signal from $x_{4}(t)$. To determine what happens next, we consider the dynamics of the off-channel.

The off-channel receives only the constant tonic input 1. Hence $x_{2}(t)$ and the slow gate $y_{2}(t)$ in the off-channel square synapses are constant through time. The activity $x_{4}(t)$ is therefore also constant through time. For definiteness, we make the simplest assumption that corresponding stages in the on-channel and the off-channel possess the same parameters. Since the arousal input $I$ to both channels is also equal, the size of $x_{4}$ equals the baseline activity level of $x_{3}(t)$. This is not always true, but its violation is easy to analyze after the symmetric case is understood (Grossberg, 1984b).

We can now determine the reactions of activity $x_{5}(t)$ through time. Since the signals from $x_{3}(t)$ and $x_{4}(t)$ subtract before perturbing $x_{5}(t)$, and their baseline activities are the same, the baseline activity of $x_{5}(t)$ equals zero. Activity $x_{5}(t)$ thus overshoots and undershoots a zero baseline when the input $J$ is turned on and off. By contrast, activity $x_{6}(t)$ responds in an opposite way from $x_{5}(t)$ be- 
cause $x_{3}$ excites $x_{5}$ and inhibits $x_{6}$, whereas $x_{4}$ inhibits $x_{5}$ and excites $x_{6}$.

The final assumption is that the output signals caused by activities $x_{5}(t)$ and $x_{6}(t)$ are rectified: outputs are generated only if these activities exceed a nonnegative threshold. As a result, the on-channel generates a sustained output signal while the input $J$ is on. This output signal habituates as the gate $y_{1}(t)$ slowly equilibrates to the input. By contrast, the off-channel generates a transient offresponse, or antagonistic rebound, after the input $J$ shuts off.

\section{Comparison With the Solomon and Corbit Opponent-Process Model}

The antagonistic rebound in the off-channel of a gated dipole is energized by an undershoot of the dipole's onactivity function $x_{3}$ (Figure 5). In a gated dipole, such an undershoot is due to habituation of the transmitter gate within the on-channel. Overshoots and undershoots have also been hypothesized to exist in alternative models of opponent processing, but the properties have not been traced to the action of a slowly habituating transmitter gate. For example, Solomon and Corbit (1974) and Solomon $(1980,1982)$ described a model of opponent processing in which overshoots and undershoots occur. These authors ascribed the overshoots and undershoots to the subtraction of two opponent processes that both evolve according to similar time scales (Figure 7). Neither process, in itself, undergoes an overshoot or an undershoot. Instead, overshoots and undershoots are derived from the assumption that the off-process begins to build up only after the on-process is initiated. The model assumes, in addition, that "the second component, the $b$ process, is aroused via the arousal of $a$ " (Solomon \& Corbit, 1974, p. 126). Neither assumption is made in a gateddipole opponent process, wherein the slow habituation of the transmitter gate within the on-channel generates an overshoot and an undershoot within that channel. Consequently, in a gated dipole, opponent processing per se between the on-channel and the off-channel generates the antagonistic rebound within the off-channel without necessitating the hypothesis that on-channel activation triggers a delayed off-channel activation.

Solomon and Corbit's (1974) opponent process is not defined by a dynamic model such as the gated-dipole architecture. Thus their model does not explain why the maximum size of the $a$ process should sometimes, but not always, exceed the maximum size of the $b$ process, or why the $b$ process is delayed in time relative to the $a$ process by just the right amount to produce an overshoot and an undershoot. The hypothesis that slowly habituating, tonically aroused transmitter gates exist in an opponent anatomy provides simple answers to all of these questions, and implies other properties that enable the gateddipole model to explain data about conditioned reinforcer learning.
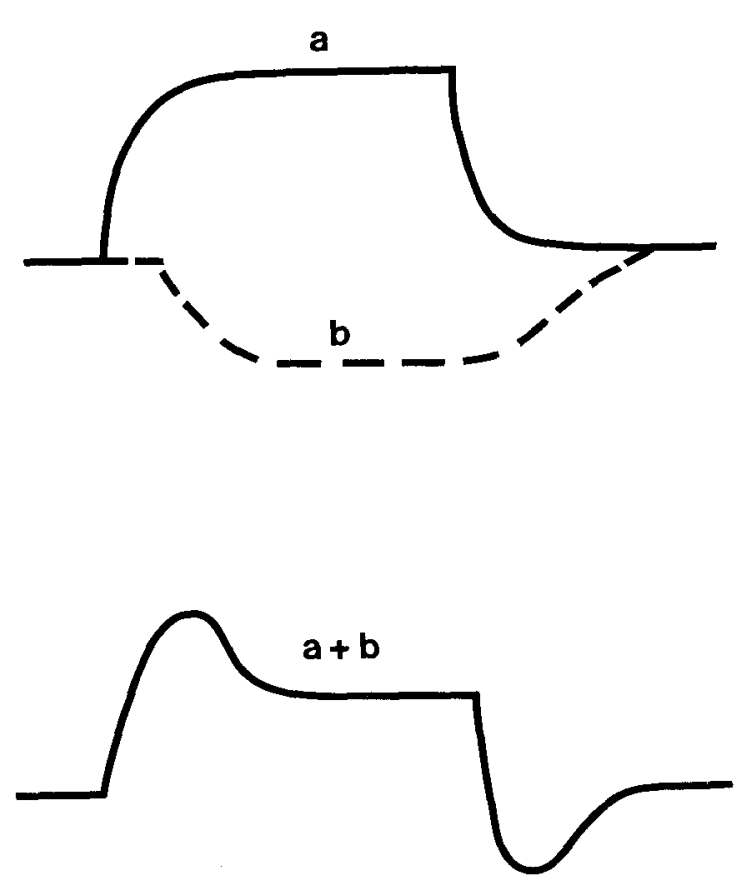

Figure 7. In the opponent-process model of Solomon (1982), overshoots and undershoots are caused by an excitatory process (a) and an inhibitory process (b), which both change at a similar rate such that (b) lags behind (a) and neither (a) nor (b) separately exhibits overshoots or undershoots.

\section{Laws for a READ Circuit}

Dynamic equations for a READ circuit are described and explained in this section. Our analysis has revealed that several variations on the same basic network design have the properties that we desire. Which variation may exist in particular species is testable by neurophysiological and anatomical techniques. The simplest network variation is depicted in Figure 3. This circuit will be defined first. Then the functional significance of its equations will be explained. After that, the equations corresponding to the other circuits will be explained.

As in the nonrecurrent gated dipole described in Section 4 , the variables $x_{i}$ describe cell potentials, or activations, and the variables $y_{i}$ describe slowly habituating transmitter gates. In addition, the variables $z_{k l}$ describe long-term memory (LTM) traces, or associative weights, that exist at the ends of the pathways from the sensory representations of CS and US cues to the on-channel and the off-channel of the gated dipole. The equations for the READ I circuit are as follows:

\section{READ I EQUATIONS}

Arousal + US + Feedback On-Activation

$$
\frac{d}{d t} x_{1}=-A x_{1}+I+J+f\left(x_{7}\right)
$$


Arousal + Feedback Off-Activation

$$
\frac{d}{d t} x_{2}=-A x_{2}+I+f\left(x_{8}\right)
$$

\section{On-Transmitter}

$$
\frac{d}{d t} y_{1}=B\left(1-y_{1}\right)-C g\left(x_{1}\right) y_{1}
$$

\section{Off-Transmitter}

$$
\frac{d}{d t} y_{2}=B\left(1-y_{2}\right)-C g\left(x_{2}\right) y_{2}
$$

\section{Gated On-Activation}

$$
\frac{d}{d t} x_{3}=-A x_{3}+D g\left(x_{1}\right) y_{1}
$$

Gated Off-Activation

$$
\frac{d}{d t} x_{4}=-A x_{4}+D g\left(x_{2}\right) y_{2}
$$

Normalized Opponent On-Activation

$$
\frac{d}{d t} x_{5}=-A x_{5}+\left(E-x_{5}\right) x_{3}-\left(x_{5}+F\right) x_{4}
$$

\section{Normalized Opponent Off-Activation}

$$
\frac{d}{d t} x_{6}=-A x_{6}+\left(E-x_{6}\right) x_{4}-\left(x_{6}+F\right) x_{3}
$$

\section{On-Activation by CS Inputs}

$$
\frac{d}{d t} x_{7}=-A x_{7}+G\left[x_{5}\right]^{+}+L \sum_{k} S_{k} z_{k 7},
$$

where $[w]^{+}=\max (w, 0)$.

Off-Activation by CS Inputs

$$
\frac{d}{d t} x_{8}=-A x_{8}+G\left[x_{6}\right]^{+}+H \sum_{k} S_{k} z_{k 8}
$$

On-Conditioned Reinforcer Learning

$$
\frac{d}{d t} z_{k 7}=S_{k}\left(-K z_{k 7}+L\left[x_{5}\right]^{+}\right)
$$

Off-Conditioned Reinforcer Learning

$$
\frac{d}{d t} z_{k 8}=S_{k}\left(-K z_{k 8}+L\left[x_{6}\right]^{+}\right)
$$

\section{On-Output Signal}

$$
O_{1}=\left[x_{5}\right]^{+}
$$

Off-Output Signal

$$
O_{2}=\left[x_{6}\right]^{+}
$$

7. Tonic Arousal, Phasic US Input, and Feedback Signaling

In Equations 4 and 5, term $I$ denotes the tonically active arousal level that sets the baseline sensitivity of the READ circuit and energizes its antagonistic rebounds. Term $J$ in Equation 4 denotes the US input. This US input corresponds to a primary US, not to a conditioned reinforcer that acquires US properties through conditioning.

Term $f\left(x_{7}\right)$ in Equation 4 describes the nonnegative signal that converts the on-channel into a positive feedback loop. In a similar fashion, term $f\left(x_{8}\right)$ in Equation 5 describes the nonnegative signal that converts the offchannel into a positive feedback loop. Terms $-A x_{1}$ and $-A x_{2}$ in Equations 4 and 5, respectively, describe the passive decay terms whereby the potentials $x_{1}$ and $x_{2}$ return to the equilibrium value 0 . The equations governing all the potentials $x_{i}$ contain such passive decay terms $-A x_{i}$. For simplicity, the same parameter $A$ was chosen in all of these equations.

\section{Gating Fast Signals with Slowly Habituating Transmitters}

Equations 6 and 7 describe the dynamics of the habituating transmitters $y_{1}$ and $y_{2}$ in the on-channel and the offchannel, respectively. These equations are the same as Equation 2. In Equation 6, the nonnegative input signal $S=g\left(x_{1}\right)$, whereas in Equation 7, the nonnegative input signal $S=g\left(x_{2}\right)$.

Equation 8 describes the effect of the gated on-channel signal $D g\left(x_{1}\right) y_{1}$ on the next on-potential $x_{3}$. Potential $x_{3}$ averages these gated signals through time at rate $-A$. In a similar fashion, Equation 9 describes the effect of the gated off-channel signal $D g\left(x_{2}\right) y_{2}$ on the next off-potential $x_{4}$.

\section{Normalized Opponent Interactions}

Equations 10 and 11 describe the effects of opponent, or competitive, signals from $x_{3}$ and $x_{4}$ on the next onpotential $x_{5}$ and off-potential $x_{6}$. Equation 10 is a membrane, or shunting, equation of the form

$C \frac{d V}{d t}=\left(V^{p}-V\right) g^{p}+\left(V^{+}-V\right) g^{+}+\left(V^{-}-V\right) g^{-}$,

where $C$ is a capacitance (scaled to equal 1 for convenience); $V^{p}, V^{+}$, and $V^{-}$are saturation potentials; $g^{p}$, $g^{+}$, and $g^{-}$are conductances; and $V$ is a variable potential. See Grossberg (1982c, 1987a, 1987b) and Grossberg and Kuperstein (1986) for many applications of this equation. The additive equations $4,5,7$, and 8 may be interpreted as approximations to Equation 18 whose inputs are not large enough to drive their potentials close to their saturation potentials $V^{+}$and $V^{-}$.

The crucial properties of a shunting equation can be appreciated by studying its equilibrium values. At equilibrium, $\frac{d}{d t} x_{5}=0$. Then Equation 10 implies 


$$
x_{5}=\frac{(E+F)\left(x_{3}+x_{4}\right)}{A+x_{3}+x_{4}}\left[\frac{x_{3}}{x_{3}+x_{4}}-\frac{F}{E+F}\right]
$$

(Grossberg, 1970, 1976b, 1983). In Equation 19, if $x_{3}+x_{4} \gg A$, then term $(E+F)\left(x_{3}+x_{4}\right)\left(A+x_{3}+x_{4}\right)^{-1}$ is approximately constant. Then Equation 19 implies that $x_{5}$ is sensitive to the ratio $x_{3}\left(x_{3}+x_{4}\right)^{-1}$ relative to the adaptation level $F(E+F)^{-1}$. Thus Equation 10 automatically regulates the overall operating range of the circuit. In addition, $x_{5}>0$ in Equation 19 only if

$$
\frac{x_{3}}{x_{3}+x_{4}}>\frac{F}{E+F} \text {. }
$$

Since the output signal due to $x_{5}$ is $\left[x_{5}\right]^{+}$in Equations 12, 14 , and 16 , all subsequent processing by the on-channel is controlled by whether or not the relative size of $x_{3}$ to $x_{4}$ enables ratio $x_{3}\left(x_{3}+x_{4}\right)^{-1}$ to exceed the constant adaptation level $F(E+F)^{-1}$. Thus Equation 10 evaluates whether the total balance of all factors influencing the network favors the on-channel over the off-channel enough to cause the inequality given in Equation 20 to hold.

In the circuit depicted in Figure 3, we chose $E=F$ in Equation 10. Then Equation 19 may be more simply written as

$$
x_{5}=\frac{E\left(x_{3}-x_{4}\right)}{A+x_{3}+x_{4}} .
$$

In this special case, $x_{5}>0$ only if $x_{3}>x_{4}$. Thus $\left[x_{5}\right]^{+}>0$ only if the balance of all network factors favors the on-channel over the off-channel. In addition, the denominator $A+x_{3}+x_{4}$ in Equation 21 ensures that $x_{5}$, and likewise $x_{6}$, computes a ratio scale, in addition to an opponent scale, from $x_{3}$ and $x_{4}$.

Equation 11 for $x_{6}$ is the same as Equation 10 for $x_{5}$ with the opponent input terms $x_{3}$ and $x_{4}$ reversed. Thus at equilibrium, when $E=F$ in Equation 11,

$$
x_{6}=\frac{E\left(x_{4}-x_{3}\right)}{A+x_{3}+x_{4}} .
$$

By Equations 21 and 22,

$$
\operatorname{sgn}\left(x_{5}\right)=-\operatorname{sgn}\left(x_{6}\right)
$$

where

$$
\operatorname{sgn}(w)=\left\{\begin{array}{cl}
+1 & \text { if } w>0 \\
0 & \text { if } w=0 \\
-1 & \text { if } w<0
\end{array}\right.
$$

In summary, if $E=F$, then $x_{5}$ and $x_{6}$ compute a normalized opponent process.

\section{Positive and Negative Conditioned Reinforcer Inputs: Total Context Versus Individual Cue}

Equation 12 registers the normalized opponent signal $\left[x_{5}\right]^{+}$from the on-channel, as well as a sum $L \Sigma_{k} S_{k} z_{k 7}$ of signals due to all CSs and conditioned USs. Term $S_{k}$ is the output signal from the $k$ th sensory representation. This signal is multiplied, or gated, by the LTM trace $z_{k 7}$ at the end of the pathway from the $k$ th sensory representation to the on-channel of the READ circuit. The sum $L \Sigma_{k} S_{k} z_{k 7}$ is called the total positive conditioned reinforcer signal.

In a similar fashion, Equation 13 registers the normalized opponent signal $\left[x_{6}\right]^{+}$from the off-channel, as well as the total negative conditioned reinforcer signal $L \sum_{k} S_{k} z_{k 8}$. Thus the output signal $S_{k}$ from the $k$ th sensory representation is gated by an LTM trace $z_{k 7}$ abutting the READ on-channel and an LTM trace $z_{k 8}$ abutting the READ off-channel. Due to the opponent organization of the READ circuit, the $k$ th sensory representation is a positive conditioned reinforcer if

$$
z_{k 7}>z_{k 8}
$$

and a negative conditioned reinforcer if

$$
z_{k 7}<z_{k 8} .
$$

These inequalities determine the conditioned reinforcer properties of a single sensory event. In general, many active sensory events may simultaneously input to the READ circuit. Then the total behavioral environment behaves like a positive conditioned reinforcer context if

$$
\sum_{k} S_{k} z_{k 7}>\sum_{k} S_{k} z_{k 8}
$$

and like a negative conditioned reinforcer context if

$$
\sum_{k} S_{k} z_{k 7}<\sum_{k} S_{k} z_{k 8}
$$

(Grossberg, 1972a, 1972b). Clearly, a positive conditioned reinforcer context can obtain even if it contains active negative reinforcers, and vice versa.

\section{Context-Dependent Adaptation Level and Associative Averaging}

The total positive and negative conditioned reinforcer signals interact within a gated-dipole circuit to cause context-dependent, and hence learning-dependent, shifts in the circuit's adaptation level (Grossberg, 1972b, 1987a). The adaptation level is the baseline level of tonic activation that is maintained across both the on-channel and the off-channel of the circuit during a time interval that is long enough to modulate the circuit's habituation, rebound, or conditioning properties. Changes in the total configuration of conditioned reinforcing cues, including contextual cues, can dramatically alter the dynamics of a READ circuit by changing its adaptation level. This fact will be critical in explaining the data summarized in Part II. The main factors that control the circuit's adaptation level are now summarized.

In the absence of any inputs to the gated dipole, both the on-channel and the off-channel become equally active; thus $x_{1}=x_{2}, y_{1}=y_{2}, x_{3}=x_{4}, x_{5}=x_{6}$, and $x_{7}=x_{8}$. In the READ I circuit, the choice $E=F$ implies, in addition, that $x_{5}=x_{6}=0$ by Equations 21 and 22, and thus that $x_{7}=x_{8}=0$, by Equations 12 and 13. Consequently, in the no-input case, the adaptation level equals the tonic arousal level $I$ that is defined by Equations 4 and 5 . 
In contrast, when conditioned reinforcers are active, the terms $f\left(x_{7}\right)$ in Equation 4 and $f\left(x_{8}\right)$ in Equation 5 can cause an increase in the adaptation level. To understand this property more precisely, consider the following facts. The potentials $x_{7}$ and $x_{8}$ react quickly to their input signals. Hence during a time interval when the conditioned reinforcer signals $S_{k}$ are maintained, $x_{7}$ and $x_{8}$ can achieve an approximate equilibrium with respect to these signals. Then $\frac{d}{d t} x_{7} \cong 0$ and $\frac{d}{d t} x_{8} \cong 0$ in Equations 12 and 13 , respectively, whence

$$
x_{7} \cong \frac{G}{A}\left[x_{5}\right]^{+}+\frac{L}{A} \sum_{k} S_{k} z_{k 7}
$$

and

$$
x_{8} \cong \frac{G}{A}\left[x_{6}\right]^{+}+\frac{L}{A} \sum_{k} S_{k} z_{k 8}
$$

In Equations 4 and 5, we chose $f\left(x_{7}\right)=M x_{7}$ and $f\left(x_{8}\right)=$ $M x_{8}$ in our computer simulations. Hence by Equations 29 and 30 ,

$$
\frac{d}{d t} x_{1} \cong-A x_{1}+I+J+\frac{M G}{A}\left[x_{5}\right]^{+}+\frac{M L}{A} \sum_{k} S_{k} z_{k 7}
$$

and

$$
\frac{d}{d t} x_{2} \cong-A x_{1}+I+\frac{M G}{A}\left[x_{6}\right]^{+}+\frac{M L}{A} \sum_{k} S_{k} z_{k 8} .
$$

Guided by Equations 31 and 32, we define the circuit's adaptation level $\hat{I}$ by the minimum of $I+J+$ $\frac{M L}{A} \sum_{k} S_{k} z_{k 7}$ in Equation 31 and of $I+\frac{M L}{A} \sum_{k} S_{k} z_{k 8}$ in Equation 32; that is,

$$
\hat{I}=\min \left[I+J+\frac{M L}{A} \sum_{k} S_{k} z_{k 7}, I+\frac{M L}{A} \sum_{k} S_{k} z_{k 8}\right]
$$

(Figure 8). In other words, $\hat{l}$ describes the tonic baseline due to the totality of internally generated tonic arousal signals and externally generated primary and secondary reinforcer signals. Variables $x_{1}$ and $x_{2}$ in Equations 4 and 5 activate $x_{3}$ and $x_{4}$, which compete to generate $x_{5}$ and $x_{6}$ before these net activations regulate the READ circuit's antagonistic rebounds and conditioning signals. Thus the net input signal that determines whether rebounds or conditioning will occur is the difference

$$
\Delta=J+\frac{M L}{A} \sum_{k} S_{k}\left(z_{k 7}-z_{k 8}\right)
$$

of the total arousal and reinforcing signals $I+J+$ $\frac{M L}{A} \sum_{k} S_{k} z_{k 7}$ and $I+\frac{M L}{A} \sum_{k} S_{K} z_{k 8}$ that define the adaptation level. If all primary reinforcers and conditioned reinforcers balance out so that $\Delta=0$, then their only effect on the gated dipole is to cause a shift in adaptation level. No new conditioning occurs under these circumstances because equal total inputs to $x_{1}$ and $x_{2}$ cause $x_{5}=x_{6}=0$ after the transmitter gates $y_{1}$ and $y_{2}$ habituate to these equal total inputs. If $\hat{l}$ is very large but $\Delta$ is very small

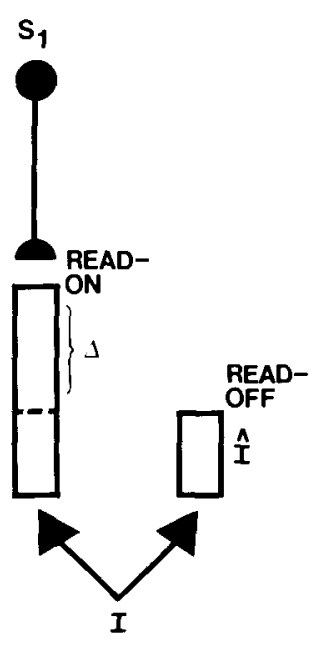

(a)

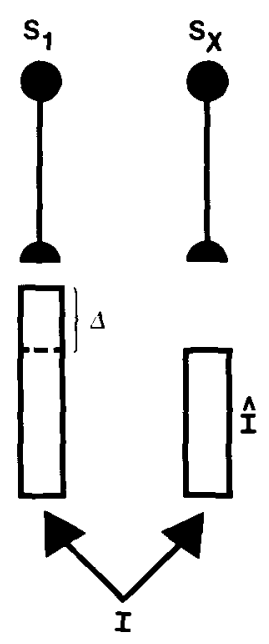

(b)
Figure 8. (a) If the total input to the on-channel of the READ circuit is large, whereas the total input to the off-channel is small, then $\hat{I}$ is small and $\Delta$ is large and positive. (b) If the total inputs to both channels are large, then $\bar{I}$ is large and $\Delta$ is small.

(Figure 8b), then any conditioning that does occur is weak, because $x_{5}$ and $x_{6}$ in Equations 21 and 22 would both be close to zero due to the normalization property. If $\Delta>0$, then conditioning of positive conditioned reinforcers occurs, due to Equations 21 and 14 . If $\Delta<0$, then conditioning of negative conditioned reinforcers occurs, due to Equations 22 and 15 . Thus a contextual cue that is a potent positive reinforcer can interfere with conditioning of a discrete $\mathrm{CS}$ as a negative reinforcer, and vice versa.

An important constraint on the terms $\hat{I}$ and $\Delta$ (see Part II for details) follows from the property that the total STM activation that reads out the signals $S_{k}$ also tends to be normalized, or conserved, at each time (Grossberg, 1972a, 1975, 1982c). This normalization property explicates the concept of a limited-capacity STM, or working memory, that is operative during Pavlovian conditioning. In its simplest form, the normalization property may be realized by the constraint that

$$
\sum_{k} S_{k}=S \equiv \text { constant. }
$$

It then follows from Equations 33 and 34 that both $\hat{I}$ and $\Delta$ are determined by a type of associative averaging, rather than by summation. In particular, term

$$
\sum_{k} S_{k}\left(z_{k 7}-z_{k 8}\right)
$$

in Equation 34 is a weighted average, with weights equal to the net LTM strengths $z_{k 7}-z_{k 8}$ of the signals $S_{k}$. By Equation 36, any mechanism that increases a signal $S_{k}$ that generates a net positive conditioned reinforcer input $\left(z_{k 7}>z_{k 8}\right)$ to the gated dipole a fortiori weakens the total net negative conditioned reinforcer input to the gated 
dipole. On the other hand, such an increase may or may not increase the total net positive conditioned reinforcer input to the dipole, because the increase in one positive input may be balanced by a decrease in a different positive net input. Thus there exists an asymmetry in the net effect that an attention shift among the sensory representations may cause on the overall performance of a READ circuit.

\section{Associative Learning: Learned LTM Increases or Decreases Gated by CS Read-Out}

Equation 14 describes the associative learning law whereby the positive conditioned reinforcer LTM trace is trained. This associative learning law was introduced into the associative learning literature by Grossberg (1969a) and has played a central role in the development of neural architectures in a variety of applications (Carpenter \& Grossberg, 1987, in press a; Cohen \& Grossberg, 1987; Grossberg, 1982c, 1987a, 1987b; Grossberg \& Levine, in press; Grossberg \& Stone, 1986b). Recently, direct neurophysiological evidence for this associative learning law has been reported (Levy, 1985; Levy, Brassel, \& Moore, 1983; Levy \& Desmond, 1985; Rauschecker \& Singer, 1979; Singer, 1983). In Equation 14 , the signal $S_{k}$ from the $k$ th sensory representation turns learning of the LTM trace $z_{k 7}$ on and off. When $S_{k}=0$, learning turns off because $\frac{d}{d t} z_{k 7}=0$. When $S_{k}>0$, learning turns on. Thus activation of a sensory representation both reads out a conditioned reinforcer signal, via term $S_{k} z_{k 7}$ in Equation 12, and reads in new learned information, via Equation 14. When $S_{k}>0$, the LTM trace performs a time-average, at rate $-K S_{k}$, of the learning signal $L S_{k}\left[x_{5}\right]^{+}$. As a result, the LTM trace $z_{k 7}$ attempts to track the normalized opponent signal $\left[x_{5}\right]^{+}$ through time. In particular, during a time interval $t_{0} \leq t \leq t$, when $S_{k}(t)$ equals a positive constant $S_{k}$, Equation 14 may be integrated to yield

$$
z_{k 7}(t)=z_{k 7}\left(t_{0}\right) e^{-K S_{k}\left(t-t_{0}\right)}+L \int_{t_{0}}^{t}\left[x_{5}(v)\right]^{+} e^{-K S_{k}(t-v)} d v
$$

In other words, $z_{k 7}$ performs a time-average of $\left[x_{5}\right]^{+}$at a rate proportional to $S_{k}$. Due to this property, $z_{k 7}$ can either decrease (when $\left[x_{5}\right]^{+}$becomes small for a time) or increase (when $\left[x_{5}\right]^{+}$becomes large for a time). This learning property is critical in our work.

\section{Dissociation of LTM Read-In and Read-Out: A Possible Role for Dendritic Spines}

A key property of the READ circuit may be understood by comparing Equations 12,14 , and 16 . This property is the basis for the opponent extinction property (Section 24) that is used to explain extinction of a conditioned excitor (Section 27) and nonextinction of a conditioned inhibitor (Section 28).

By Equation 12, prior conditioned reinforcer learning is read out via term $L \sum_{k} S_{k} z_{k 7}$ to activate the potential $x_{7}$. In contrast, $x_{7}$ does not appear in the learning equation 14 .

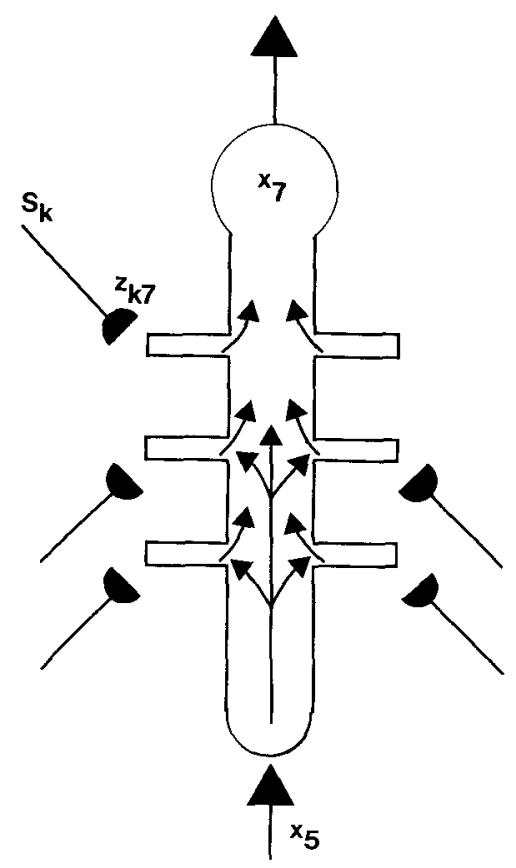

Figure 9. A possible microarchitecture for dissociation of longterm memory (LTM) read-in and read-out. Individual LTM-gated sensory signals, $S_{k} z_{k} 7$, are read out into local potentials that are summed by the total cell-body potential, $x_{7}$, without significantly influencing each other's learned read-in. In contrast, the input sig$n a l, x_{5}$, triggers a massive global cell activation that drives learned read-in at all active LTM traces abutting the cell surface. Signal $x_{5}$ also activates the cell-body potential $x_{7}$.

Instead, $\left[x_{5}\right]^{+}$appears in Equation 14. Thus LTM readout and LTM read-in are dissociated in Equations 12 and 14. In addition, the term $\left[x_{5}\right]^{+}$, which is read into LTM by Equation 14, is the on-channel output signal, as in Equation 16. Thus the READ circuit embodies the intuition that the signals that drive learning and elicit outputs to other circuits are the resultant of all the decision-making processes that take place within the circuit. In particular, $\left[x_{5}\right]^{+}$is a normalized opponent signal, whereas $x_{7}$ is not.

How can such a dissociation between LTM read-in and LTM read-out be physiologically implemented? The scheme we apply was introduced by Grossberg (1975; reprinted in Grossberg, 1982c) for this purpose. Figure 9 schematizes this mechanism. Grossberg (1975) interpreted this formal mechanism in terms of the dynamics of large pyramidal cells which, in his application, were interpreted to occur in the hippocampus. These cells possess a large and complex dendritic tree whose activations and inhibitions generate local potentials that flow into, and are averaged by, the cell body. Due to the geometry and electrical properties of such a dendritic tree, an input that activates a particular dendritic branch may not be influenced by inputs that activate different dendritic branches. In order to maximize the functional independence of each conditionable input channel, it was assumed that the conditionable 
signals reach dendritic spines. Here they produce local potentials that propagate to the cell body where they influence axonal firing. We assume that the resistance in spines are such that it is much harder for a signal to pass between spines than from a spine to the cell body. ... By contrast, ... feedback ... causes a spike potential, or similar global potential change, throughout the dendritic column. This spike invades all the spines in its path and is sufficiently strong to induce transmitter level changes in active $S \rightarrow A$ [conditionable] channels. Thus a mechanism using dendritic spines and dendritic spike generators (or some formally analogous mechanism) can allow $S \rightarrow A$ signals to occur without major changes in $S \rightarrow A$ synaptic transmitter levels unless feedback invades the entire dendritic apparatus. (Grossberg, 1975, Section 21, p. 320)

In Figure 9, the feedback signal that invades the entire dendritic apparatus equals $\left[x_{5}\right]^{+}$. Potential $x_{7}$ computes the cell body activation that averages $G\left[x_{5}\right]^{+}$with the total positive conditioned reinforcer input $L \sum_{k} S_{k} z_{k 7}$ that is delivered at spines distributed across the entire dendritic apparatus.

The same mechanism is used to interpret Equation 13. Here term $G\left[x_{6}\right]^{+}$is the off-channel feedback signal and term $L \Sigma_{k} S_{k} z_{k \mathrm{~g}}$ is the total negative conditioned reinforcer input.

Recent experiments have supported the hypothesis that synaptic plasticity may occur at the dendritic spines of hippocampal pyramidal cells (Lynch, 1986). In addition, the same functional properties that recommended dissociation of LTM read-in and LTM read-out during hippocampal learning also recommended its use during associative learning in mammalian neocortex (Grossberg, 1982b, 1987a). Recent computer simulations of the unitization, or chunking, of cognitive recognition codes have argued for the functional importance of this concept in other cortical systems (Cohen \& Grossberg, 1987), but direct experimental evidence relevant to this prediction seems as yet to be lacking.

\section{Decoupling the Normalization and Opponent-Processing Stages}

The READ II circuit depicted in Figure 10 is mathematically equivalent to the READ I circuit in Figure 3. The READ II circuit is included to point out that the normalization and opponent-processing transformations, which are carried out in a single step by Equations 10 and 11 , may in principle be carried out separately in two successive steps. Such a dissociation may be necessary in vivo because the inhibitory saturation point $-F$ in Equations 10 and 11 is often much smaller in absolute value than the excitatory saturation point $E$; that is, $E \gg F$. In fact, cells are known to exist in which $F$ is approximately zero (e.g., the bipolar cells of the retina; Grossberg, 1987b; Werblin, 1971). The equations for the READ II circuit are as follows.

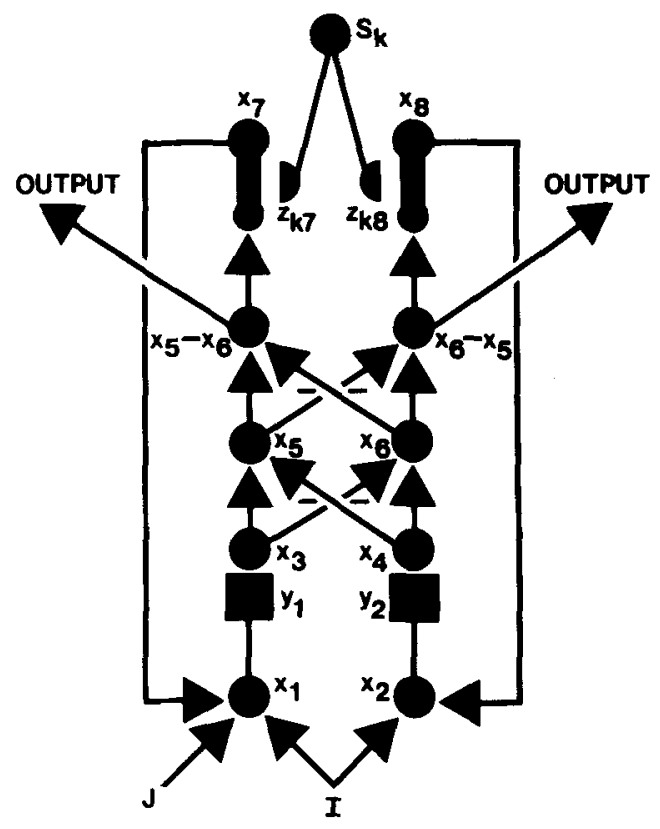

Figure 10. A READ II circuit. This circuit is mathematically equivalent to the RFAD I circuit depicted in Figure 3. In a READ II circuit, competitive normalitention and competitive opponeet procesesing are separated into two successive inhlbitory stages rather than being lumped into one stage, as in the READ I circuit. See text for details.

\section{READ II EQUATIONS}

Equations 4-9 are the same as in the READ I circuit. The next equation performs a pure normalization, without opponent processing, due to the choice $F=0$ of its inhibitory saturation point.

\section{Normalized On-Activation}

$$
\frac{d}{d t} x_{3}=-A x_{3}+\left(E-x_{3}\right) x_{3}-x_{5} x_{4}
$$

\section{Normalized Off-Activation}

$$
\frac{d}{d t} x_{6}=-A x_{6}+\left(E-x_{6}\right) x_{4}-x_{6} x_{3} .
$$

These normalized activations compete at the next processing stage to generate normalized opponent activations. Thus the variables $x_{5}-x_{6}$ and $x_{6}-x_{5}$ play the same role in the READ II circuit as do variables $x_{5}$ and $x_{6}$, respectively, in the READ I circuit. For notational simplicity, we do not represent the cells at which the opponent interactions occur as a separate stage, although this is implicit in the equations. The remaining equations of the READ II circuit are as follows.

\section{On-Activation by CS Inputs}

$$
\frac{d}{d t} x_{7}=-A x_{7}+G\left[x_{5}-x_{6}\right]^{+}+L \sum_{k} S_{k} z_{k 7}
$$


Off-Activation by CS Inputs

$$
\frac{d}{d t} x_{8}=-A x_{8}+G\left[x_{6}-x_{5}\right]^{+}+L \sum_{k} S_{k} z_{k 8}
$$

On-Conditioned Reinforcer Learning

$$
\frac{d}{d t} z_{k 7}=S_{k}\left(-K z_{k 7}+L\left[x_{5}-x_{6}\right]^{+}\right)
$$

Off-Conditioned Reinforcer Learning

$$
\frac{d}{d t} z_{k 8}=S_{k}\left(-K z_{k 8}+L\left[x_{6}-x_{5}\right]^{+}\right)
$$

\section{On-Output Signal}

$$
O_{1}=\left[x_{5}-x_{6}\right]^{+}
$$

Off-Output Signal

$$
O_{2}=\left[x_{6}-x_{5}\right]^{+}
$$

\section{Comparison With Alternative Conditioning Models}

Although the READ II circuit is mathematically equivalent to the READ I circuit, its equations make it easier to understand one of the circuit's key properties. In the associative equations 43 and 44 , conditioned reinforcer learning is driven by the terms $\left[x_{5}-x_{6}\right]^{+}$and $\left[x_{6}-x_{5}\right]^{+}$. Thus learning occurs only if the net balance of all inputs to the gated dipole favors the on-channel or the offchannel. Expressed in another way, LTM changes occur only if an increment occurs above a baseline of activation.

A number of models have been formulated to express this type of intuition. Whereas the Rescorla and Wagner (1972) and the Sutton and Barto (1981) models have attempted to represent all the factors that control the conditioning process by using a single equation for learning by individual LTM traces, the Pearce and Hall (1980) model uses several equations: one for computing the attentional parameters, one for excitatory associations, and one for inhibitory associations. Grossberg (1982a) itemized a number of basic experiments that these models cannot explain because they lump too many processes together.

The READ II equations demonstrate in a real-time setting that all of these models have attempted to express an important processing insight. The READ II equations also emphasize, however, that qualitatively different types of processes, such as gated-dipole opponent processes and CS-gated associative learning processes, interact with one another to generate these properties as an emergent property of the entire circuit, rather than as a direct property of a single synapse. This conclusion was also explicit in the READ circuit equations that were originally introduced in Grossberg (1972b) and further developed in Grossberg (1975). These circuits have stood the test of time and of subsequent data. Their further development in this article through systematic computer simulations demonstrates their robust ability to generate real- time conditioning profiles that other conditioning models have not yet been able to explain.

\section{Presynaptic Gating Versus}

Postsynaptic-to-Presynaptic Feedback

The READ III circuit depicted in Figure 11 is both physically and mathematically distinct from the READ I and READ II circuits, but its functional properties in computer simulations are remarkably similar, both qualitatively and quantitatively, to those of the READ I and II circuits. In the READ I and II circuits, associative learning is controlled by a correlation between presynaptic and postsynaptic influences, such as $S_{k}$ and $\left[x_{5}\right]^{+}$, respectively, in Equation 14. In contrast, within the READ III circuit, all of the learned changes in the LTM trace are mediated presynaptically. After describing the nature of these presynaptic influences, we also note that, in the absence of a specialized anatomical organization, their realization in vivo would be inconvenient at best.

The possibility that associative influences may be mediated presynaptically in some neural systems is consistent with some invertebrate data (Hawkins, Abrams, Carew, \& Kandel, 1983). On the other hand, both invertebrate and vertebrate associative learning data also support the existence of postsynaptic influences (Alkon, 1979, 1984a, 1984b; Levy, 1985; Levy et al., 1983; Levy \& Desmond, 1985; Rauschecker \& Singer, 1979; Singer, 1983), and some associative properties, by their very definition, require a postsynaptic influence (Grossberg \& Levine, in

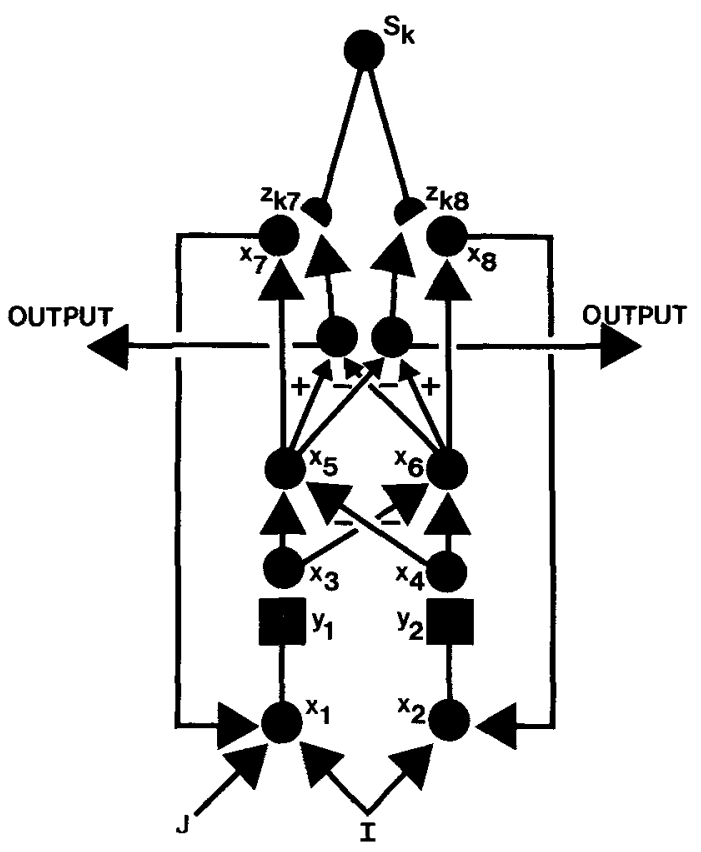

Figure 11. A READ III circuit. Unlike the READ I and II circuits, learning in a READ III circuit is driven by the correlation of two presynaptic signals, rather than by the correlation of a presynaptic signal with a postsynaptic signal. Computer simulations of both types of circuits generate similar results. 
press). Thus we present the READ III circuit to demonstrate that the simulated conditioning properties we report do not, in themselves, rule out a purely presynaptic site for conditioning.

\section{READ III EQUATIONS}

Equations 4-9, 39-40, and 43-46 are the same as in the READ II circuit. The READ II and III circuits differ only in their equations for activation by CS inputs. In both the READ I and READ II circuits, the potentials $x_{7}$ and $x_{8}$ are influenced by normalized opponent signals from the prior processing stage of the gated dipole. In the READ III circuit, potentials $x_{7}$ and $x_{8}$ are influenced by normalized, but not opponent, signals from the prior processing stage.

\section{On-Activation by CS Inputs}

$$
\frac{d}{d t} x_{7}=-A x_{7}+G\left[x_{5}\right]^{+}+L \sum_{k} S_{k} z_{k 7}
$$

\section{Off-Activation by CS Inputs}

$$
\frac{d}{d t} x_{8}=-A x_{8}+G\left[x_{6}\right]^{+}+L \sum_{k} S_{k} z_{k 8}
$$

The normalization stage, defined by Equations 39 and 40 , ensures that the potentials $x_{5}$ and $x_{6}$ compute ratios that are passed along the gated-dipole on-channel and offchannel via Equations 47 and 48 . In addition, these nonnegative output signals activate an opponent-processing stage to generate the output signals given in Equations 45 and 46. As in Figure 11, these output signals are relayed along a bifurcating pathway. One branch of the pathway carries outputs to other circuits. The other branch has a presynaptic modulatory effect on the LTM trace of its channel, as in Equations 43 and 44.

In this circuit, a single output signal, say $O_{1}$ in the onchannel, must presynaptically modulate the LTM traces $z_{k 7}$ of all the sensory representations whose signals $S_{k}$ can sample the on-channel. In order to meet this requirement, either there exists a very large number of specific pathways branching from each READ III output pathway to the synaptic terminals of all CS-activated pathways, or all these synaptic terminals are grouped together functionally so that a single modulatory signal generated by each output pathway can spread to all the synaptic terminals that abut on its channel. Other things being equal, it seems far simpler, as in the READ I and II circuits, to allow postsynaptic-to-presynaptic signals to influence each abutting synaptic terminal via a direct local feedback process.

\section{Computer Simulations With the \\ Read I Circuit: No Passive Extinction}

In each series of computer simulations, numerical parameters of the circuit were held fixed and several different experiments, characterized by different pairings of CS and US inputs, were simulated. Then individual parameters were altered and another complete series of simulations was undertaken. In this way, an understanding of how each parameter influences network dynamics was achieved. This and the next section summarize illustrative sets of computer simulations. Although the simulation sets demonstrate the formal competence of READ circuits, they are not presumed to embody the full neural machinery engaged during conditioning. The results are, we suggest, necessary but not sufficient to explain conditioning in vivo. These simulation results are used in Part II, along with other modeling results, to suggest qualitative explanations of some difficult conditioning data. In particular, in Part II (Section 24) we show how the process of opponent extinction can extinguish LTM traces actively even if parameters are set, as in this section, to prevent the LTM traces from extinguishing passively.

The simulation series reported below tested the response of a READ circuit to the five experimental combinations of CS and US inputs described in Figure 12. Figure 12a summarizes the CS and US inputs used to study primary excitatory conditioning and extinction. In these simulations, $\mathrm{CS}_{1}$ onset preceded US onset for 10 acquisition trials. Then the $\mathrm{CS}_{1}$ was presented alone for 10 extinction trials. In such a READ circuit, one mechanism of extinction is passive decay of conditioned reinforcer LTM strength when the CS is active. We show that such decay may occur in some parameter ranges, but that essentially perfect conditioned reinforcer memory obtains in other parameter ranges wherein the full range of desirable circuit properties, notably large antagonistic rebounds, prevails. Thus CS-contingent passive extinction may occur in some neural systems or species, but not others, due to evolutionary selection of a different choice of parameters. In circuits wherein passive extinction does not occur, an active extinction process may be controlled by auxiliary circuits (Grossberg, 1982c, 1987a). These auxiliary circuits match a learned expectation against the sensory events that actually occur. A mismatch may trigger a novelty reaction, which causes a burst of nonspecific arousal that can elicit an antagonistic rebound within the READ circuit. Conditioned reinforcer learning of an antagonistic rebound within an off-channel can competitively inhibit prior conditioned reinforcer learning to the corresponding on-channel due to the opponent processing that occurs between channels before the circuit elicits an output signal. This type of expectancy-mediated extinction mechanism is used to explain conditioning data in Part II.

Figure 12b summarizes the CS and US inputs used to study primary inhibitory conditioning and extinction. In these simulations, US offset preceded $\mathrm{CS}_{\mathbf{1}}$ onset for 10 acquisition trials. Then the $\mathrm{CS}_{\mathbf{1}}$ was presented alone for 10 extinction trials.

Figure $12 \mathrm{c}$ summarizes the CS and US inputs used to study secondary excitatory conditioning. In these simulations, the CS 1 preceded the US for 10 acquisition trials. Then the $\mathrm{CS}_{1}$ and the $\mathrm{CS}_{2}$ occurred together for 10 second- 


\section{$\mathrm{CS}_{1}$ \\ US}
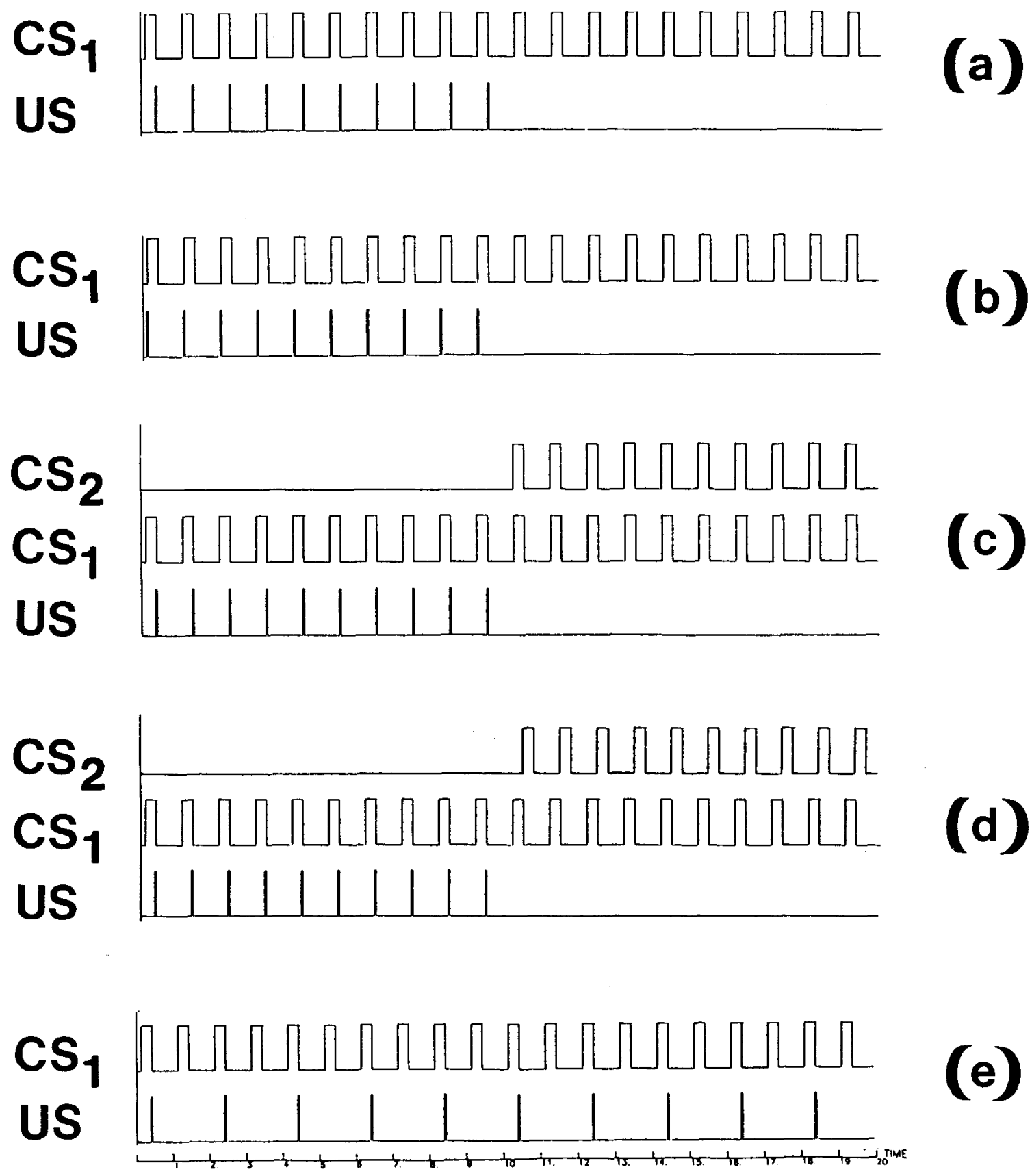

Figure 12. Input series in real time that were used in computer simulations: (a) primary excitatory conditioning and extinction; (b) primary inhibitory conditioning and extinetion; (c) secondary excitatory conditioning; (d) secondary inhibitory conditioning; (e) partial reinforcement.

ary conditioning trials, thereby conditioning the $\mathrm{CS}_{2}$. Since an isolated READ circuit does not include the limited-capacity attentional mechanisms that regulate blocking and overshadowing (Grossberg, 1982c, 1987a; Grossberg \& Levine, in press), the $\mathrm{CS}_{2}$ is not blocked by the $\mathrm{CS}_{\mathbf{1}}$ when they are simultaneously presented. Simultaneous presentation of $\mathrm{CS}_{1}$ and $\mathrm{CS}_{2}$ has much the same effect on READ circuit secondary conditioning as would onset of a sustained $\mathrm{CS}_{1}$ before onset of the $\mathrm{CS}_{2}$.
Simultaneous presentation is therefore reported here for simplicity.

Figure 12d summarizes the CS and US inputs used in secondary inhibitory conditioning. Here, the $\mathbf{C S}_{1}$ was paired with the US for 10 acquisition trials. Then $\mathrm{CS}_{1}$ offset preceded $\mathrm{CS}_{2}$ onset for 10 secondary conditioning trials.

Figure 12e describes the CS and US inputs used in excitatory partial reinforcement. In these simulations, $\mathrm{CS}_{1}$ 
and US pairing alternated with presentation of the $\mathrm{CS}_{1}$ alone for 20 trials. As noted above, the conditioning that occurs within an isolated READ circuit, whether due to continuous reinforcement or partial reinforcement, is not modulated by expectation mechanisms. Such modulation can yield higher asymptotic response levels under partial reward than under continuous reward (Grossberg, 1975; reprinted in Grossberg, 1982c), as also occurs in many experimental paradigms (Boren, 1961; Brogden, 1939; Felton \& Lyon, 1966; Gibbon, Farrell, Locurto, Duncan, \& Terrace, 1980; Gibbs, Latham, \& Gormezano, 1978; Gonzalez, 1973, 1974; Perkins et al., 1975; Schwartz \& Williams, 1972; Wasserman, 1974; Wasserman, Hunter, Gutowski, \& Bader, 1975). This type of enhancement effect does not occur in an isolated READ circuit. The discussion in Part II describes how the interaction of expectation mechanisms with READ circuit mechanisms can yield higher asymptotes and more resistant extinction during partial reward than during continuous reward.

Figures 13-17 depict a series of simulations using a fixed set of numerical parameters. Each curve depicts the real-time behavior of an activation (STM trace) or adaptive weight (LTM trace) of the READ I circuit. Due to the fact that each variable fluctuates over a different range of numerical values, each curve has been normalized to fit within an interval of fixed height. We call particular attention to the following features of these conditioning curves.

Consider Figure 13 for definiteness. This figure depicts a simulation of excitatory primary conditioning using the inputs in Figure 12a. Because the US is presented to the on-channel, the on-transmitter $y_{1}$ in Figure 13 undergoes a series of habituation-accumulation cycles on successive learning trials, as schematized in Figure 6. Due to these reactions, the on-activations throughout the circuit undergo overshoot-habituation-undershoot-habituation cycles through time, also schematized in Figure 6 . The variables $x_{5}$ and $x_{6}$ in Figure 13 illustrate these properties.

The variable $\mathrm{CS}_{1}-\mathrm{ON}$ describes conditioning of the LTM trace within the pathway from the sensory representation of the $\mathrm{CS}_{1}$ to the on-channel of the READ circuit. Notice that after the 10 acquisition trials terminate, future presentations of the $\mathrm{CS}_{1}$ alone on extinction trials do not cause delay of the $C S_{1}-O N$ LTM trace. For this choice of parameters, memory is essentially perfect. Forgetting is due to active relearning, notably counter-conditioning of $\mathrm{CS}_{1}$-OFF, as in the interference theory of forgetting (Adams, 1967; Grossberg, 1972b).

Another important feature of circuit dynamics is seen in the output functions $\left[x_{5}\right]^{+}$and $\left[x_{6}\right]^{+}$of the on-channel and off-channel, respectively. Because the output signals are rectified, they generate sustained but habituative onreactions and transient off-reactions, as schematized in Figure 5.

Figure 14 summarizes a simulation of inhibitory primary conditioning obtained through a backward conditioning procedure, using the inputs depicted in Fig- ure $12 \mathrm{~b}$. In this simulation, the LTM trace $\mathrm{CS}_{1}-\mathrm{OFF}$ is the one that learns. This is the LTM trace in the pathway from the sensory representation of the $\mathrm{CS}_{1}$ to the offchannel of the READ circuit. Conditioning of the offchannel is due to the antagonistic rebounds that occur after the US to the on-channel is terminated. These antagonistic rebounds, in turn, are caused by the habituation of the transmitter gate $y_{1}$ in the on-channel. Note that these rebounds also cause habituation of the transmitter gate $y_{2}$ in the off-channel, but that $y_{2}$ habituates during time intervals when $y_{1}$ is recovering.

An important point of comparison between Figures 13 and 14 concerns the maximum sizes achieved by the conditioned LTM traces $\mathrm{CS}_{1}-\mathrm{ON}$ and $\mathrm{CS}_{1}-\mathrm{OFF}$, respectively. These maximum sizes ( 35.8 and 55 , respectively) are commensurate. The existence of relatively large values of off-LTM traces tended to covary in our simulations with the persistence of memory during extinction trials. Large off-LTM traces and good memory went hand in hand. It is still too soon to say whether this is a general property of READ circuits, and thus a property upon which an experimental prediction can securely be based. On the other hand, it is an interesting correlation that deserves further study.

In order to study the covariation of extinction with large off-rebounds, we did parametric studies, varying the feedback coefficient $M$ in Equations 31 and 32 from .01 to .07 . In Figures $13-17$, we chose $M=.05$. For this parameter choice, the off-LTM trace slowly decays to approximately $70 \%$ of its maximal value during successive presentation of the CS alone. A similar decay occurs given choices of $M$ between .01 and .05 .

Figure 15 depicts a simulation of excitatory secondary conditioning using the inputs summarized in Figure 12c. The LTM trace $\mathrm{CS}_{1}-\mathrm{ON}$ grows during the first 10 trials and is then used to induce growth of the LTM trace $\mathrm{CS}_{2}$ ON during the next 10 trials, without undermining its own LTM strength. The size (37.7) of $\mathrm{CS}_{1}-\mathrm{ON}$ after $10 \mathrm{ac}-$ quisition trials is larger than the size (33.3) of $\mathrm{CS}_{2}-\mathrm{ON}$ after 10 acquisition trials. Thus, secondary conditioning generates significant LTM strength in this READ circuit, but not LTM strength as great as that generated due to primary conditioning.

Figure 16 depicts a simulation of inhibitory secondary conditioning using the inputs summarized in Figure 12d. These simulations fully exploit the fact that the READ circuits contain positive feedback loops. Grossberg (1972b, 1975) was the first to note that in order for a CS to be conditionable either directly to the on-channel or to an antagonistic rebound in the off-channel, its LTM traces must contact the gated dipole at a stage subsequent to the habituative transmitter gates. In order for offset of a $\mathrm{CS}_{1}$ to cause an antagonistic rebound, its LTM traces must contact the gated dipole at a stage prior to the habituative transmitter gates. In order for the same stage of LTM contact with the gated dipole to occur both subsequent to and prior to the habituative transmitter gates, the gated dipole must contain positive feedback pathways. In Fig- 


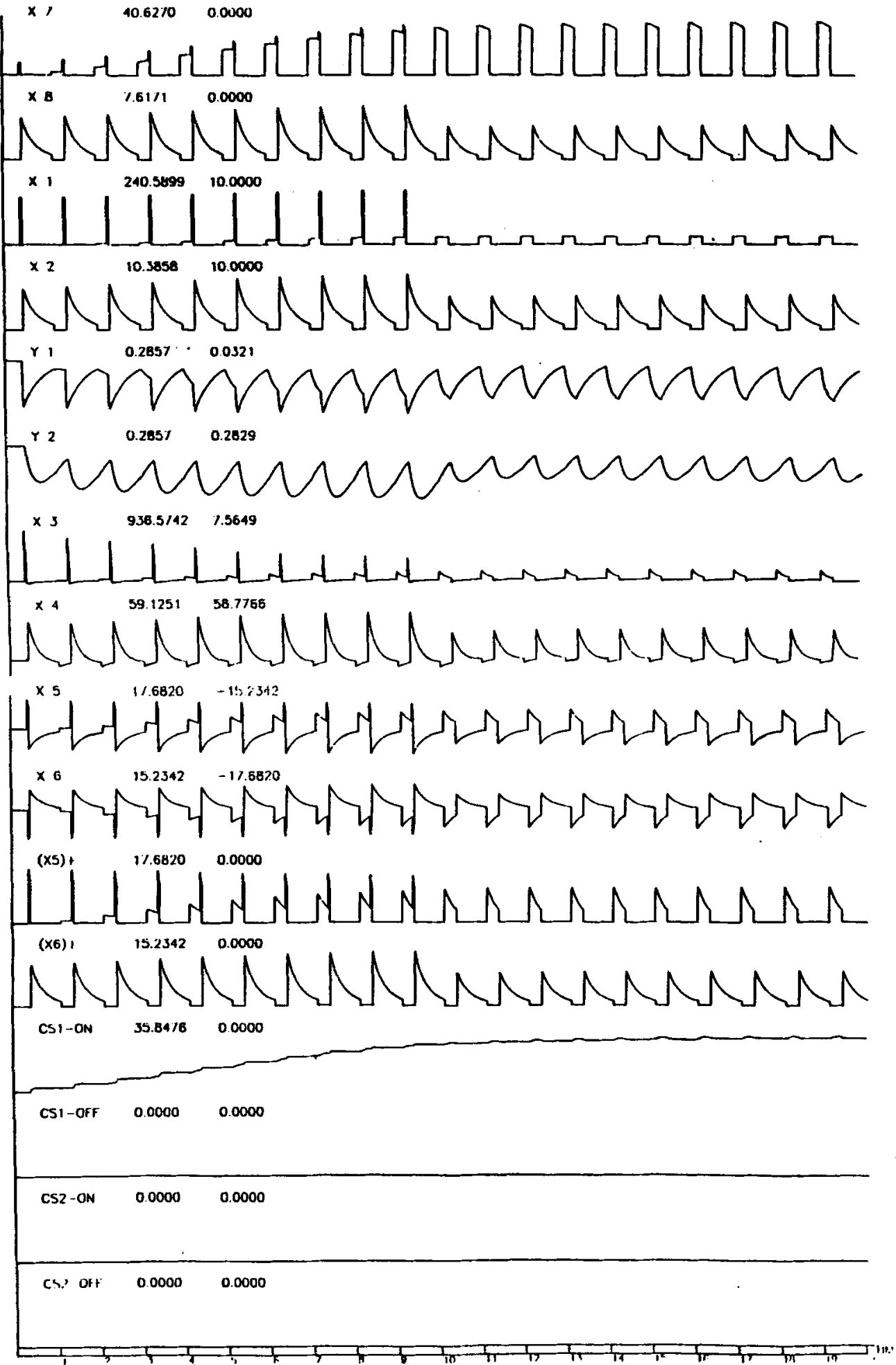

Figure 13. Computer simulation of primary excitatory conditioning and extinction with slow habituation and large feedback in a READ I circuit. The conditioned stimulus $\left(C S_{1}\right)$ is paired with the unconditioned stimulus (US) during the first 10 simulated trials, and $\mathrm{CS}_{1}$ is presented in the absence of the US in the next 10 simulated trials. The numbers above each plot are the maximum and minimum values of the plot. Parameters are $A=1, B=.005, C=.00125, D=20, E=20, F=20, G=.5, H=.005, K=.025, L=20, M=.05$. 


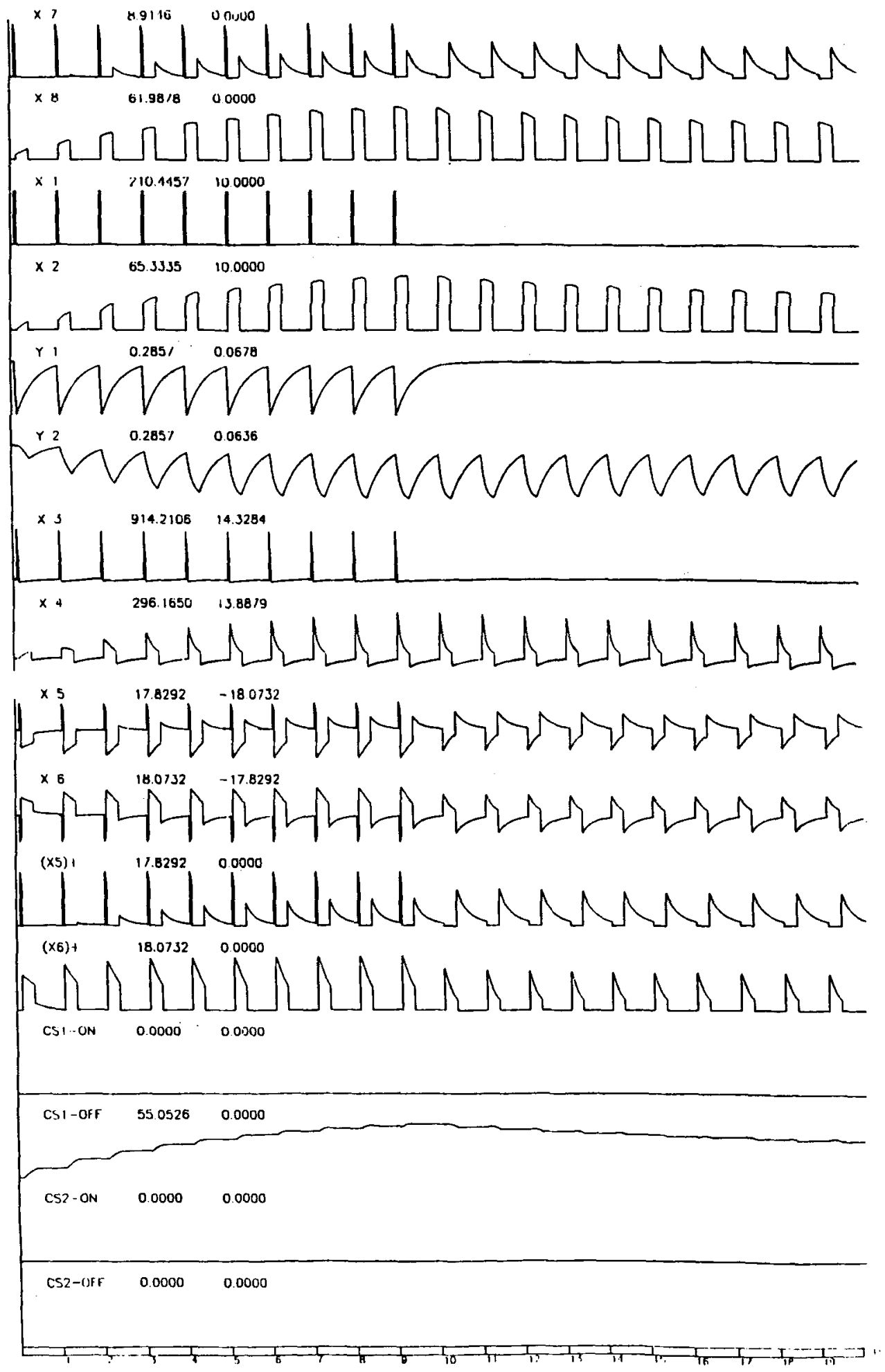

Figure 14. Computer simulation of primary inhibitory conditioning and extinction with slow habituation and large feedback in a READ I circuit. The conditioned stimulus $\left(\mathrm{CS}_{1}\right)$ is presented after the offset of the unconditioned stimulus (US) during the first 10 simulated trials, and $\mathrm{CS}_{1}$ is presented in the absence of the US in the next 10 simulated trials. The parameters are those given in Figure 13. 


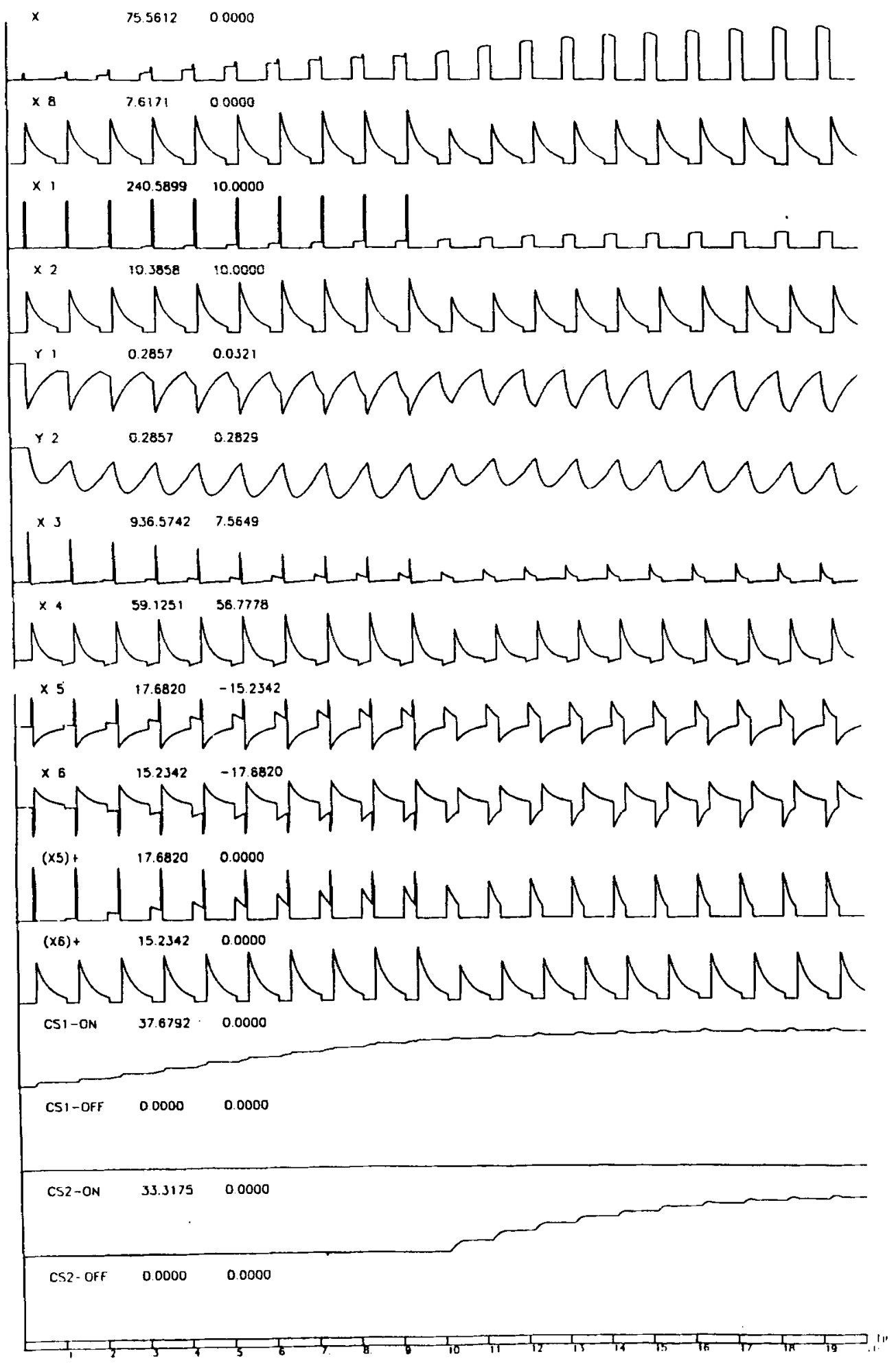

Figure 15. Computer simulation of secondary excitatory conditioning with slow habituation and large feedback in a READ I circuit. The first conditioned stimulus $\left(\mathrm{CS}_{1}\right)$ is presented with the unconditioned stimulus (US) during the first 10 simulated trials, and $\mathrm{CS}_{1}$ is presented with $C S_{2}$ in the absence of the US in the next 10 simulated trials. The parameters are those given in Figure 13. 

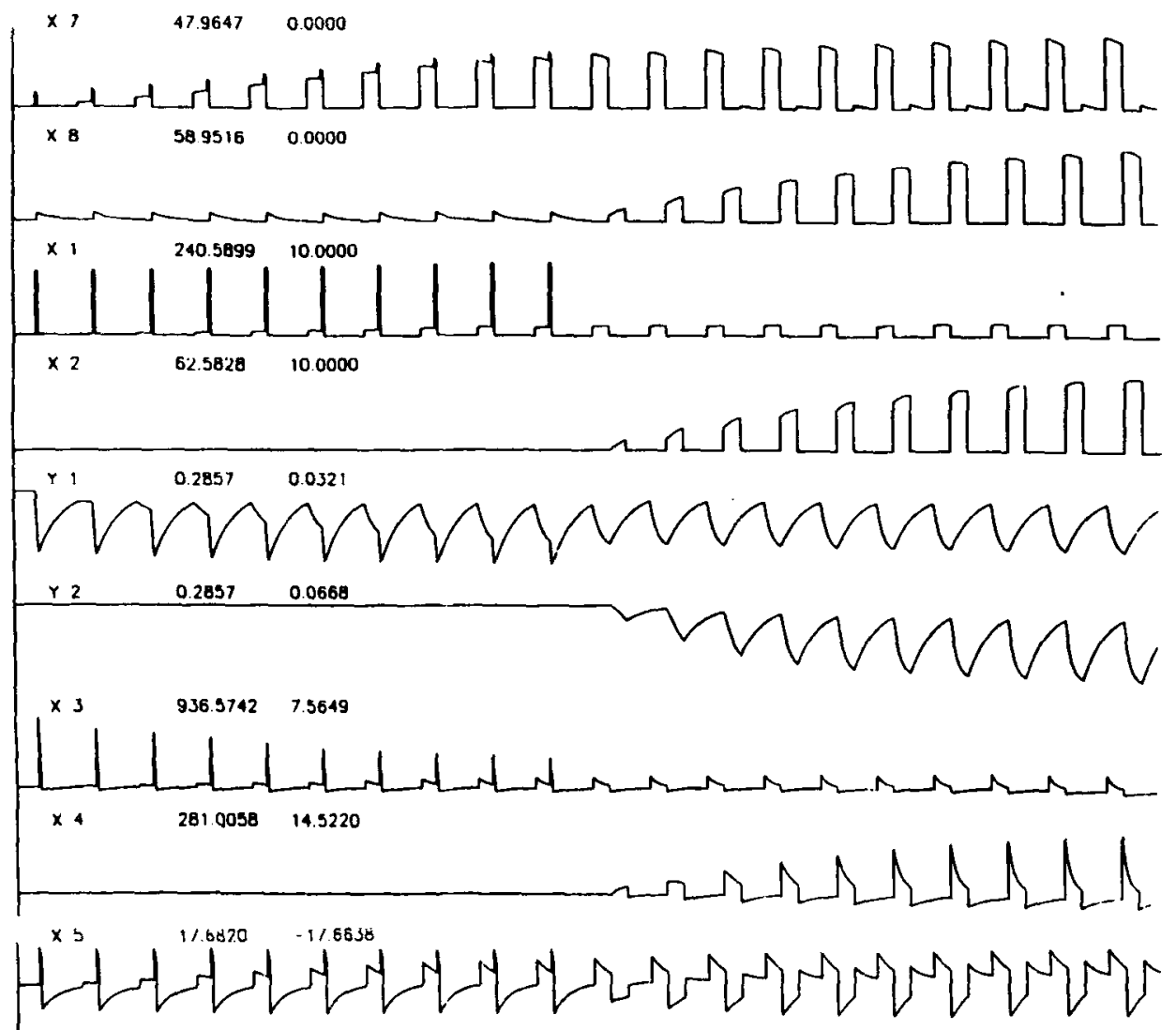

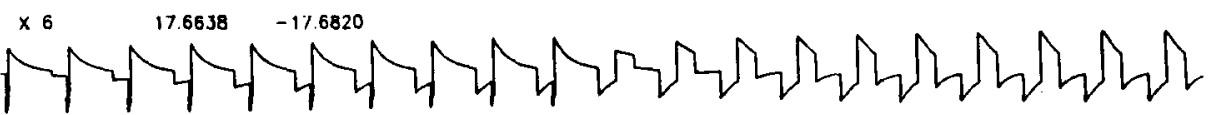

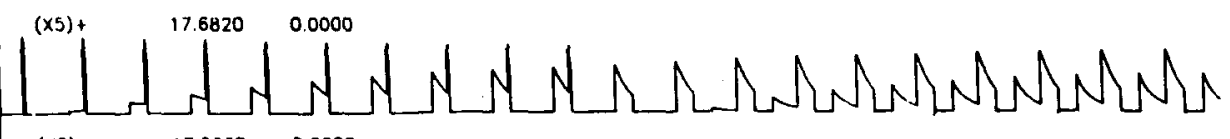

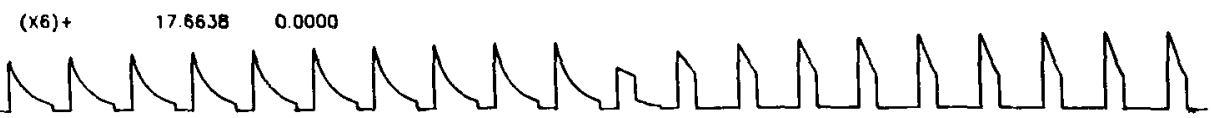
CSI-ON $\quad \mathbf{4 2 . 3 8 9 6 \quad 0 0 0 0 0}$ CS1-OFF $0.0000 \quad 0.0000$

\begin{tabular}{|c|c|c|}
\hline $\mathrm{CS} 2 \mathrm{CN}$ & 0.0000 & 00000 \\
\hline CS? Of $\mathrm{F}$ & 52.3160 & 0.0000 \\
\hline
\end{tabular}

Figure 16. Computer simulation of secondary inhibitory conditioning with slow habituation and large feedback in a READ I circuit. The first conditioned stimulus $\left(\mathrm{CS}_{1}\right)$ is presented with the unconditioned stimulus (US) during the first 10 simulated trials, and $\mathrm{CS}_{2}$ is presented after $\mathrm{CS}_{1}$ ofiset in the absence of the US in the next 10 simulated trials. The parameters are those given in Figure 13. 
ure $16, \mathrm{CS}_{1}-\mathrm{ON}$ grows on the first 10 trials, due to pairing with the US, whereas $\mathrm{CS}_{2}$-OFF grows on the next 10 trials, due to pairing with the antagonistic rebound caused by the offset of $\mathrm{CS}_{1}$. The maximum size (42.4) of $\mathrm{CS}_{1}$ ON during the first 10 trials is smaller than the maximum size (52.3) of $\mathrm{CS}_{2}$-OFF during the next 10 trials.

Figure 17 describes a simulation using the partialreward schedule described in Figure 12e. The rate of acquisition is less than that in the continuous-reward case of Figure 13. Because memory is essentially perfect during passive extinction trials, the asymptotic associative strength can grow to close to that achieved using continuous reward. Because this READ circuit is not linked to expectation mechanisms, the nonoccurrence of an expected US, or the occurrence of an unexpected US, on later conditioning trials has no influence on the course of conditioning when the CS is presented alone.

Key properties of these computer simulations tend to be supported by experimental data. These simulations do not incorporate a number of the model's attentional and expectancy mechanisms used to analyze data in Part II, but their properties are consistent with data wherein such mechanisms do not play a rate-limiting role.

In the case of excitatory conditioning (Figure 13), simulations show conditioned responses (CRs) of increasing amplitude over trials, as has often been described in classical conditioning (see, e.g., Gormezano, Kehoe, \& Marshall, 1983).

Rescorla and LoLordo (1965) and Siegel and Domjan (1971) found that backward conditioning procedures, as described in Figure 14, yield inhibitory conditioning. Zimmer-Hart and Rescorla (1974) found that inhibitory conditioning does not extinguish after presentations of the CS alone. In agreement with Zimmer-Hart and Rescorla (1974), there exists a parameter range for the READ circuit such that complete extinction of the CS-OFF association does not occur due to presentation of the CS alone.

In the READ circuit, extinction of the $\mathrm{CS}_{1}-\mathrm{ON}$ association does not affect the $\mathrm{CS}_{2}-\mathrm{ON}$ association. This result agrees with data obtained by Rizley and Rescorla (1972), who used rats as subjects in an aversive conditioning paradigm, and by Holland and Rescorla (1975), who also used rats as subjects, but in an appetitive paradigm. On the other hand, there exist several experimental paradigms (Leyland, 1977; Lysle \& Fowler, 1985; Miller \& Schachtman, 1985; Rashotte, Griffin, \& Sisk, 1977) in which extinction of a given stimulus can significantly influence the behavioral efficacy of other conditioned stimuli. In Part II, we append READ circuit mechanisms to cognitive modulatory circuits to illustrate how such an augmented circuit can be used to analyze such data.

\section{Computer Simulations in Other Parameter Ranges: Responses to Stimulus Transients and Passive Extinction}

The simulations depicted in Figures 18-21 show how speeding up the habituation and accumulation rates of the transmitter gates influences circuit dynamics. In Figures
18 and 19 , the rates chosen are twice as fast as those in Figures 13-17. In Figures 20 and 21, the rates chosen are four times as fast as those in Figures 13-17. In Figures 18 and 20 , the simulations are of excitatory secondary conditioning. In Figures 19 and 21, the simulations are of inhibitory secondary conditioning. These simulations illustrate the robustness of READ circuit properties within a physically plausible parameter range. The faster habituation rate causes a more rapidly falling overshoot in circuit activations, and thus an accentuation of transient, rather than sustained, responses to the CS and US. Otherwise, the qualitative properties of conditioning are preserved across these parameter changes.

Figures 22-26 depict a complete set of simulations in a parameter range wherein passive extinction occurs when the CS is not followed by a US. The READ circuit in which passive extinction occurs has the same parameters as the READ circuit depicted in Figures 20 and 21, with one exception: In Figures 22-26, the parameter that controls the strength of the positive feedback signals from $x_{7} \rightarrow x_{1}$ and $x_{8} \rightarrow x_{2}$ was chosen to be smaller.

Several functional properties of the READ circuit changed as a result of this single change in parameters. As already mentioned, passive extinction occurs in all of Figures 22-26. In addition, antagonistic rebounds are smaller, so inhibitory conditioning is weaker (Figures 21 and 25) relative to the corresponding level of excitatory conditioning (Figures 22 and 24). Finally, due to the passive extinction that can occur on nonrewarded trials, both the rate and the asymptote of learning are less in the partial-reward case (Figure 26) than in the continuousreward case (Figure 22), unlike the partial-reward case in which no passive extinction occurs (Figure 17). Despite these quantitative changes, the READ circuit continues to exhibit the main qualitative conditioning properties that are exhibited in its other displayed parameter ranges. These results show that the circuit's emergent properties are robust over at least four- to fivefold changes in the size of key parameters.

\section{PART II}

\section{The Relationship Between Conditioned Inhibition and Blocking Paradigms}

When a conditioned stimulus, $\mathrm{CS}_{1}$, is appropriately paired with a shock US in a conditioned-suppression paradigm, it can become a conditioned excitor, as measured by a decreased suppression ratio, an increased response latency, or other indices of conditioned fear (Lysle \& Fowler, 1985; Miller \& Schachtman, 1985). If simultaneous pairing of the $\mathrm{CS}_{1}$ with another conditioned stimulus, $\mathrm{CS}_{2}$, is followed by a no-shock interval, the $\mathrm{CS}_{2}$ can become a conditioned inhibitor, as tested by an increased suppression ratio, a decreased response latency, and so on. Conditioned inhibitors elicit a number of paradoxical behavioral properties, which have attracted intense experimental interest. 

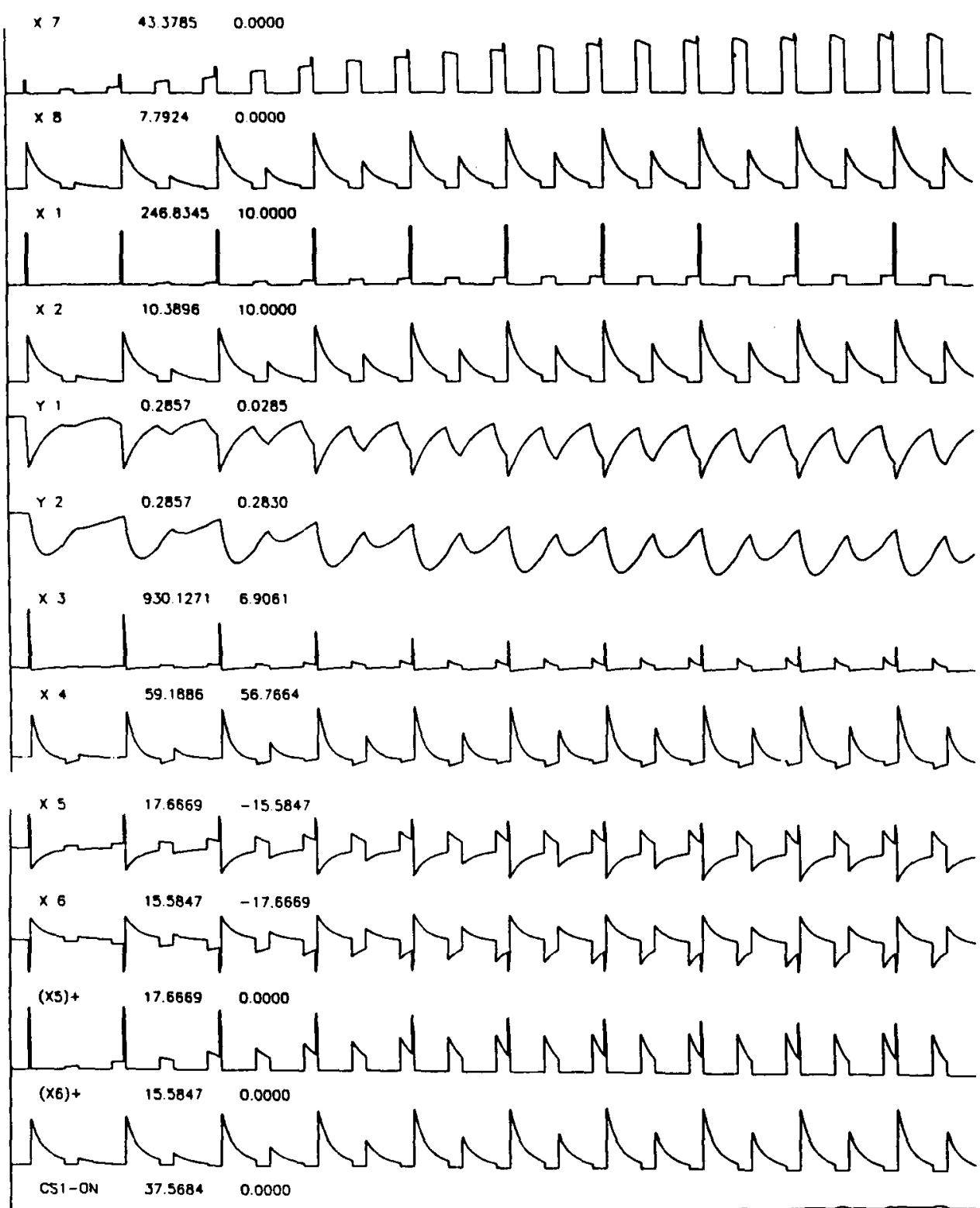

C51-OFF $.0 .0000 \quad 0.0000$

CS2-ON $\quad 0.0000 \quad 0.0000$

CS2-OFF $\quad 0.0000 \quad 0.0000$

Figure 17. Computer simulation of partial reinforcement with slow habituation and large feedback in a READ I circuit. The conditioned stimulus $\left(\mathrm{CS}_{1}\right)$ is alternately presented with the unconditioned stimulus (US) and without the US during 20 simulated trials. The parameters are those given in Figure 13. 


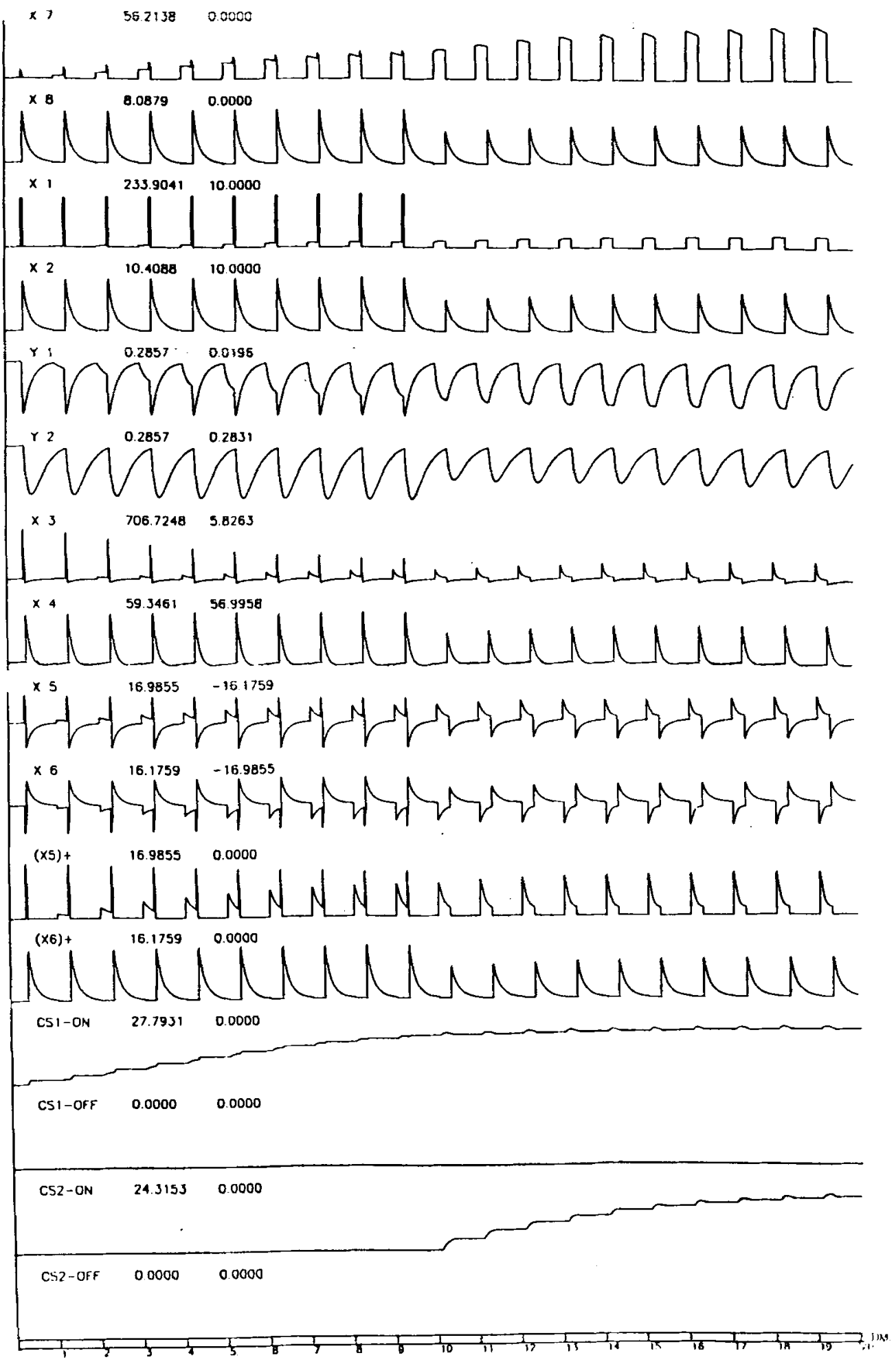

Figure 18. Computer simulation of secondary excitatory conditioning with intermediate habituation and large feedback in a READ I circuit. The first conditioned stimulus $\left(\mathrm{CS}_{1}\right)$ is presented with the unconditioned stimulus (US) during the first 10 simulated trials, and $\mathrm{CS}_{2}$ is presented with $\mathrm{CS}_{1}$ in the absence of the US in the next 10 simulated trials. The parameters are those given in Figure 13, except that $B=.010$ and $C=.0025$. 


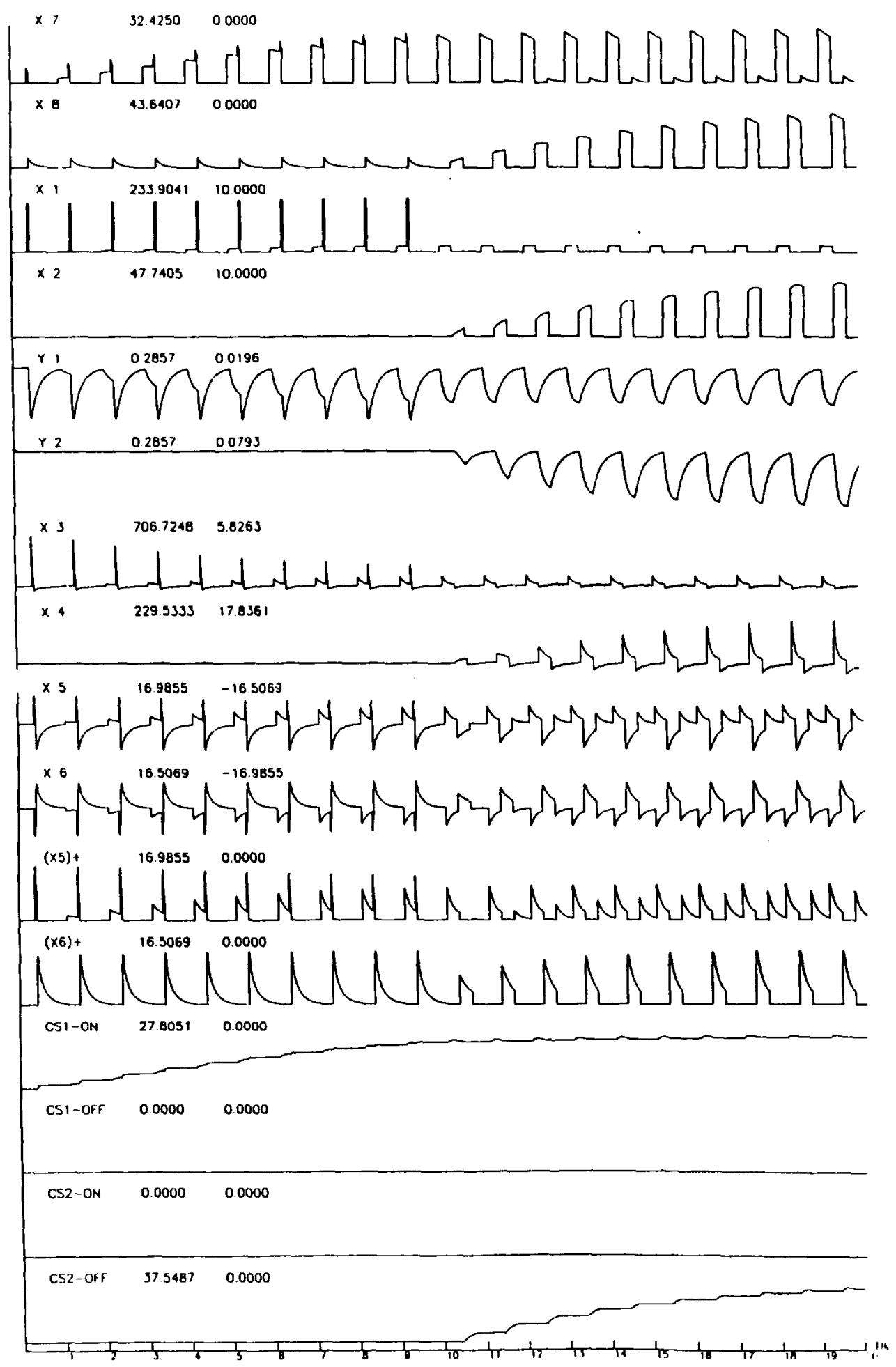

Figure 19. Computer simulation of secondary inhibitory conditioning with intermediate habituation and large feedback in a READ I circuit. The first conditioned stimulus $\left(\mathrm{CS}_{1}\right)$ is presented with the unconditioned stimulus (US) during the first 10 simulated trials, and $\mathrm{CS}_{2}$ is presented after $\mathrm{CS}_{1}$ offset in the absence of the US in the next 10 simulated trials. The parameters are those given in Figure 18. 


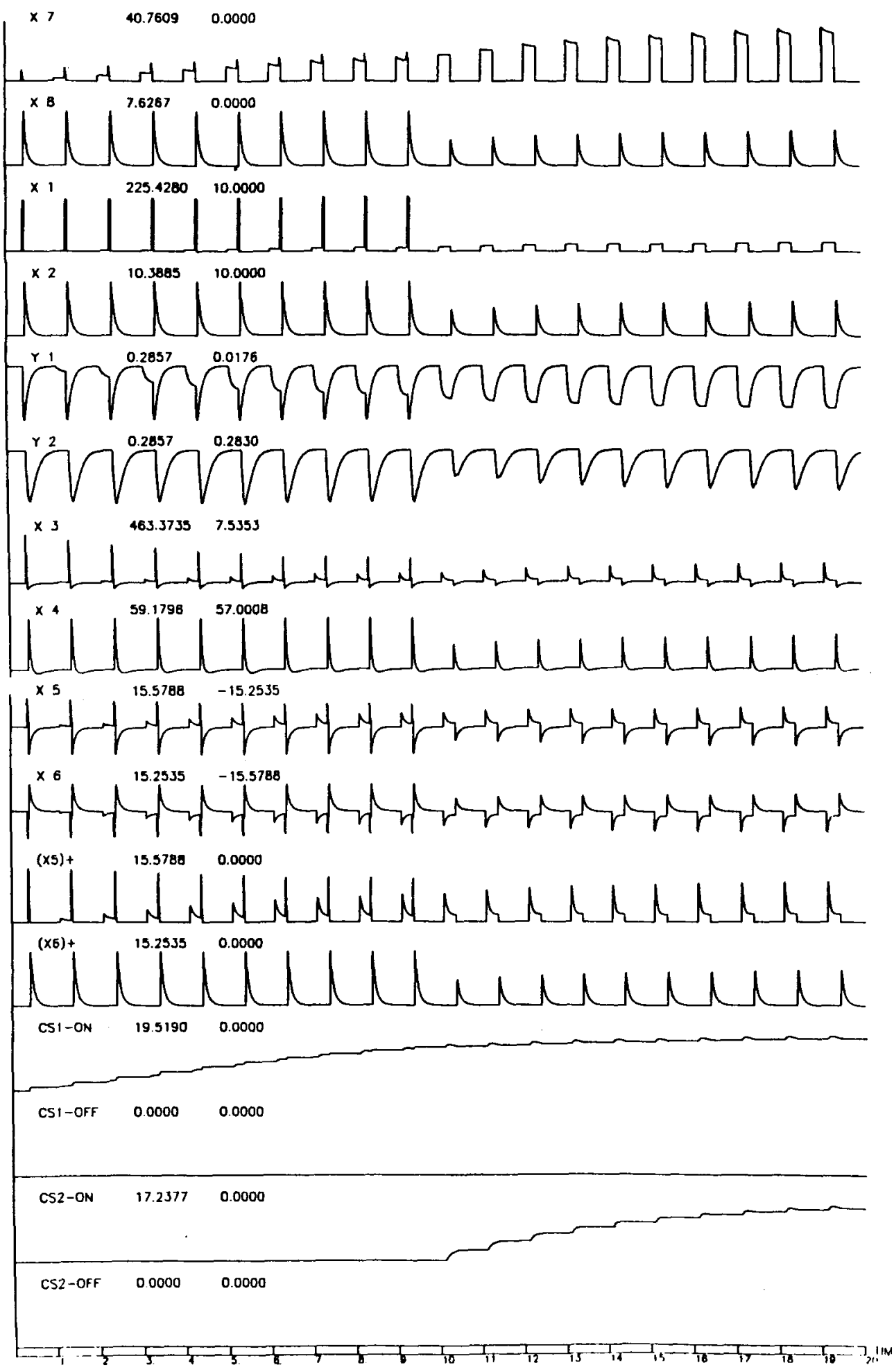

Figure 20. Computer simulation of secondary excitatory conditioning with fast habituation and large feedback in a READ I circuit. The first conditioned stimulus $\left(\mathrm{CS}_{1}\right)$ is presented with the unconditioned stimulus (US) during the first 10 simulated trials, and $\mathrm{CS}_{1}$ is presented with $\mathrm{CS}_{2}$ in the absence of the US in the next 10 simulated trials. The parameters are those given in Figure 13, except that $B=.020$ and $C=.005$. 


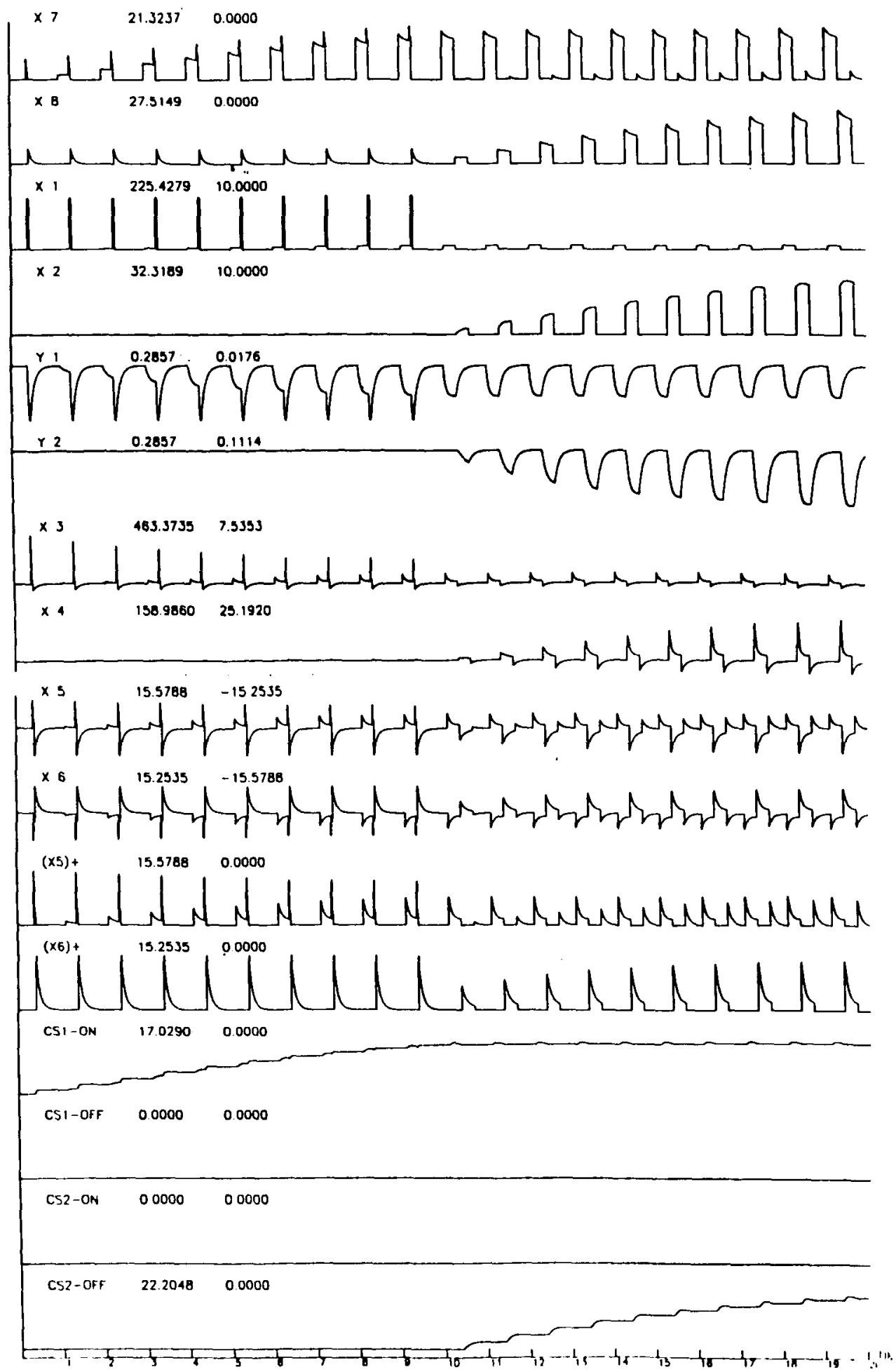

Figure 21. Computer simulation of secondary inhibitory conditioning with fast habituation and large feedback in a READ I circuit. The first conditioned stimulus $\left(\mathrm{CS}_{1}\right)$ is presented with the unconditioned stimulus (US) during the first 10 simulated trials, and $\mathrm{CS}_{2}$ is presented after $\mathrm{CS}_{1}$ offset in the absence of the US in the next 10 simulated trials. The parameters are those given in Figure 20. 


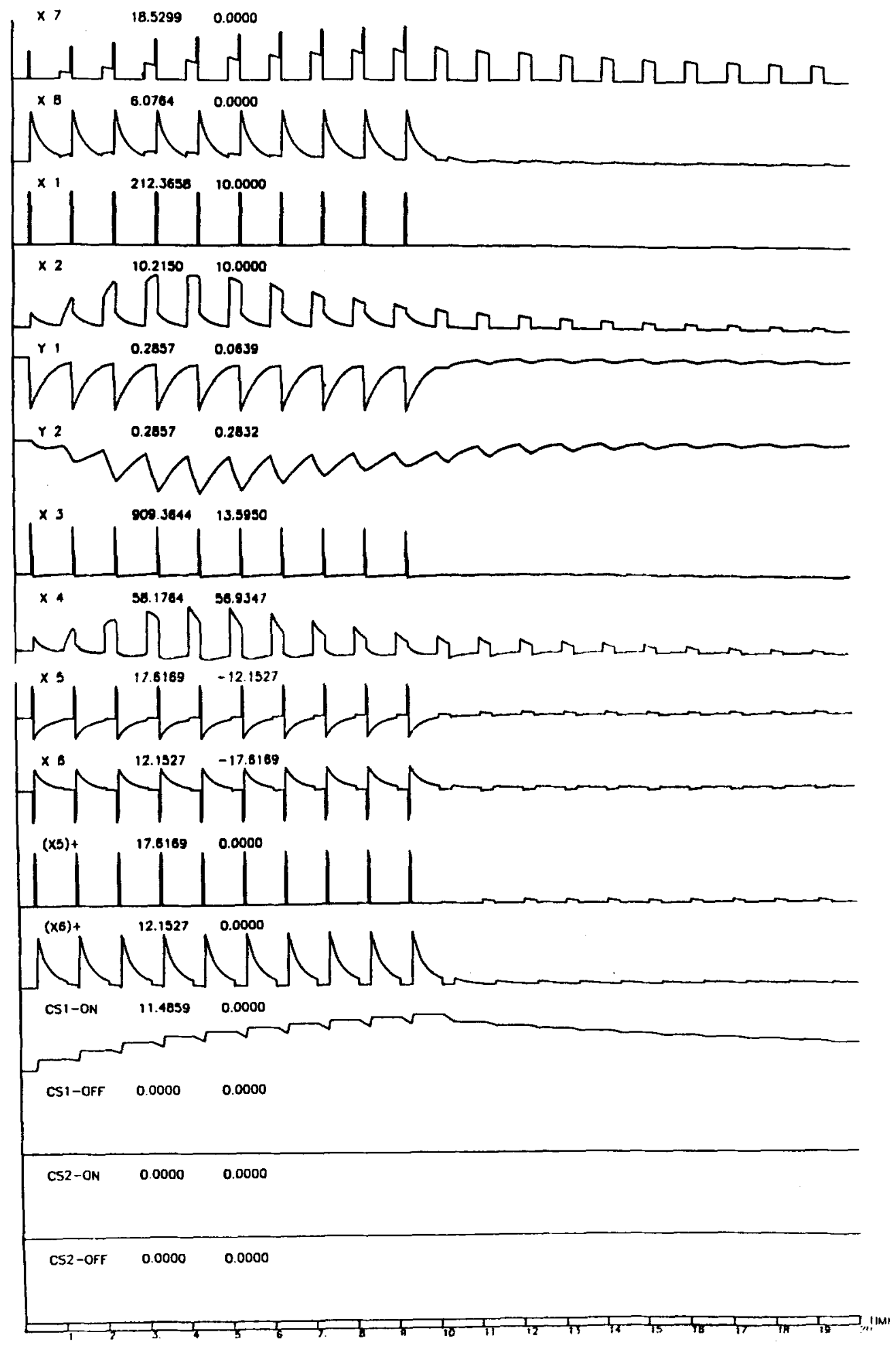

Figure 22. Computer simulation of primary excitatory conditioning and extinction with slow habituation and small feedback in a READ I circuit. The conditioned stimulus $\left(\mathrm{CS}_{1}\right)$ is paired with the unconditioned stimulus (US) during the first 10 simulated trials, and $\mathrm{CS}_{1}$ is presented in the absence of the US in the next 10 simulated trials. The parameters are those given in Figure 20, except that $M=.01$. 


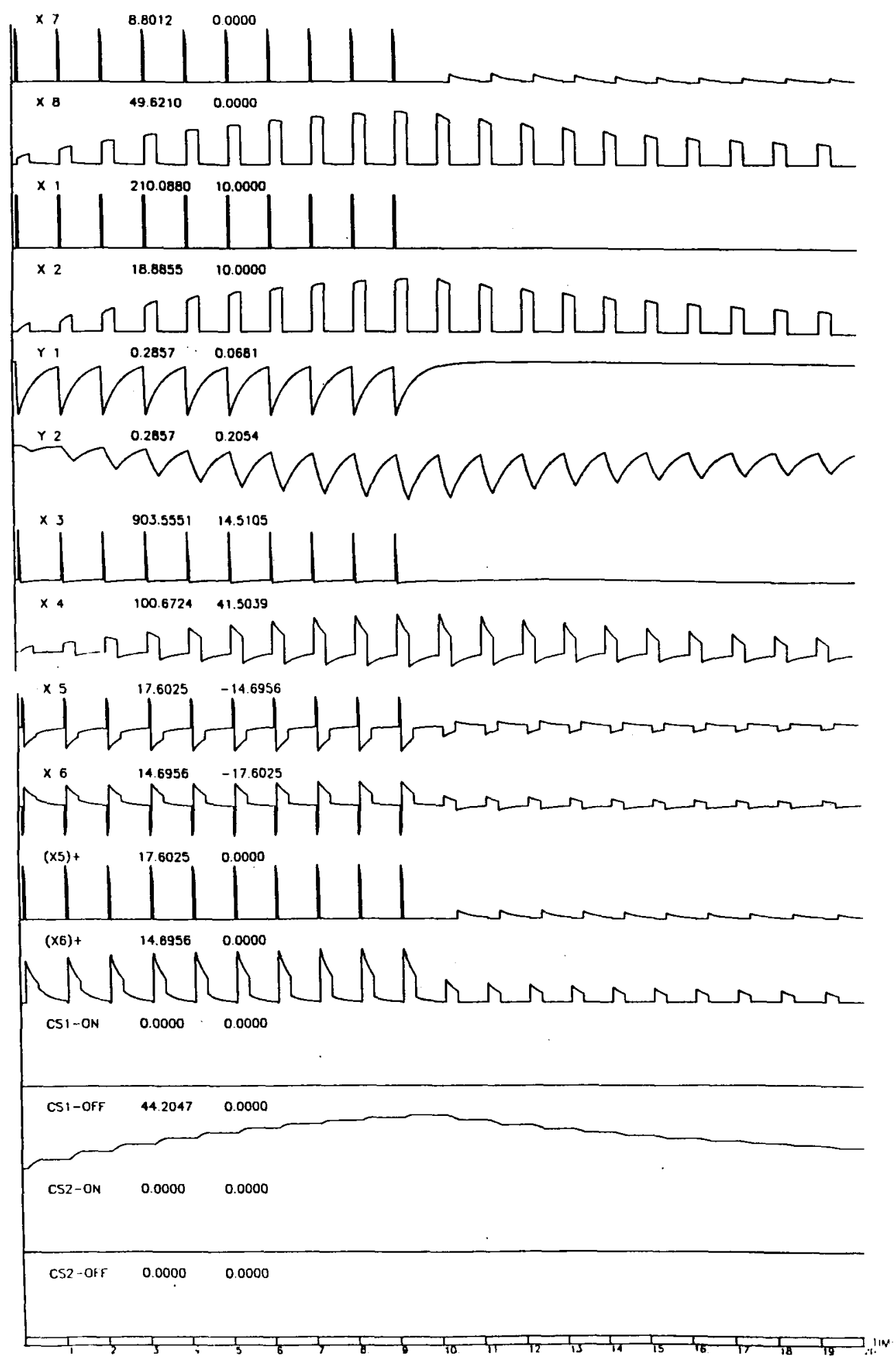

Figure 23. Computer simulation of primary inhibitory conditioning and extinction with slow habituation and small feedback in a READ I circuit. The conditioned stimulus $\left(\mathrm{CS}_{1}\right)$ is presented after the offset of the unconditioned stimulus (US) during the first 10 simulated trials, and $\mathrm{CS}_{1}$ is presented in the absence of the US in the next 10 simulated trials. The parameters are those given in Figure 22. 


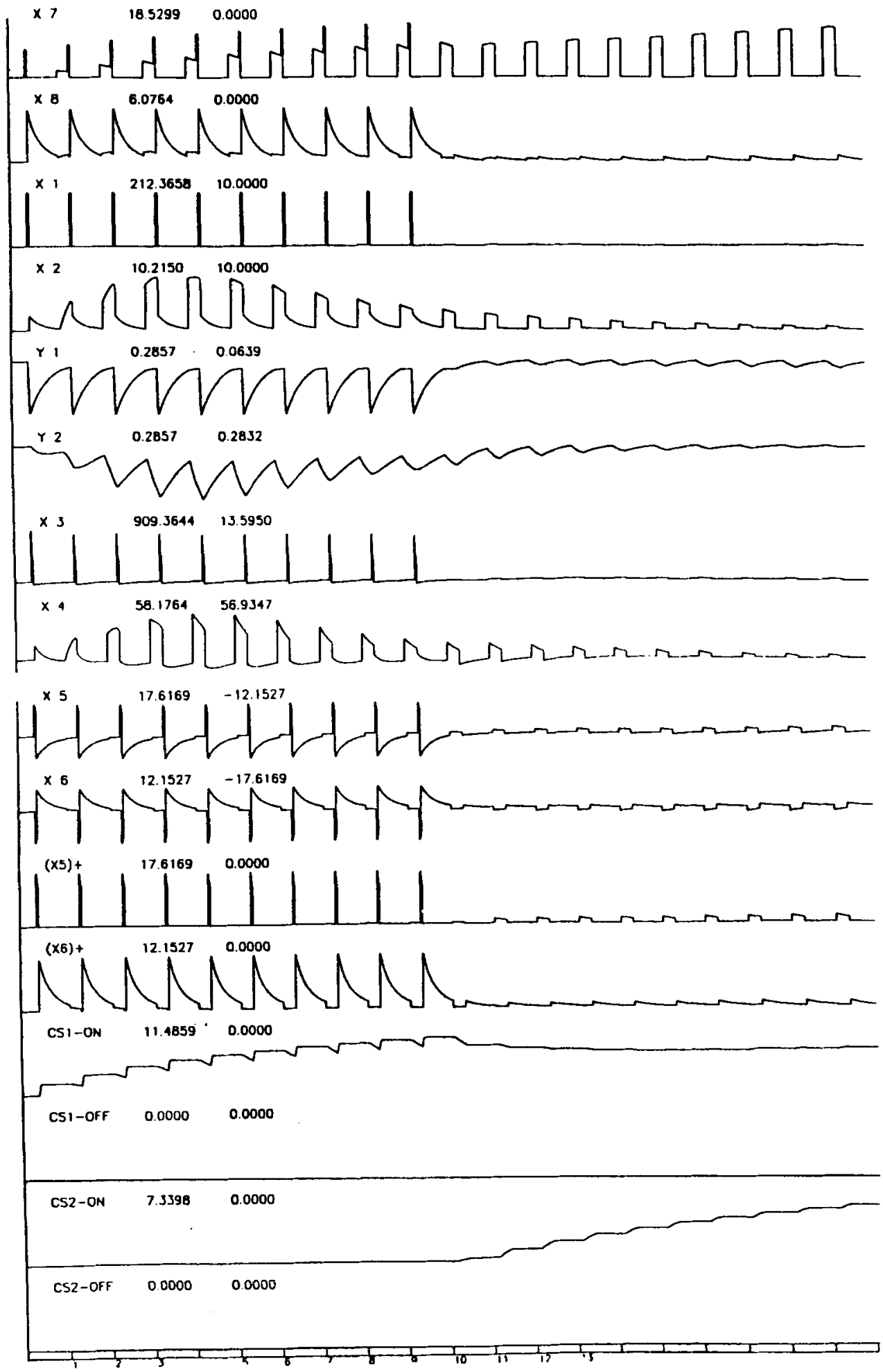

Figure 24. Computer simulation of secondary excitatory conditioning with slow habituation and small feedback in a READ I circuit. The first conditioned stimulus $\left(\mathrm{CS}_{1}\right)$ is presented with the unconditioned stimulus (US) during the first 10 simulated trials, and $\mathrm{CS}_{1}$ is presented with $\mathrm{CS}_{2}$ in the absence of the US in the next 10 simulated trials. The parameters are those given in Figure 22. 

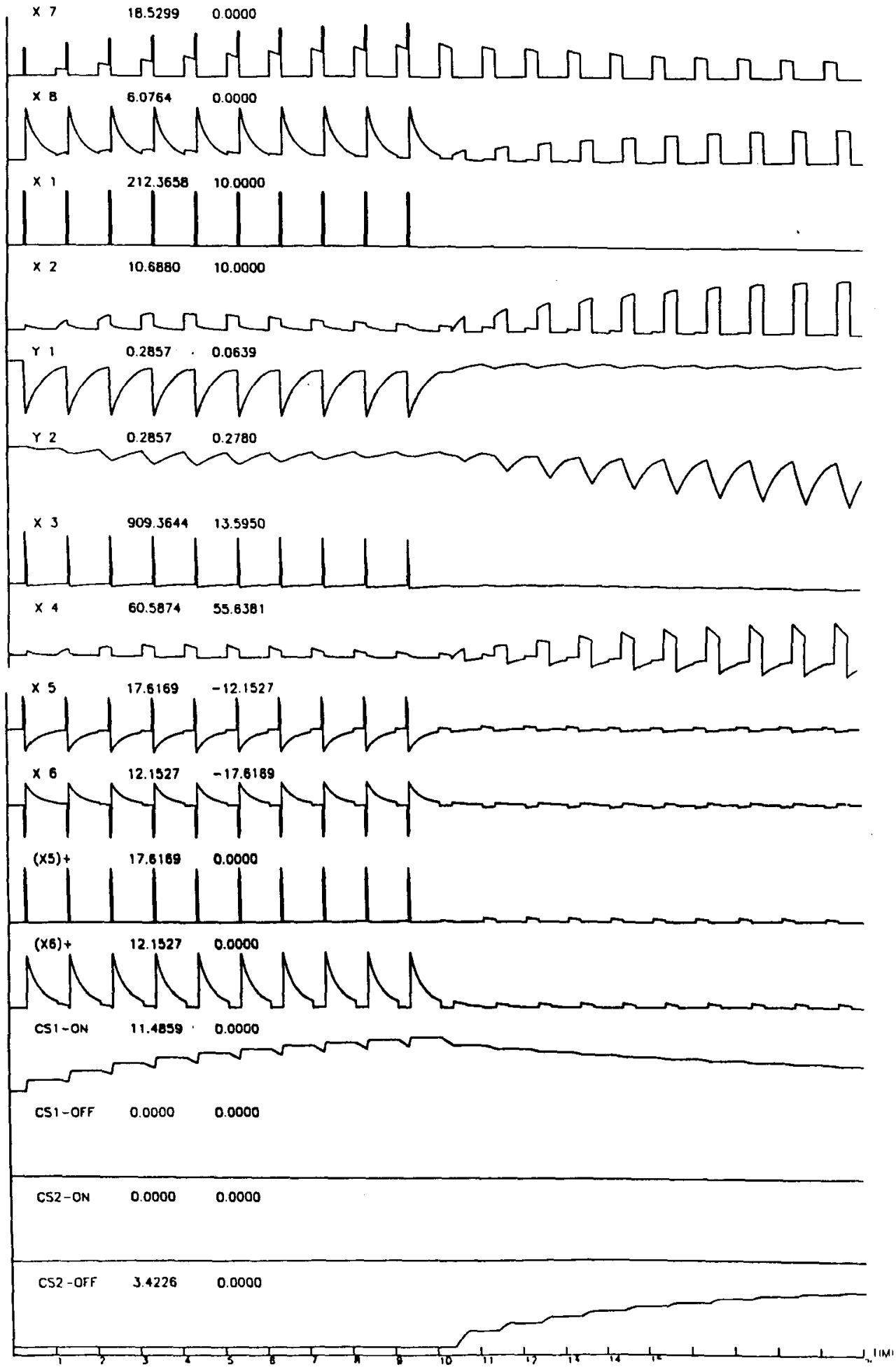

Figure 25. Computer simulation of secondary inhibitory conditioning with slow habituation and small feedback in a READ I circuit. The first conditioned stimulus $\left(\mathrm{CS}_{1}\right)$ is presented with the unconditioned stimulus (US) during the first 10 simulated trials, and $\mathrm{CS}_{2}$ is presented after $\mathrm{CS}_{1}$ offset in the absence of the US in the next 10 simulated trials. The parameters are those given in Figure 22. 

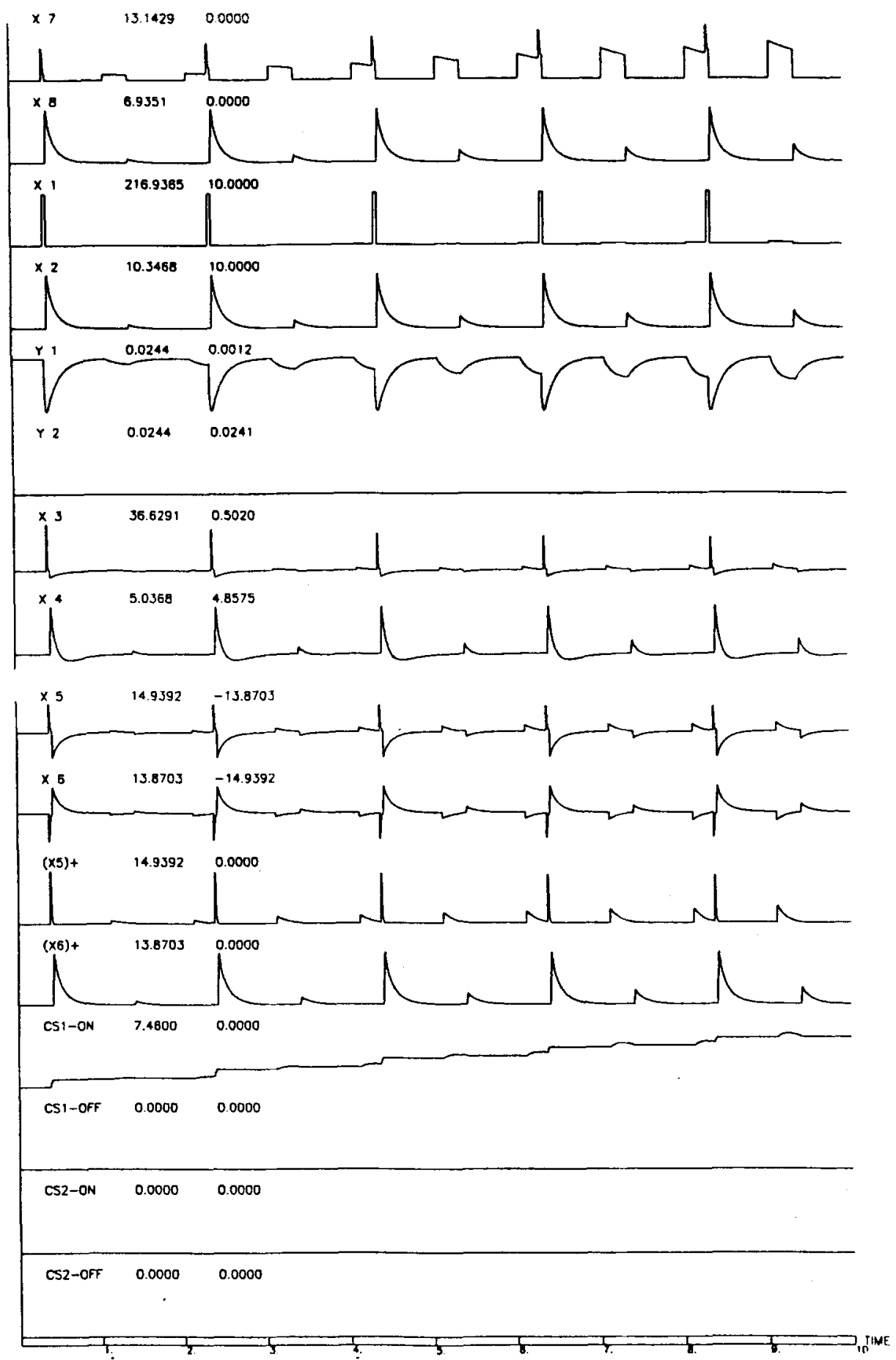

Figure 26. Computer simulation of partial reinforcement with slow habituation and small feedback in a READ I circuit. The conditioned stimulus $\left(\mathrm{CS}_{1}\right)$ is alternately presented with the unconditioned stimulus (US) and without the US during 10 simulated trials. The parameters are those given in Figure 22.

The experimental paradigm for training a conditioned inhibitor is similar to the blocking paradigm (Kamin, $1968,1969)$. In a blocking paradigm, the $\mathrm{CS}_{1}$ may be paired with a shock US. Then the $\mathrm{CS}_{1}$ and $\mathrm{CS}_{2}$ are simultaneously presented, but are also followed by a shock US. The key question in blocking concerns why the $\mathrm{CS}_{2}$ does not become, at least asymptotically, a conditioned excitor. How does prior fear conditioning of the CS " "block" subsequent fear conditioning of the $\mathrm{CS}_{2}$ ?

The blocking paradigm and the conditioned inhibition paradigm thus differ primarily in terms of the consequences of $\mathrm{CS}_{1}+\mathrm{CS}_{2}$ presentations. In blocking, the con- 
sequence is a definite US event. In conditioned inhibition, it is the nonoccurrence of the expected US event. There exists a continuum of other experimental possibilities in which the compound stimulus $\mathrm{CS}_{1}+\mathrm{CS}_{2}$ may be followed by a US that differs from the original US, for example, in its intensity. Then conditioning of the $\mathrm{CS}_{2}$ may undergo unblocking. From this perspective, properties of conditioned inhibition may be interpreted as a limiting case of unblocking properties. ${ }^{2}$

Herein we join the computer simulations of READ circuit dynamics that are described in Part I to the additional cognitive-emotional mechanisms schematized in Figures 1 and 2 to provide a unified real-time explanation of key data about conditioned inhibition. In particular, this explanation clarifies how, despite their similarity, blocking and conditioned inhibition paradigms generate such different behavioral properties.

\section{Conditioned Inhibition as a "Slave" Process}

Our analysis takes as its point of departure the seminal experiments, modeling concepts, and general data discussions provided by Lysle and Fowler (1985) and Miller and Schachtman (1985). Many related experiments will be clarified along the way.

One motivation for Lysle and Fowler's (1985) experiments was the fact that several predictions of the Rescorla and Wagner (1972) model failed to be experimentally confirmed in later studies. In particular, a conditioned inhibitor $\mathrm{CS}_{2}$ does not extinguish when it is presented alone, unlike a conditioned excitor (DeVito, 1980; Owren \& Kaplan, 1981; Witcher, 1978; Zimmer-Hart \& Rescorla, 1974). In addition, a neutral stimulus presented with a conditioned inhibitor $\mathrm{CS}_{2}$ does not acquire excitatory value (Baker, 1974). The experiments Lysle and Fowler (1985) designed to further probe these properties led them to conclude that conditioned inhibition is a "slave" process to conditioned excitation. This concept was experimentally defined and tested using the following general type of paradigm, whose many controls will not be reviewed here.

First, a CS, was paired with a shock US. Next, a compound stimulus $\mathrm{CS}_{1}+\mathrm{CS}_{2}$ was followed by a no-shock interval. Then a number of different manipulations were carried out on several different groups of animals. In one group of animals (denoted by $\mathrm{CS}_{1}\left[\mathrm{CS}_{2}\right]$ ), the $\mathrm{CS}_{1}$ was extinguished by being followed by a no-shock interval. In another group of animals (denoted by $\mathrm{X}\left[\mathrm{CS}_{2}\right]$ ), the training context $\mathrm{X}$ was extinguished by being followed by a no-shock interval. In a third group of animals (denoted by $\mathrm{N}\left[\mathrm{CS}_{2}\right]$ ), neither $\mathrm{CS}_{1}$ nor $\mathrm{X}$ was extinguished. Then a retardation test was performed to discover how quickly the conditioned inhibitor $\mathrm{CS}_{2}$ could be trained as a conditioned excitor by being randomly paired with shock on $50 \%$ of its trials. The data showed that conditioned suppression developed least rapidly for the $\mathrm{N}\left[\mathrm{CS}_{2}\right]$ group, more rapidly for the $\mathrm{X}\left[\mathrm{CS}_{2}\right]$ group, and most rapidly for the $\mathrm{CS}_{1}\left[\mathrm{CS}_{2}\right]$ group. Indeed, the $\mathrm{CS}_{1}\left[\mathrm{CS}_{2}\right]$ group developed suppression almost as rapidly as comparison groups that were tested using a novel CS rather than $\mathrm{CS}_{2}$. In other words, prior extinction of the conditioned excitor $\mathrm{CS}_{1}$ seemed to deactivate the conditioned inhibitory properties of $\mathrm{CS}_{2}$ in the subsequent retardation test, as did, to a lesser extent, extinction of the context $X$, which had also acquired properties of a conditioned excitor.

To further analyze these properties, Lysle and Fowler (1985) tested whether fear of the $\mathrm{CS}_{2}$ or the US was in the test context or in the animal. In the latter case, "a nonassociative mechanism could be postulated whereby inhibition is motivated and thus maintained by an excitatory representation of generic form" (Lysle \& Fowler, 1985, p. 83). To this end they performed experiments that demonstrated that if, in conjunction with extinction of the conditioned excitor $\mathrm{CS}_{1}$,

the animal receives presentations in a different context of the US by itself, for a novel CS, or correlated either positively or negatively with $\left[\mathrm{CS}_{1}\right]$, then the inhibitory property of $\left[\mathrm{CS}_{2}\right]$ will be maintained without loss. . . Furthermore, if, following extinction in the original context, the animal receives US presentations for the same or a different CS in that context, then the inhibitory property of $\left[\mathrm{CS}_{2}\right]$ will be restored apparently to full strength. (Lysle \& Fowler, 1985, p. 90)

The remarkable aspect of these results is that such flexible relationships between the extinction and retraining of conditioned excitatory events and a conditioned inhibitor can have such dramatic effects upon how and whether the conditioned inhibitory property manifests itself in a test context.

The fact that extinction of the conditioned excitor $\mathrm{CS}_{1}$ deactivates the conditioned inhibitory properties of $\mathrm{CS}_{2}$, but reconditioning in another context reinstates $\mathrm{CS}_{2}$ as a conditioned inhibitor, prompted Lysle and Fowler (1985) to propose that conditioned inhibition is a "slave" process to conditioned excitation.

\section{Conditioned Inhibition as a "Comparator" Process}

According to Rescorla (1968), excitatory conditioning is obtained whenever $P(\mathrm{US} / \mathrm{CS})>P(\mathrm{US} / \overline{\mathrm{CS}})$, inhibitory conditioning when $P(\mathrm{US} / \mathrm{CS})<P(\mathrm{US} / \overline{\mathrm{CS}})$, and no conditioning when $P(\mathrm{US} / \mathrm{CS})=P(\mathrm{US} / \overline{\mathrm{CS}})$, where $\overline{\mathrm{CS}}$ denotes "no CS."

In a more recent exploration of contingency effects on classical conditioning, Miller and Schachtman (1985) further analyzed these properties by paying particular attention to how the context $\mathrm{X}$ may become conditioned to the US, and the effects of such associations on conditioned inhibition and excitation. They therefore controlled both the probability $P(\mathrm{US} / \mathrm{CS})$ and the probability $P(\mathrm{US} / \overline{\mathrm{CS}})$. They noted that if $P(\mathrm{US} / \mathrm{CS})=.33$ and $P(\mathrm{US} / \overline{\mathrm{CS}})=0$, then the CS became a conditioned excitor. In contrast, if $P(\mathrm{US} / \mathrm{CS})=.33$ but $P(\mathrm{US} / \overline{\mathrm{CS}})=.67$, then the CS became a conditioned inhibitor. Thus, knowing $P(\mathrm{US} / \mathrm{CS})$ alone is not sufficient to predict the excitatory or inhibitory properties of the CS. The relevance of context-US associations was vividly raised by this manipulation, since during $P(\mathrm{US} / \overline{\mathrm{CS}})$ trials, no CS occurred. 
Miller and Schachtman (1985) explored the role of context-US associations and showed that the "critical factor was whether or not unsignaled shocks were given in the conditioning context" (p. 60). They did this by training two experimental groups $[P(\mathrm{US} / \mathrm{CS})=.33$, $P(\mathrm{US} / \overline{\mathrm{CS}})=.67$ and $P(\mathrm{US} / \mathrm{CS})=.33, P(\mathrm{US} / \overline{\mathrm{CS}})=0$ ] in an experimental context $A$. They then broke up each group into two test groups, and tested one group's reaction to the CS in context A and the other group's reaction to the CS in a novel context $\mathrm{B}$. Both groups that were trained on $P(\mathrm{US} / \mathrm{CS})=.33, P(\mathrm{US} / \overline{\mathrm{CS}})=.67$ showed short-latency responses to the $C S$, whereas both groups trained on $P(\mathrm{US} / \mathrm{CS})=.33, P(\mathrm{US} / \overline{\mathrm{CS}})=0$ showed longlatency responses to the CS. Thus, if the CS was trained as a conditioned excitor, it preserved this property in either the training context or a novel context. The same was true if the CS was trained to be a conditioned inhibitor. The latter result is of particular interest, since it demonstrated that a conditioned inhibitor could maintain its inhibitory property in a novel context $B$ in which no excitatory conditioning had taken place. In particular, the inhibitory effect of the CS in this situation could not be due to the unmasking in context B of a weaker excitatory effect than that which was conditioned to the training context $A$.

After conducting a variation of this paradigm, Miller and Schachtman (1985) concluded that "the training location as opposed to the test location plays the role of the associative comparator in determining whether or not a CS will be an effective conditioned inhibitor or conditioned excitor" (p. 61). To show this, they again chose $P(\mathrm{US} / \mathrm{CS})=.33$ with $\mathrm{CS}$ conditioning occurring in context A; this time, however, no unsignaled shocks were delivered in context $A$, and for half the animals the probability of unsignaled shock in context $B$ was .67. Testing of the CS in either context A or context B showed that it was a source of conditioned excitation, independent of the occurrence of signaled shocks in context $B$ and, as in the past experiment, independent of which context was used for testing.

An interesting finer point in these results was that the total conditioned excitatory effect of the CS was not significantly increased by testing it in the fearful context $B$. This result is consistent with the property of associative averaging, rather than associative summation, of the total amount of conditioned fear (Section 11).

Miller and Schachtman (1985) realized that different temporal contingencies were imposed by training and testing in the same or different contexts. They particularly noted data of Kleiman and Fowler (1984) wherein

unsignaled shocks delivered in moderately close temporal proximity to a nonreinforced stimulus (but not so close as to produce excitatory conditioning) will increase the effective inhibitory strength of this stimulus relative to that produced by unsignaled shocks delivered in the middle of the intervals between presentations of the target stimulus.

(Miller \& Schachtman, 1985, p. 63)

We will trace this temporal effect to whether conditioning occurs to a directly activated on-channel or to an in- directly activated antagonistic rebound within an offchannel.

Miller and Schachtman (1985) discovered an important asymmetry in the processing of a conditioned inhibitor when they "asked whether the comparator baseline was the excitatory value of the conditioning context at the time of conditioning or at the time of testing' (p. 64). To test this, they inflated or deflated the associative value of the context during the retention interval before the test began. For example, after training on $P(\mathrm{US} / \mathrm{CS})=.33$, $P(\mathrm{US} / \overline{\mathrm{CS}})=.67$, they deflated the context in one group by extinguishing it on $P(\mathrm{US} / \overline{\mathrm{CS}})=0$. Then the CS was tested in both the deflated group and a nondeflated group. The CS elicited a longer lick latency after context deflation than in the absence of context deflation. This is a remarkable result, because it was accomplished without presenting either the CS or the US in the $P(\mathrm{US} / \overline{\mathrm{CS}})=0$ situation. Miller and Schachtman concluded that

the comparator baseline is the current associative value of the conditioning context rather than the associative value of the conditioning context at the time of conditioning.... The critical comparison does not occur until the time of testing. Thus the information retained over the retention interval is apparently the independent associative strengths of the CS, i.e., $P(\mathrm{US} / \mathrm{CS})$, and the conditioning context, i.e., $P$ (US/ $\overline{\mathrm{CS}})$, rather than solely the outcome of the comparison, i.e., $P(\mathrm{CR} / \mathrm{CS})$. (p. 65)

We will show how a combination of an adaptation level shift, associative averaging, and antagonistic rebound properties can explain this result, in much the same way that it can explain the finding of Bottjer (1982) that a novel stimulus presented just before the CS can restore the inhibitory power of the CS.

In contrast to their results on contextual deflation, Miller and Schachtman (1985) also demonstrated that "no amount of contextual inflation will affect the comparator role of the conditioning context"' (p. 66). Both the effect of contextual deflation and the non-effect of contextual deflation will be explained using the same mechanisms, as will the fact that US-alone presentations do degrade CS responding either before (Holman, 1976) or during CS conditioning, but not after (Jenkins \& Lambos, 1983). The present theory thus has a broader explanatory range than does the comparator hypothesis; Miller and Schachtman (1985) remarked that they "currently do not have any reasonable hypothesis as to why contextual inflation [CI] appears to be ineffective, whereas contextual deflation does influence responding ... it remains unclear why unsignaled US's following conditioning apparently fail to augment $\mathrm{CI}$, whereas US's during conditioning do provide CI" (p. 67).

In addition, we suggest explanations of other important data that Miller and Schachtman (1985, p. 69) summarized, such as "why a conditioned inhibitor attenuates excitor behavior on a summation test far more than does a novel stimulus (Pavlov, 1927)" and why, as in the experiments of Cotton, Goodall, and Mackintosh (1982) an " $\mathrm{A} \rightarrow$ large shock $\mathrm{AX} \rightarrow$ small shock procedure renders 
$X$ effectively inhibitory as measured in a summation test with a previously conditioned excitor (B) when B had previously been paired with a large shock, but not when B had been paired with a small shock' (Miller \& Schachtman, 1985 , p. 69). The same mechanisms also clarify why, although conditioned excitation is retarded, conditioned inhibition is facilitated by preconditioning US-alone exposures (Hinson, 1982); why $\mathrm{A}+/ \mathrm{X}$ - trials may produce some conditioned inhibition, but only weakly if at all; and why pretest extinction of A restores responding to $\mathrm{X}$ in an overshadowing paradigm (Kaufman \& Bolles, 1981). These results also extend beyond the reach of the comparator hypothesis, inasmuch as "the comparator hypothesis ... is silent concerning how either the nominal CS or context accrue excitatory associative strength" (Miller \& Schachtman, 1985, p. 81).

\section{A Theoretical Review:}

The Synchronization and Peristence Problems of Pavlovian Conditioning

Our explanation of these demanding data about conditioned inhibition is a variant of an explanation of blocking in terms of the following types of processes (Grossberg, 1975, 1982a; Grossberg \& Levine, in press): How does the pairing of a $\mathrm{CS}_{1}$ with a US in the first phase of a blocking experiment endow the $\mathrm{CS}_{1}$ cue with properties of a conditioned, or secondary, reinforcer? How do the reinforcing properties of a cue, whether primary (US) or secondary $\left(\mathrm{CS}_{1}\right)$, shift the focus of attention toward its own processing? How does the limited capacity of attentional resources arise, so that a shift of attention toward one set of cues $\left(\mathrm{CS}_{1}\right.$ or US) can prevent other cues from being attended $\left(\mathrm{CS}_{2}\right.$ or $\left.\mathrm{CS}\right)$ ? How does withdrawal of attention from a cue prevent that cue from entering into new conditioned relationships?

Mechanisms to instantiate these processes have been derived from solutions of several simple, but basic, neural design problems, which came into view through real-time analyses of conditioning data.

The first design problem, called the synchronization problem, was posed in Grossberg (1971, pp. 227-237). This problem asks how CS-US associations can develop in a stable fashion in spite of the variability of the time lag between CS and US. The synchronization problem came into focus as a result of quantitative results from previous work (Grossberg, 1969c, 1970), which showed that each elementary sensory representation or motor command could be represented mathematically as a spatial pattern of activation across a network of cell populations. If activity at a population coding a CS was followed repeatedly by the same US, the LTM traces activated by the CS population could cumulatively learn the spatial pattern corresponding to that US. However, if the CS was followed at different time intervals by two or more events, among which only a single US occurred, the CS-activated LTM traces would not learn the spatial pattern corresponding to the US. Instead, they would learn a mixture of the spatial patterns corresponding to all the events that occurred when the CS was active, whether meaningful to the organism or not. Such a mixture would typically encode little useful information about the environment and would certainly not resemble the US pattern.

The synchronization problem hereby brought into focus two related problems of fundamental importance: How does an organism know how to distinguish significant events for encoding in LTM among all the irrelevant environmental fluctuations that never cease to occur? How are conditioning systems designed to be capable of stably operating in continuous, or real, time, despite the fact that meaningful events, such as novel events and USs, occur at irregular and discrete time intervals?

Grossberg's (1971) analysis of the synchronization problem led to the proposal that there exist populations of cells, called D for drive representations (Figures $1 \mathrm{~b}$ and 2 ), that are separate from the sensory representations of particular stimuli but are related to particular drives and emotions. Workers such as Bower (1981; Bower, Gilligan, \& Monteiro, 1981) have called such drive representations "emotion nodes." A food US, for example, unconditionally activates the $\mathrm{D}$ population corresponding to the hunger drive if the hunger drive level is sufficiently high. Repeated pairing of a CS with a food US thus causes pairing of stimulation of the CS sensory representation, which we denote by $S_{C S}$, with that of the D representation of the hunger drive, which we denote by $D_{H}$. If the $S_{C S} \rightarrow D_{H}$ synapses are assumed to be modifiable according to an associative rule, such as Equation 14, then the pairing $S_{C S} \rightarrow D_{H}$ can become strengthened, so that eventually the CS by itself becomes able to activate the drive representation $\mathrm{D}_{\mathbf{H}}$ and thereby becomes a conditioned reinforcer for food. Once a neutral CS (call it $\mathrm{CS}_{1}$ ) has been conditioned, it can be used as a US to reinforce responses to another $\mathrm{CS}$ (call it $\mathrm{CS}_{2}$ ) in a later experiment. Once the $\mathrm{S}_{\mathrm{CS}_{\mathbf{1}}} \rightarrow \mathrm{D}_{\mathrm{H}}$ synapses have been strengthened, repeated presentation of $\mathrm{CS}_{2}$ followed by $\mathrm{CS}_{1}$ can, in turn, strengthen the associative $\mathrm{S}_{\mathrm{CS}_{2}} \rightarrow \mathrm{D}_{\mathrm{H}}$ synapses, as in Figure 15.

Pathways D $\rightarrow \mathrm{S}$ from the drive representations to the sensory representations were also derived and shown to be conditionable. Conditioning in the $D \rightarrow S$ pathways was related to classical concepts about incentive motivation and shown to overcome some serious problems involving heuristic approaches to the definition of motivation. Whereas reinforcement acts directly upon the efficacy of $S \rightarrow D$ pathways in this model, the entire conditionable pathway $S \rightarrow D \rightarrow S$ regulates motivational support for the learning and performance of conditioned responses along stimulus-response ( $\rightarrow R$ ) circuits (Figure 2).

In order to relate $S \rightarrow D$ conditioning, $D \rightarrow S$ conditioning, and $S \rightarrow R$ conditioning, and thereby to analyze how the $S \rightarrow D \rightarrow S$ feedback loop regulates attention toward motivationally salient cues, Grossberg (1975; reprinted in Grossberg, 1982c) defined and analyzed the persistence problem of classical conditioning, also known as the turkey-love fiasco. This problem arose from consideration of another typical conditioning situation which 
seems problematic only when one attempts to build a realtime model.

In Figure 27b, the cues $\mathrm{CS}_{1}$ and $\mathrm{CS}_{2}$ have previously been conditioned to responses $C_{1}$ and $C_{2}$. Responses $C_{1}$ and $C R_{2}$ are assumed to be motivationally incompatible, as are, for example, eating and sex. A catastrophic problem could occur in an improperly designed learning circuit if $\mathrm{CS}_{1}$ and $\mathrm{CS}_{2}$ were then alternately scanned in rapid succession. If only one of the cues had previously been conditioned to a response, then no difficulty would occur (Figure 27a). However, if both cues were already conditioned and if classical conditioning were merely a feedforward process that associatively linked cues with simultaneously active responses, then cross-conditioning from $\mathrm{CS}_{1}$ to $\mathrm{CR}_{2}$ and from $\mathrm{CS}_{2}$ to $\mathrm{CR}_{1}$ could rapidly occur (Figure 27b). This example identifies the core issue: When many cues are processed in parallel, and some of the cues are already conditioned to motivationally incompatible responses, then why are these associations not quickly degraded by cross-conditioning? How can the ubiquity of parallel cue processing be reconciled with the persistence of learned meanings?

The persistence problem was also called the "turkeylove fiasco" to emphasize its basic nature and the absurd world to which it could lead if not actively prevented. During an otherwise uneventful turkey dinner with one's lover, suppose that one alternately looks at lover and tur- (a)

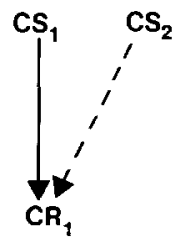

(b)

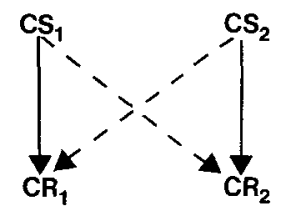

(c)

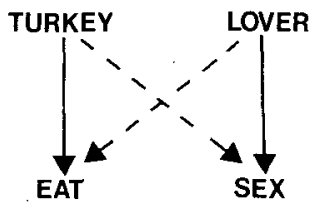

Figure 27. The persistence problem of classical conditioning. (a) $A$ conditioned stimulus $\left(\mathrm{CS}_{j}\right)$ can be quickly associated with the conditioned response $\left(C_{i}\right)$ of a distinct $\mathrm{CS}_{i}$. (b) When each of the conditioned stimuli $\left(\mathrm{CS}_{1}\right.$ and $\left.\mathrm{CS}_{2}\right)$ is already conditioned to a distinct conditioned response (CR ${ }_{1}$ and $\mathrm{CR}_{2}$, respectively) at the beginning of an experiment, alternate scanning of $\mathrm{CS}_{\mathbf{1}}$ and $\mathrm{CS}_{\mathbf{2}}$ does not always cause rapid cross conditioning of $C_{1}$ to $C R_{2}$ and $C S_{2}$ to $C R_{1}$, as is made clear in (c), which depicts the absurd consequence that would arise after dining with one's lover. key. Visual cues of one's lover are associated with sexual responses (among others!) and visual cues of turkey are associated with eating responses. Why do we not come away from dinner with tendencies to eat our lover and to have sex with turkeys? The fact that we do not illustrates that the persistence of learned meanings can endure despite the fact that sensory cues that are processed in parallel often control motivationally incompatible responses.

At least two types of mechanisms have been proposed to deal with this fundamental problem: (1) prewired, or innate, connections among preferred sets of internal representations, and (2) dynamic regulation of conditioned associations via attentional mechanisms. In general, a combination of both types of factors may be operative, since the non-equipotentiality of prewired connections can facilitate conditioning among certain sets of events above others.

Seligman illustrated the role of non-equipotentiality during an experience when he

felt the effects of the stomach flu six hours after eating filet mignon with sauce Bearnaise. The next time I had sauce Bearnaise, I could not bear the taste of it... . Neither the filet mignon, nor the white plates off which I ate the sauce, nor Tristan und Isolde, ... nor my wife . . . , became aversive. (Seligman \& Hager, 1972)

Several experiments also suggest that some combinations of stimuli and reinforcers result in faster conditioning than do others. For instance, Garcia and Koelling (1966) found that when a compound gustatory and auditory stimulus are paired with agents that produce nausea, gustatory but not auditory stimuli are associated with nausea. On the other hand, when the compound stimulus is paired with a shock US, the auditory stimulus is associated with the US (Domjan and Wilson, 1972). Foree and LoLordo (1973) showed that pigeons associate visual stimuli with a food US more readily than auditory stimuli with a food US, and that this relationship is reversed when a shock US is used.

Seligman and Hager (1972) suggested that the results could be explained in terms of a selective associative difference by which a given CS is innately more "prepared" to be associated with a given US than with others. Mackintosh (1973) proposed that previous experience with the difference in the correlation of different classes of CSs and USs determines their predisposition to be associated at a later time.

Grossberg (1975) suggested a mechanistic solution of the persistence problem in which the possible nonequipotentiality of innate connections was acknowledged, but additional attentional regulatory mechanisms were invoked to deal with the case in which the sensory CSs were approximately equipotential with respect to pairs of motivationally incompatible responses. This solution proposed how incentive motivational feedback due to conditionable $D \rightarrow S$ pathways could shift an organism's attentional focus to preferentially process previously experienced 
motivationally compatible cues. Thus attention-switching between sets of motivationally compatible cues can dynamically buffer motivationally incompatible sets of cues against rapid cross-conditioning, since when a sensory representation $S$ has a zero signal $S_{k}$ in Equations 14 and 15 , no new conditioning of its LTM traces $z_{k 7}$ and $z_{k 8}$ can occur. In addition, a sensory cue that possesses a large conditioned $\mathrm{S} \rightarrow \mathrm{D} \rightarrow \mathrm{S}$ feedback pathway can quickly prime the STM of motivationally compatible sensory cues while amplifying the STM activity of its own sensory representation (Figure 2). Reinforcing cues can hereby draw attention to themselves and to their entire motivational "set" via self-generated incentive motivational feedback signals (Figure 28). Killeen (1982a, 1982b) also emphasized the importance of incentive motivation for the modeling of instrumental conditioning data.

\section{Competition for Limited-Capacity}

\section{Short-Term Memory Activity and Attention}

The sensory representations that emit conditioned reinforcer signals and receive incentive motivational signals also compete among themselves for a limited-capacity STM resource (Figure 2). The ubiquitous occurrence of limited-capacity STM was traced in Grossberg (1973, 1980) to a more basic processing requirement: the ability of cell networks to process spatially distributed input patterns without irreparably distorting these input patterns

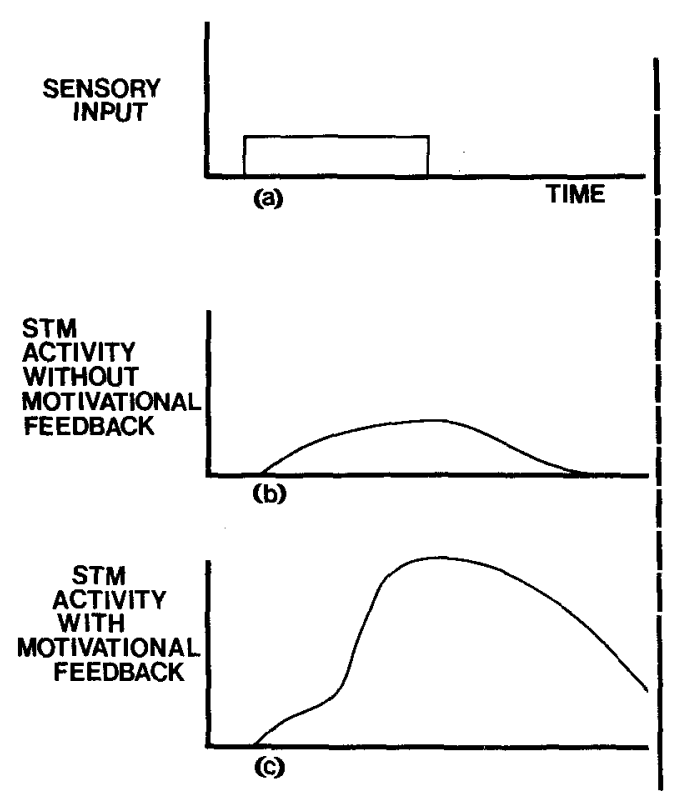

Figure 28. Augmentation of short-term memory (STM) activation at a sensory representation, $S$ cs, by feedback signaling through the pathway $S_{C s} \rightarrow D \rightarrow S_{C s}$. In response to the sensory input (a) received by $S_{C S}$, the $S T M$ activation profile before learning is as schematized in (b). After learning within the $S_{c s} \rightarrow D \rightarrow S_{c s}$ pathway takes place, the initial activation remains as in (b). However, as the feedback signals are registered, the STM activation of Scs can be greatly amplified and prolonged, as schematized in (c). due to either internal noise or saturation effects. This noise-saturation dilemma can be prevented by an on-center off-surround anatomy through which the cells interact via mass action (or shunting) laws. Such a network interaction implies, without further assumptions, that the total suprathreshold activation of the network tends to be conserved, and thus possesses a limited capacity.

When such a network is also designed to accomplish STM storage, its on-center off-surround interactions are recurrent, or feedback, interactions in which the cells excite themselves and inhibit other cells via feedback pathways (Figure 2). In addition to its noise-saturation and limited-capacity properties, such a recurrent on-center offsurround network contrast enhances an input pattern before storing the contrast-enhanced activation pattern that emerges across the cells in STM (also called working memory). Thus one must distinguish between the input pattern and the more focal STM activity pattern that it generates. Attention is paid to those sensory representations whose cells receive a positive level of stored STM activity.

When incentive motivational feedback signals form part of the total input pattern to the sensory representations (Figure 2), these signals can bias the competition for STM activity toward motivationally salient cues. Due to the limited capacity of STM, motivationally salient cues, in particular primary and secondary reinforcers, can draw attention to themselves via their strong conditioned $\mathrm{S} \rightarrow \mathrm{D} \rightarrow \mathrm{S}$ feedback loops. In order to initiate such an attention shift, such cues must first start to be processed due to their sensory properties. After sensory processing is initiated, it can activate the learned reinforcing and motivating pathways of the cues, and can thereby help to direct the ultimate allocation of sensory and attentional resources.

Once attention shifts away from a sensory representation, its activity can become small or even subthreshold. If, by whatever means, an attention shift causes a signal $S_{k}$ from a sensory representation to become small or zero in the LTM equations 14 and 15, then the LTM traces of the representation learn very slowly or not at all.

\section{Gated Dipoles and Opponent Extinction}

Gated dipoles were originally derived in Grossberg (1972b) to explain how the offset of a reinforcer of positive (or negative) sign can generate an antagonistic rebound to which a simultaneous CS can be conditioned as a reinforcer of negative (or positive) sign. Using these gated dipoles, the drive representations in Figure 2 were organized into the on-channels and off-channels of recurrent gated dipoles. These gated-dipole circuits were, in turn, linked together via competitive interactions into gated-dipole fields, which were designed to choose that drive representation whose combination of sensory, reinforcing, and homeostatic constraints was most favorable at any given time ("winner-take-all"). The chosen chan- 
nel could release incentive motivational output signals to the sensory representations and thereby focus attention upon the motivationally most favored sensory representations. These enhanced representations could thereupon generate output signals to release conditioned responses consistent with these momentary sensory and motivational constraints.

A surprising property of a gated dipole is that the unexpected nonoccurrence of an event can trigger an antagonistic rebound by causing a sudden increment in its nonspecific arousal level $I$ (Grossberg, 1972b, 1987a). These two types of rebound-inducing events clarified how a large on-conditioned reinforcer value of CS-that is, its on-LTM trace $z_{k 7}$ in Equation 14-could be extinguished by conditioning its off-LTM trace $z_{k 8}$ in Equation 15 . Such off-conditioning of $z_{k 8}$ may be due to reinforcing inputs delivered directly to the dipole's off-channel. Off-conditioning may also be due to antagonistic rebounds at the off-channel in response to either sudden offsets of on-channel inputs or unexpected nonoccurrence of on-channel reinforcers. This multiplicity of conditions leading to off-conditioning has previously been used to clarify many paradoxical properties of conditioning and extinction data, and will also play an important explanatory role herein.

A new property of extinction has been understood through the quantitative analysis of a READ circuit described in Part $\mathrm{I}$. This property is called opponent extinction. Opponent extinction clarifies how a conditioning circuit in which passive extinction does not occur can prevent its LTM traces from saturating at maximal values due to a progressive accumulation of associative strength over many conditioning trials. Opponent extinction also shows how associative memories may be actively extinguished even if they do not passively extinguish. The opponent extinction property is based upon the dissociation of associative read-in and read-out that was related to conditioning at dendritic spines in Section 13. Opponent extinction occurs as follows.

If, by any means, off-conditioning proceeds until $z_{k 8} \cong z_{k 7}>0$, then the conditioned reinforcer signals $S_{k} z_{k} 7$ and $S_{k} z_{k 8}$ of a conditioned reinforcer to a READ circuit become approximately equal. This circumstance can actively extinguish the LTM traces $z_{k 7}$ and $z_{k 8}$ as follows. Suppose for definiteness that only signal $S_{k}$ is positive at any time. Then the difference signal $\Delta \cong 0$ in Equation 34 . Consequently $x_{5} \cong 0 \cong x_{6}$, by Equations 21 and 22 . Thus both $z_{k 7}$ and $z_{k 8}$ approach zero, by Equations 14 and 15 . In summary, as the on-LTM trace and off-LTM trace of a conditioned reinforcer become approximately equal, these LTM traces are actively extinguished, due to the fact that conditioned reinforcer learning tracks the net imbalance of activation across the dipole's on-channel and off-channel. This is the opponent extinction process. More generally, conditioned reinforcer LTM traces continually readjust themselves to track the net imbalance they detect in all the environmental contexts within which they are activated. Opponent extinction hereby avoids the possible saturation at maximal values of both LTM traces $z_{k}$ ? and $z_{k 8}$, no matter how many experiments activate $S_{k}$.

\section{Adaptive Resonance Theory: Expectation, Mismatch, Reset, and Rebound}

The gated dipole's rebound properties emphasize that cognitive, notably unexpected, events play a critical role in the modulation of reinforcement, conditioning, and extinction processes. The conceptual and data analyses generated by this cognitive-emotional connection led directly to the discovery and development of adaptive resonance theory (Grossberg, 1976a, 1976b, 1978, 1980; reprinted in Grossberg, 1982c). Adaptive resonance theory has, by now, been used to analyze and predict a large interdisciplinary data base and has undergone substantial technical development (Carpenter \& Grossberg, 1987; Cohen \& Grossberg, 1987; Grossberg, 1987a, 1987b). Only those qualitative features of the theory that are needed to explain data about conditioned inhibition will be summarized herein.

Adaptive resonance theory suggests how internal representations of sensory events, including CSs or USs, can be learned in a stable fashion despite the potentially erosive effects of irrelevant environmental fluctuations. Among the mechanisms for the stable self-organization of sensory recognition codes is the read-out of learned top-down expectations that are matched against bottomup sensory signals (Figure 1). When a mismatch occurs, a nonspecific arousal burst is triggered via an orienting subsystem. This arousal burst acts to reset the sensory representations of all cues that are currently being stored in STM (Figure 29). In particular, representations with high STM activation tend to become less active, representations with low STM activation tend to become more active, and the novel event that caused the mismatch tends to be more actively stored than it would be have been had it been expected (Grossberg, 1982a, 1987a). These properties can be traced to the combined action of gated-dipole interactions and limited-capacity competitive interactions that are hypothesized to take place among the sensory representations. As a result of such an STM-reset event, sensory representations that had been actively reading out an erroneous expectation become less active in STM; hence, the expectation can be updated. Representations that were attentionally blocked before the reset event occurred can become unblocked, or dishabituated, in STM; they code sensory information that may have been erroneously unattended. Finally, the novel event that triggered the mismatch becomes more active in STM; it encodes potentially important new information.

In order to deal effectively with temporal order effects, the architecture schematized in Figure 1 must be augmented by mechanisms for storage of event sequences in working memory (Grossberg \& Stone, 1986a, 1986b) and for the learning of temporal discriminations (Grossberg \& Schmajuk, 1987). 


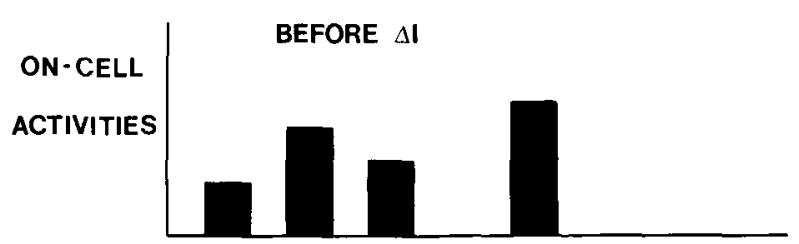

ON-CELL POPULATIONS

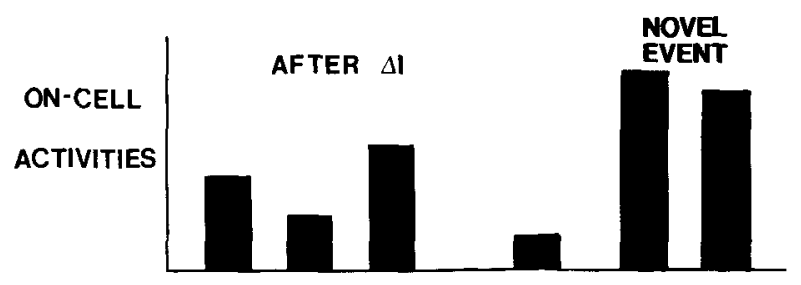

ON-CELL POPULATIONS

Figure 29. Short-term memory reaction to an arousal-mediated $(\Delta I)$ unexpected event. The arousal burst $\Delta I$ tends to inhibit, or reset, populations that were very active before the expected event and to enhance, or dishabituate, populations that were weakly active before the unexpected event. The novel event that triggered $\Delta I$ is also preferentially stored. Inactive populations remain inactive, but they are sensitized by a gain change. This type of global reset event gives more short-term memory activity to those populations that did not control the actions leading to the unexpected outcome, including cells that code the unexpected outcome.

\section{Parallel Learning of Sensory Expectation and Conditioned Reinforcement}

The synthesis of adaptive resonance mechanisms with conditioning mechanisms shows that the internal representation of a sensory event controls at least two distinct types of output signals: learned top-down expectations within an attentional subsystem (Figure 1a) and learned conditioned reinforcer signals to a READ circuit (Figures $1 \mathrm{~b}$ and 3). The distinct, but interacting, properties of these signals in different learning environments are critical to our explanations of conditioned inhibition data. Notable are the interactions whereby a disconfirmed sensory expectation can cause an antagonistic rebound within a READ circuit.

Suppose, for example, that an active sensory representation of a conditioned reinforcer is reset due to a sensory mismatch with its top-down expectation. The reset event causes a rapid decrease in the STM activity of the sensory representation and, thus, in its output signal $S_{k}$ to the READ circuit in Equations 12 and 13. As a result, its conditioned reinforcer inputs $S_{k} z_{k 7}$ and $S_{k} z_{k 8}$ to the READ circuit also decrease. If the cue is an onconditioned reinforcer (viz., $z_{k 7} \gg z_{k 8}$ ), an antagonistic rebound can hereby be elicited in the READ circuit's offchannel.

Using the above properties, we now provide a unified explanation of data about conditioned inhibition. In particular, we suggest an explanation of why a conditioned excitor extinguishes, yet a conditioned inhibitor does not extinguish. This explanation clarifies how this difference obtains, despite the facts that a given CS could be trained to become either a conditioned excitor or a conditioned inhibitor and that during extinction trials, only the CS is presented. In addition, the explanation shows how the different affective properties of excitors and inhibitors are controlled, and utilizes the difference between conditioning of a CS to an affective reaction (viz., its role as a conditioned reinforcer) and conditioning of the same CS to a predicted sensory event (viz., its role as a source of conditioned expectation).

\section{Conditioning and Extinction of a Conditioned Excitor}

When a $C_{1}$ is paired with shock on successive conditioning trials, several things happen in the model. The sensory representation $S_{1}$ of the $C_{1}$ is conditioned to the drive representation $\mathrm{D}_{f}$ corresponding to a fear reaction, both through its conditioned reinforcer pathway $S_{1} \rightarrow D_{f}$ and through its incentive motivational pathway $D_{f} \rightarrow S_{1}$. As a result, later presentations of $\mathrm{CS}_{\mathbf{1}}$ tend to generate an amplified STM activation of $S_{1}$, and thus $C_{1}$ is preferentially attended. Due to the limited capacity of STM, less salient cues tend to be attentionally blocked when $\mathrm{CS}_{\mathbf{1}}$ is presented.

As the cognitive-emotional feedback loop $S_{1} \rightarrow D_{f} \rightarrow S_{1}$ is strengthened during conditioning trials, $S_{1}$ also learns a sensory expectation of the shock. During extinction, $\mathbf{C S}_{1}$ is presented on unshocked trials. We assume, as in Figures 13-21, that the numerical parameters of the READ circuit are chosen to prevent significant passive decay of LTM traces from occurring; that is, we assume that extinction of conditioned reinforcer learning is due to an active process of counterconditioning, as in Section 24. When the expected shock does not occur, a mismatch occurs with the learned expectation read-out by $S_{1}$. As described in Section 26, the STM activity of $S_{1}$ is quickly reduced and an antagonistic rebound occurs in the READ circuit. This rebound inhibits the fear reaction that is regulated by the on-channel, and activates the relief reaction (Denny, 1970; Dunham, 1971; Dunham, Mariner, \& Adams, 1969; Hammond, 1968; Masterson, 1970; McAllister \& McAllister, 1970; McAllister, McAllister, \& Douglass, 1971; Rescorla, 1969; Rescorla \& LoLordo, 1965; Reynierse \& Rizley, 1970; Weisman \& Litner, 1969) that is regulated by the dipole's off-channel $D_{r}$.

The collapse in $S_{1}$ 's STM activity may be partial or complete. We assume for definiteness that it is partial, and describe in this case how conditioning within the $S_{1} \rightarrow D_{f} \rightarrow S_{1}$ pathway is antagonized by reboundcontingent conditioning, which progressively extinguishes $\mathrm{CS}_{1}$ as a source of conditioned fear.

The collapse of $S_{1}$ 's activity occurs prior to the rebound from $D_{f}$ to $D_{r}$. Due to the hysteretic properties of a feedback competitive circuit, there exists a time interval during which $\mathrm{D}_{f}$ remains active after $S_{1}$ 's activity collapses. During this time interval, the incentive motivational pathway $D_{f} \rightarrow S_{1}$ is weakened due to the same conditioning mechanism (Section 24) that could lead to total extinction were $S_{1}$ to become totally inactive. If $\mathrm{CS}_{1}$ is followed 
by a no-shock interval on successive learning trials, the weakening of the $D_{f} \rightarrow S_{1}$ pathway is cumulative. As a result, on a later presentation of $C S_{1}, D_{f}$ will supply less feedback to $S_{1}$, so that $S_{1}$ will be less attended than previously, but could possibly still be more attended than an irrelevant situational cue.

In addition to conditioned weakening of the $\mathrm{D}_{f} \rightarrow S_{1}$ pathway, the $S_{1} \rightarrow D_{f}$ pathway can be greatly weakened or even totally extinguished. This is because $S_{1}$ remains active after it is reset. Consequently, $S_{1}$ can become associated with an antagonistic rebound at $D_{r}$. This learning may take place at a slower rate than it did when $S_{1}$ was associated with $\mathrm{D}_{f}$, because $S_{1}$ is smaller after reset than before. On the other hand, the maximal possible LTM strength of the $S_{1} \rightarrow D_{r}$ pathway can exceed that of the $\mathbf{S}_{\mathbf{1}} \rightarrow \mathrm{D}_{f}$ pathway, as it does in the simulations summarized in Figures 14 and 15, as well as those in Figures 16 and 17 . If $\mathrm{CS}_{1}$ is followed by a no-shock interval on successive trials, this learning process will be cumulative. Finally a time will occur when the pathway $S_{1} \rightarrow D_{r}$ is as strong as the pathway $S_{1} \rightarrow D_{f}$. As this time is approached, both conditioned reinforcer pathways become extinguished by the mechanism described in Section 24, and $S_{1}$ is extinguished as a conditioned excitor.

In the event that extinction of conditioned reinforcer $S \rightarrow D$ pathways occurs rapidly, it may permit residual $\mathrm{D} \rightarrow \mathrm{S}$ incentive motivational associations to persist. Such residual associations, including the associations which encode sensory expectations (Section 25) help to explain how, during successive acquisitions and extinctions, rate of acquisition and extinction may increase as a result of successive reversals (Davenport, 1969; Gonzalez, Berger, \& Bitterman, 1967; Schmaltz \& Theios, 1972).

\section{Conditioning and Nonextinction of a Conditioned Inhibitor}

Suppose that $\mathrm{CS}_{\mathbf{1}}$ has become a conditioned excitor, thereby learning to strongly activate the $S_{1} \rightarrow D_{f} \rightarrow S_{1}$ pathway as well as a learned expectation of a subsequent shock. Now present the compound stimulus $\mathrm{CS}_{1}+\mathrm{CS}_{2}$ and follow it by a no-shock interval. When $\mathrm{CS}_{1}$ and $\mathrm{CS}_{2}$ are simultaneously presented, $S_{1}$ 's activity is amplified by positive feedback through the strong conditioned $S_{1} \rightarrow D_{f} \rightarrow S_{1}$ pathway (Figure 30). As a result of the limited capacity of STM, the STM activity of $S_{2}$ is inhibited, or blocked. (The novelty of $\mathbf{C S}_{2}$ can partially mitigate this blocking effect.) When the expected shock does not occur, the mismatch with $S_{1}$ 's sensory expectation causes both $S_{1}$ and $S_{2}$ to be reset. As described in Section $25, S_{1}$ 's STM activity decreases while $S_{2}$ 's STM activity increases. Due to $S_{1}$ 's decrease, a rebound occurs at $\mathrm{D}_{r}$. Consequently, the unexpected nonoccurrence of the shock enables $S_{2}$ to become associated with $D_{r}$ in both of the pathways $S_{2} \rightarrow D_{r}$ and $D_{r} \rightarrow S_{2}$. These are the primary cognitive-emotional conditioning events that turn $\mathrm{CS}_{2}$ into a conditioned inhibitor.

If $\mathrm{CS}_{1} \rightarrow$ shock trials and $\mathrm{CS}_{1}+\mathrm{CS}_{2} \rightarrow$ no-shock trials are interspersed, then $\mathrm{CS}_{\mathbf{1}}$ 's status as a conditioned exci- tor can be restored against the extinction that takes place on the no-shock trials, in the manner summarized in Section 27. Intermittent restoration of the conditioned excitor properties of $\mathrm{CS}_{1}$ enables $\mathrm{CS}_{1}$ to motivate the cumulative training of $\mathrm{CS}_{2}$ as a conditioned inhibitor on the intervening no-shock trials.

Why does $\mathrm{CS}_{2}$ not extinguish when it is presented alone, as a conditioned excitor does (DeVito, 1980; Owren \& Kaplan, 1981; Witcher, 1978; Zimmer-Hart \& Rescorla, 1974)? Why does a neutral stimulus presented with a conditioned inhibitor not acquire excitatory value (Baker, 1974)? Simple answers are now available.

When $S_{\mathbf{2}}$ is unblocked by the nonoccurrence of shock, it learns a sensory expectation. This sensory expectation

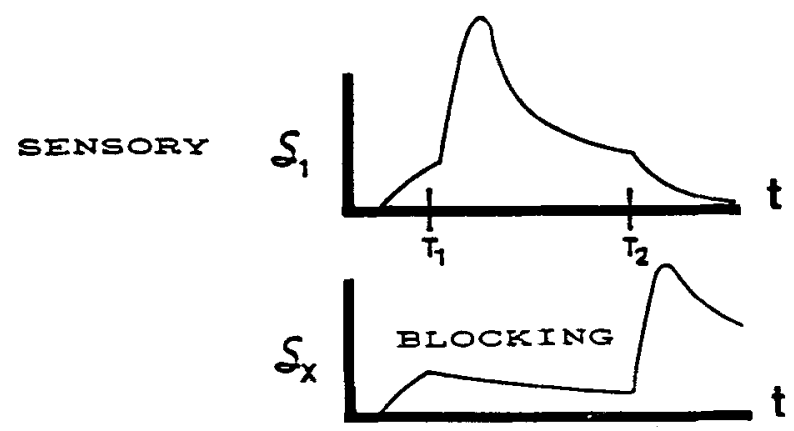

MOTIVATIONAL
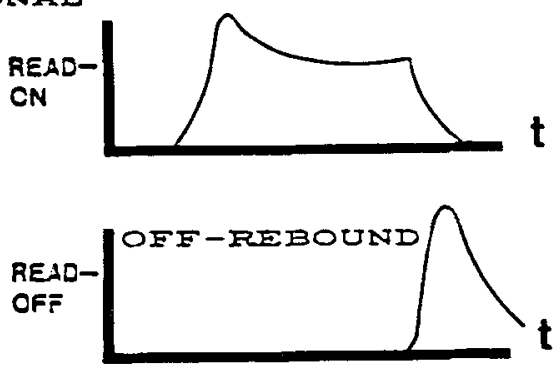

INEUTS

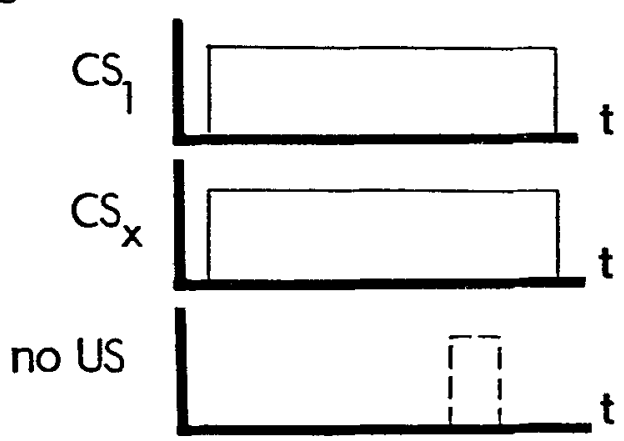

Figure 30. Presentation of conditioned stimuli $\mathrm{CS}_{1}$ and $\mathrm{CS}_{2}$ when $\mathrm{CS}_{1}$ has become a conditioned excitor and the compound stimulus is followed by no-shock. During the no-shock interval between times $T_{1}$ and $T_{2}, S_{1}$ is actively amplified by positive feedback and $S_{2}$ is blocked. During the shock interval, disconfirmation of the expected shock causes both $S_{1}$ and $S_{2}$ to be reset. $S_{1}$ 's short-term memory (STM) activity decreases and $S_{2}$ 's STM activity increases. Due to $S_{1}$ 's increase, $D_{f}$ also decreases, thereby causing a rebound at $D_{r}$. This rebound becomes associated with the increased activity of $S_{2}$. 
does not, however, predict shock. It includes whatever contextual representations are sufficiently salient and repeatable to be cumulatively learned on successive conditioning trials. Thus presentation of the conditioned inhibitor $\mathrm{CS}_{2}$ within a given context does not disconfirm the sensory expectation controlled by $S_{2}$. Since passive extinction does not occur, a conditioned inhibitor $\mathrm{CS}_{2}$ does not extinguish when it is presented alone.

On the other hand, $S_{2}$ does learn to control a strong $S_{2} \rightarrow D_{r} \rightarrow S_{2}$ pathway. Hence it becomes attentionally amplified and can block the processing of neutral stimuli. Thus a neutral stimulus presented with a conditioned inhibitor does not acquire excitatory value (Baker, 1974).

\section{An Explanation of the "Slave" Data}

Our explanation of Lysle and Fowler's (1985) data about conditioned inhibition as a "slave" process combines adaptive resonance properties of expectancy matching and STM reset with READ circuit properties of adaptation level and rebound. The following additional model properties are particularly relevant.

Extinction of a conditioned excitor $\mathrm{CS}_{1}$ includes not only extinction of the feedback pathway $S_{1} \rightarrow D_{f} \rightarrow S_{1}$, but also reconditioning of the sensory expectation associated with $S_{1}$ to anticipate a no-shock, purely contextual, sensory environment. Contextual cues $\mathrm{X}$ can also become conditioned excitors in a situation wherein a conditioned excitor $\mathrm{CS}_{1}$ or a shock occurs at random times. Such contextual cues $\mathrm{X}$ may condition an expectation of shock and a pathway $S_{X} \rightarrow D_{f} \rightarrow S_{X}$ between their sensory representations $S_{\mathrm{X}}$ and the drive representation $\mathrm{D}_{f}$, as does $S_{1}$. The fact that cues $X$ may be attended during no-shock intervals does not, in itself, imply that the $\mathrm{S}_{\mathrm{X}} \rightarrow \mathrm{D}_{f} \rightarrow \mathrm{S}_{\mathrm{X}}$ pathways will extinguish, because extinction does not occur passively (Section 17). Contextual cues extinguish only during time intervals when their expectation of shock is actively disconfirmed, thereby triggering the rebounds that enable $S_{X}$ to become associated with $D_{r}$.

Consider what happens within the model in response to the conditioning experiences of the groups $\mathrm{CS}_{1}\left[\mathrm{CS}_{2}\right]$, $\mathrm{X}\left[\mathrm{CS}_{2}\right]$, and $\mathrm{N}\left[\mathrm{CS}_{2}\right]$ that were defined in Section 20. When $\mathrm{CS}_{1}$ is extinguished by being followed by no-shock intervals, both its sensory expectation of shock and the sensory expectation of shock controlled by attended contextual cues $\mathrm{X}$ are simultaneously extinguished. Likewise, the conditioned reinforcer pathways $S_{1} \rightarrow D_{f} \rightarrow S_{1}$ and $\mathrm{S}_{\mathrm{X}} \rightarrow \mathrm{D}_{f} \rightarrow \mathrm{S}_{\mathrm{X}}$ are simultaneously extinguished. Thus, when $\mathrm{CS}_{\mathbf{2}}$ is trained as a conditioned excitor by being randomly paired with shock on $50 \%$ of its trials, the context in which $\mathrm{CS}_{2}$ occurs is one in which no shock is expected. Thus the occurrence of a shock after $\mathrm{CS}_{2}$ is, in this situation, surprising. In addition, because the fearful effects of $\mathrm{X}$ on $\mathrm{D}_{f}$ have been at least partially extinguished, the net effect of the compound cue $\mathrm{CS}_{2}+\mathrm{X}$ on the gated dipole is to generate a net positive reaction at $D_{r}$. When a shock occurs subsequent to a presentation of $\mathrm{CS}_{2}$, a large mismatch occurs with the sensory expectation of $\mathrm{CS}_{2}$ and
$\mathrm{S}_{2}$ is vigorously reset. Due to the net positive reaction of $D_{r}$ to $C_{2}$, the reset of $S_{2}$ causes a large rebound from $\mathrm{D}_{r}$ to $\mathrm{D}_{f}$. As this rebound from $\mathrm{D}_{r}$ to $\mathrm{D}_{f}$ is forming, the shock itself generates an unconditioned input to $D_{f}$. The rebound and the direct shock input summate to generate an unusually large activation of $D_{f}$. This enhanced fear reaction is learned by $S_{2}$. Thus, we trace the enhanced suppressive effect of $\mathrm{CS}_{2}$ in the $\mathrm{CS}_{1}\left[\mathrm{CS}_{2}\right]$ group to the same types of mechanisms Grossberg (1975) used to explain the partial reinforcement acquisition effect. It is immediately clear from this explanation why extinction of the context $\mathrm{X}$ in group $\mathrm{X}\left[\mathrm{CS}_{2}\right]$ should have a similar, but smaller, effect on the subsequent suppressive effects of $\mathrm{CS}_{2}$.

In contrast, consider what happens in group $\mathrm{N}\left[\mathrm{CS}_{2}\right]$. Here neither $\mathbf{C S}_{1}$ nor $\mathrm{X}$ is extinguished. Thus contextual cues $\mathrm{X}$ are still conditioned to an expectation of shock and to the fear center $\mathrm{D}_{f}$ when retardation testing of $\mathrm{CS}_{2}$ commences. When $\mathrm{CS}_{2}$ is presented in such a context $\mathrm{X}$, an expectation of shock can still be maintained by contextual cues. In addition, although $S_{2}$ is conditioned to $D_{r}$, $\mathrm{X}$ is conditioned to $\mathrm{D}_{f}$. As a result, the net effect of both sets of signals upon the READ circuit is to generate a large adaptation level $\hat{I}$ in Equation 33 and a small, possibly even zero, difference value $\Delta$ in Equation 34 . We consider the case $\Delta=0$ to make our argument in its most extreme form. A similar qualitative argument holds for small values of $\Delta$.

Consider what happens within the model when $\mathrm{CS}_{\mathbf{2}}$ is first followed by a shock. Unlike the case for group $\mathrm{CS}_{1}\left[\mathrm{CS}_{2}\right]$, an expectation of shock does exist. Thus the reset of $S_{2}$ and $X$ will be much less in the $N\left[\mathrm{CS}_{2}\right]$ group than in the $\mathrm{CS}_{1}\left[\mathrm{CS}_{2}\right]$ group. In addition, if $\Delta \cong 0$, this reset event may cause no significant rebound, since both channels of the READ circuit may receive similar conditioned input both before and after the reset event. The primary effect of the shock is to generate an unconditioned input to $D_{f}$. This input does not summate with a rebound from $D_{r}$ to $D_{f}$. Moreover, this unconditioned input is received by a gated dipole with an unusually large adaptation level $\hat{I}$. The net response of a gated dipole to a fixed phasic input is reduced in the presence of a large adaptation level $\hat{I}$ (Grossberg, 1972b, 1987a). Thus conditioning from $S_{2}$ to $\mathrm{D}_{f}$ is much slower in the $\mathrm{N}\left[\mathrm{CS}_{2}\right]$ group than in the $\mathrm{CS}_{1}\left[\mathrm{CS}_{2}\right]$ or $\mathrm{X}\left[\mathrm{CS}_{2}\right]$ groups.

Restoration of the conditioned inhibitory properties of $\mathrm{CS}_{2}$ by presentation of a US is readily explained by the same mechanisms if the US is presented within the same context as the $\mathrm{CS}_{2}$. Such restoration can also be explained if the US is presented in a novel context that shares cues with the original context. These cues may, for example, include similar shapes of the conditioning chambers and similar procedures in the animals' handling.

This explanation of the "slave" properties sets the stage for explaining the "comparator" properties described by Miller and Schachtman (1985) by explicating the mediating role of the context $X$. 


\section{An Explanation of the "Comparator" Data}

How can $\mathrm{CS}_{1}$ become a conditioned excitor if $P\left(\mathrm{US} / \mathrm{CS}_{1}\right)=.33$ and $P\left(\mathrm{US} / \overline{\mathrm{CS}}_{1}\right)=0$, but a conditioned inhibitor if $P\left(\mathrm{US} / \mathrm{CS}_{1}\right)=.33$ and $P\left(\mathrm{US} / \overline{\mathrm{CS}}_{1}\right)=.67$ ? In addition to answering this question, an explanation of the "comparator" properties would also show why there exists a neutral region of values of $P\left(\mathrm{US} / \mathrm{CS}_{1}\right)$ and $P\left(\mathrm{US} / \overline{C S}_{1}\right)$ in which $\mathrm{CS}_{1}$ does not become a conditioned reinforcer. This crossover region often occurs when $P\left(\mathrm{US} / \mathrm{CS}_{1}\right)=P\left(\mathrm{US} / \overline{\mathrm{CS}}_{1}\right)$ (Rescorla, 1968).

In the case $P\left(\mathrm{US} / \mathrm{CS}_{1}\right)=.33, P\left(\mathrm{US} / \overline{\mathrm{CS}}_{1}\right)=0, \mathrm{CS}_{1}$ generates a much stronger conditioned expectation of shock and conditioned attachment to $\mathrm{D}_{f}$ than does $\mathrm{X}$. In the case $P\left(\mathrm{US} / \mathrm{CS}_{1}\right)=.33, P\left(\mathrm{US} / \overline{\mathrm{CS}}_{1}\right)=.67$, the reverse is true. In particular, consider an early learning trial when $\mathrm{CS}_{1}$ is presented under the $P\left(\mathrm{US} / \mathrm{CS}_{1}\right)=.33, P\left(\mathrm{US} / \overline{C S}_{1}\right)$ $=.67$ contingency. Because $S_{X}$ is conditioned more strongly to $D_{f}$ than is $S_{1}, S_{X}$ can partially block $S_{1}$. Suppose on this trial that the shock is unexpectedly absent. When the mismatch occurs, $S_{X}$ can be reset as $S_{1}$ is unblocked. Consequently, $S_{1}$ can be associated with the rebound from $D_{r}$ to $D_{f}$ that is caused by the reset of $X$. This conditioning event tends to make $\mathrm{CS}_{1}$ a source of conditioned relief.

On the other hand, $\mathrm{CS}_{\mathbf{1}}$ is followed by shocks on some trials, because $P(\mathrm{US} / \mathrm{CS})=.33$. Thus the above argument does not necessarily imply that the LTM trace from $S_{1}$ to $D_{f}$ is weaker than that to $D_{r}$. In general, however, the net conditioned fear caused by $S_{1}$ alone is significantly less than the net conditioned fear caused by $S_{X}$ alone, and $S_{1}$ may be a source of net conditioned relief in some parameter ranges. In the case where $S_{1}$ remains conditioned to fear, the ratio $z_{f} / z x_{r}$ is much larger than the ratio $z_{1 f} / z_{1 r}$; that is,

$$
\frac{z \mathrm{x}_{f}}{z_{\mathrm{x} r}}>\frac{z_{1 f}}{z_{1 r}}
$$

where the $z s$ denote the LTM traces in the indicated pathways.

On a later test trial when contextual representations $S_{\mathbf{X}}$ are alone active, they share the limited-capacity STM strength (Equation 35) among themselves. We simplify this constraint by lumping all contextual representations into one and writing

$$
S_{\mathrm{X}}=S \text {. }
$$

During such a time interval, the conditioned reinforcer signal $S_{\mathrm{x}} z_{\mathrm{x}_{f}}$ is much larger than $S_{\mathrm{x}} z_{\mathrm{x}}$; that is,

$$
S_{\mathrm{x} z \mathrm{x}_{f}} \gg S_{\mathrm{x} z \mathrm{x}_{r}} \text {. }
$$

When $\mathrm{CS}_{1}$ is turned on, $\mathrm{S}_{\mathrm{X}}$ tends to partially block activation of $S_{1}$ due to the strong positive feedback $S_{X} \rightarrow$ $\mathrm{D}_{f} \rightarrow \mathrm{S}_{\mathrm{X}}$ implied by Equation 51 . On the other hand, because $P\left(\mathrm{US} / \overline{\mathrm{CS}}_{1}\right)=.67$, attentional fluctuations during training trials enable the animal to attach fear reactions to only a subset of the sampled contextual cues $\mathrm{X}$. Thus in the moments just after presentation of $\mathrm{CS}_{1}, \mathrm{CS}_{1}$ may be sampled with neutral contextual cues and may thus begin to activate $S_{1}$. To the extent to which $S_{1}$ survives blocking by $S_{X}$, both sensory representations may share the limited-capacity STM activation, so that

$$
S_{1}+S_{\mathrm{X}}=S \text {, }
$$

although, due to blocking of $\mathrm{CS}_{1}$ by $\mathrm{X}$, often

$$
S_{\mathrm{X}}>S_{1} \text {. }
$$

When Equation 52 holds, the total signal $S_{1} z_{1 f}+S_{x} z_{f}$ to $\mathrm{D}_{f}$ is smaller than the total signal $S z_{x_{f}}$ to $\mathrm{D}_{f}$ that was active in response to $X$ alone. Consequently, a sudden decrease in total input to $D_{f}$ may occur after $C S_{1}$ is presented. This decrease causes a net reduction in conditioned fear and, if it is large enough, can cause a relief rebound at $D_{r}$. Thus $C_{1}$ can act as a conditioned inhibitor when it is presented within context $X$ whether or not $z_{1 r}$ is larger than $z_{1 f}$. The possibility that $S_{1}$ can act like a conditioned inhibitor even if $z_{1 f}>z_{1 r}$ depends critically upon the antagonistic rebound properties of the READ circuit. When a relief rebound does occur, there exists a range of parameters such that $S_{1}$ can become a source of net conditioned relief, by being associated with the rebound at $\mathrm{D}_{r}$.

Some more subtle effects should also be noted. By Equations 21 and $22,\left[x_{5}\right]^{+}>0$ only if $\left[x_{6}\right]^{+}=0$ and $\left[x_{6}\right]^{+}$ $>0$ only if $\left[x_{5}\right]^{+}=0$. On the other hand, $z_{k 7}$ and $z_{k 8}$ in Equations 14 and 15 perform a slow time-average of these quantities, as in Equation 38. Thus, although $\left[x_{5}\right]^{+}$and $\left[x_{6}\right]^{+}$cannot simultaneously be positive, $z_{k 7}$ and $z_{k 8}$ can both be positive in a probabilistically defined environment, such as $P\left(\mathrm{US} / \mathrm{CS}_{1}\right)=.33, P\left(\mathrm{US} / \overline{C S}_{1}\right)=.67$, wherein expectations are intermittently disconfirmed. In such a situation, presentation of $\mathrm{CS}_{1}$ can generate net positive signals $S_{1} z_{1 f}$ and $S_{1} z_{1 r}$ to both $\mathrm{D}_{f}$ and $\mathrm{D}_{r}$. When this happens,

$$
\frac{S_{1} z_{1 f}+S_{\mathrm{x} \mathrm{x}_{f}}}{S_{1} z_{1 r}+S_{\mathrm{x} \mathrm{x}_{r}}}<\frac{z_{\mathrm{x}_{f}}}{z_{\mathrm{x} r}}
$$

Hence, in addition to the decrease in total input to $\mathrm{D}_{f}$, there may also be an increase in the total input to $D_{r}$. When this occurs, the fearful difference $\Delta$ in Equation 34 may decrease as the adaption level $\hat{l}$ in Equation 33 increases. Thus a reduction in sensitivity to shock may occur in addition to, or in lieu of, a net relief reaction.

The same type of explanation shows why the critical factor in generating conditioned excitation or inhibition was whether or not unsignaled shocks were given in the conditioning context. Once a net fear connection $S_{1} \rightarrow D_{f}$ or a net relief connection $S_{1} \rightarrow D_{r}$ is established within the conditioning context, it is carried intact to the same or to a novel, test context. In addition, the associative averaging property defined by Equations 35 and 36 can explain why prior unsignaled shocks in context B do not necessarily increase the conditioned excitatory effect of $\mathrm{CS}_{\mathbf{1}}$ in context $\mathrm{B}$ during conditioning. 


\section{The Asymmetry Between Context Inflation and Context Deflation}

In the context deflation experiment, training on $P\left(\mathrm{US} / \mathrm{CS}_{1}\right)=.33, P\left(\mathrm{US} / \overline{\mathrm{CS}}_{1}\right)=.67$ was followed by extinction on $P(\mathrm{US} / \overline{\mathrm{CS}})=0$. Then $\mathrm{CS}_{1}$ was tested in both a deflated group and a nondeflated group. The lick latency to $\mathrm{CS}_{1}$ was longer after deflation than in the absence of deflation. This type of effect follows from the properties summarized in Section 30 . Without deflation, presentation of $\mathrm{CS}_{1}$ in $\mathrm{X}$ can reduce the fearful difference $\Delta$ and increase the adaptation level $\hat{I}$ relative to the dipole's state in response to $\mathrm{X}$ alone. After deflation, $\mathrm{X}$ does not generate fearful inputs to $D_{f}$. Hence the deflated $X$ does not establish a large fear reaction within $\mathrm{D}_{f}$, and thus does not block $\mathrm{CS}_{1}$. Presentation of $\mathrm{CS}_{1}$ in $\mathrm{X}$ can therefore effectively activate $S_{1}$, which can cause an increase in the fearful difference $\Delta$ relative to the dipole's state in response to $\mathrm{X}$ alone. Consequently, deflating the context $\mathrm{X}$ can increase the conditioned excitor properties of $C S_{1}$ when it is subsequently presented in $X$.

In the context inflation situation, $P(\mathrm{US} / \overline{\mathrm{CS}})$ is chosen to be larger than the $P(\mathrm{US} / \overline{\mathrm{CS}})=.67$ used during training trials, with little effect on $\mathrm{CS}_{1}$ 's effect during testing. Several model properties conspire to produce this result. These properties depend upon the fact that probabilities $P\left(\mathrm{US} / \overline{\mathrm{CS}}_{1}\right)$ that significantly exceed .67 define a learning situation wherein essentially all contextual cues $\mathrm{X}$ may be associated strongly with $\mathrm{D}_{f}$. The context $\mathrm{X}$ becomes one characterized by inescapable fear.

In such a situation, very strong, persistent activation of the $S_{X} \rightarrow D_{f} \rightarrow S_{X}$ pathway occurs. Thus $S_{1}$ is strongly blocked by $S_{X}$ whenever $C_{1}$ is presented. This property prevents $S_{1}$ from reading out the conditioned reinforcer values that could otherwise have enabled $\mathrm{CS}_{1}$ to act as a conditioned inhibitor. Choosing a very high $P\left(\mathrm{US} / \overline{C S}_{1}\right)$ can also persistently inhibit the orienting reactions (Grossberg, 1975, 1982c, 1984b) which could have enabled the model to intermittently reset fearful contextual representations and thereby facilitate the probability that initial processing of $\mathrm{CS}_{1}$ could partially overcome the strong contextual blocking effect.

\section{Some Additional Data Explanations}

To explain the data of Cotton et al. (1982), summarized in Section 21, it is necessary to add only that a large shock can cause large $z x_{f}$ values to be learned, whereas a small shock causes only small $z_{x_{f}}$ values to be learned. These conclusions follow from inspection of Equations 4, 8, 10, and 14 under the hypothesis that $J$ is large (small) when shock is large (small).

The fact that conditioned excitation is retarded and conditioned inhibition is facilitated by preconditioning USalone exposures (Hinson, 1982) can be explained by the same mechanisms that explained $\mathrm{CS}_{1}$ properties in response to $P\left(\mathrm{US} / \mathrm{CS}_{1}\right)=.33, P\left(\mathrm{US} / \overline{\mathrm{CS}}_{1}\right)=.67$, with the difference that the $P\left(\mathrm{US} / \overline{C S}_{1}\right)$ manipulation precedes, rather than being interspersed with, $\mathrm{CS}_{1}$ trials. The argument did not depend upon this difference.
The classical fact that a conditioned inhibitor attenuates excitor behavior on a summation test far more than does a novel stimulus (Pavlov, 1927) can be traced to the different types of mechanisms that regulate attention through an expectancy mismatch and through the $S \rightarrow D \rightarrow S$ feedback pathways. Whereas a novel event can amplify its initial storage in STM via an expectancy mismatch, and thereby temporarily remove attentional resources from a conditioned excitor, the conditioned excitor can restore its attentional focus through persistent self-amplification via its $S \rightarrow D_{f} \rightarrow S$ pathway subsequent to the novel event. In contrast, a conditioned inhibitor with a sufficiently strong $S \rightarrow D_{r} \rightarrow S$ pathway can compete effectively with the $S \rightarrow D_{f} \rightarrow S$ pathway of a conditioned excitor throughout the time interval of their simultaneous STM storage, thereby causing a sustained reduction in the efficacy of the conditioned excitor.

\section{Concluding Remarks}

At least four types of learning processes are relevant in the data discussions herein: learning of conditioned reinforcement, incentive motivation, conditioned response, and sensory expectancy. These several types of learning processes, which by their very nature operate on a slow time scale, regulate and are regulated by rapidly fluctuating limited-capacity STM representations of sensory events. The theory suggests how nonlinear feedback interactions among these fast information processing mechanisms and slow learning mechanisms control datapredictive emergent properties-such as STM reset, antagonistic rebound, adaptation level shift, associative averaging, and opponent extinction-which cannot be understood using traditional concepts such as contiguity learning and associative summation.

From this perspective, the different time scales on which sensory expectancies and conditioned reinforcers may be learned becomes a key explanatory issue. This is the type of distinction hinted at by Gibbon et al. $(1980$, p. 45), who observed, "Perhaps intermittent reinforcement generates schedule-induced cues only later in training, and early unreinforced trial episodes are in some sense 'ignored.",

\section{REFERENCES}

AdAms, J. A. (1967). Human memory. New York: McGraw-Hill. ALKoN, D. L. (1979). Voltage-dependent calcium and potassium ion conductances: A contingency mechanism for an associative learning model. Science, 205, 810-816.

ALKon, D. L. (1984a). Changes of membrane currents during learning. Journal of Experimental Biology, 112, 95-112.

AlKon, D. L. (1984b). Persistent calcium-mediated changes of identified membrane currents as a cause of associative learning. In D. $\mathrm{L}$. Alkon \& J. Farley (Eds.), Primary neural substrates of learning and behavioral change. New York: Cambridge University Press.

BaKer, A. G. (1974). Conditioned inhibition is not the symmetrical opposite of conditioned excitation: A test of the Rescorla-Wagner model. Learning \& Motivation, 5, 369-379.

BAnQuet, J.-P., \& Grossberg, S. (in press). Probing cognitive processes through the structure of event-related potentials during learning: An experimental and theoretical analysis. Applied Optics. 
Berger, T. W., \& Thompson, R. F. (1978). Neuronal plasticity in the limbic system during classical conditioning of the rabbit nictitating membrane response: I. The hippocampus. Brain Research, 145, 323-346.

BerLyne, D. E. (1969). The reward-value of indifferent stimulation. In J. T. Tapp (Ed.), Reinforcement and behavior. New York: Academic Press.

BindRA, D. (1968). Neurophysiological interpretation of the effects of drive and incentive-motivation on general activity and instrumental behavior. Psychological Review, 75, 1-22.

BOREN, J. J. (1961). Resistance to extinction as a function of the fixed ratio. Journal of Experimental Psychology, 61, 304-308.

BOTTJER, S. W . (1982). Conditioned approach and withdrawal behavior in pigeons: Effects of a novel extraneous stimulus during acquisition and extinction. Learning \& Motivation, 13, 44-67.

Bower, G. H. (1981). Mood and memory. American Psychologist, 36, 129-148.

Bower, G. H., Gilugan, S. G., Monteiro, K. P. (1981). Selectivity of learning caused by adaptive states. Joumal of Experimental Psychology: General, 110, 451-473.

BROGDEN, W. J. (1939). The effect of frequency of reinforcement upon the level of conditioning. Journal of Experimental Psychology, 24, 419-431

Bullock, D., \& Grossberg, S. (in press). Neural dynamics of planned arm movements: Emergent invariants and speed-accuracy properties during trajectory formation. Psychological Review.

CARPENTER, G. A., \& Grossberg, S. (1981). Adaptation and transmitter gating in vertebrate photoreceptors. Journal of Theoretical Neurobiology, 1, 1-42.

Carpenter, G. A., \& Grossberg, S. (1983). A neural theory of circadian rhythms: The gated pacemaker. Biological Cybemetics, 48, 35-59.

Carpenter, G. A., \& Grossberg, S. (1984). A neural theory of circadian rhythms: Aschoff's rule in diurnal and nocturnal mammals. American Journal of Physiology, 247, R1067-R1082.

CARPenter, G. A., \& Grossberg, S. (1985). A neural theory of circadian thythms: Split thythms, after-effects, and motivational interactions. Journal of Theoretical Biology, 113, 163-223.

Carpenter, G. A., \& GrossberG, S. (1986). Mammalian circadian rhythms: A neural network model. In G. A. Carpenter (Ed.), Some mathematical questions in biology: Circadian rhythms (Lectures on Mathematics in the Life Sciences, Vol. 19). Providence, RI: American Mathematical Society.

Carpenter, G. A., \& Grossberg, S. (1987). A massively parallel architecture for a self-organizing neural pattern recognition machine. Computer Vision, Graphics, \& Image Processing, 37, 54-115.

Carpenter, G. A., \& Grossberg, S. (in press a). Neural dynamics of category learning and recognition: Attention, memory consolidation, and amnesia. In J. Davis, R. Newburgh, \& E. Wegman (Eds.), Brain structure, learning, and memory (AAAS Symposium Series).

Carpenter, G. A., \& Grossberg, S. (in press b). Neural dynamics of category learning and recognition: Structural invariants, reinforcement, and evoked potentials. Quantitative Analyses of Behavior, 8.

CohEN, M. A., \& Grossberg, S. (1986). Neural dynamics of speech and language coding: Developmental programs, perceptual grouping, and competition for short term memory. Human Neurobiology, 5, 1-22.

Cohen, M. A., \& Grossberg, S. (1987). Masking fields: A massively parallel neural architecture for learning, recognizing, and predicting multiple groupings of pattemed data. Applied Optics, 26, 1866-1891.

Cotton, M. M., Goodall, G., \& Mackintosh, N. J. (1982). Inhibitory conditioning resulting from a reduction in the magnitude of reinforcement. Quarterly Journal of Experimental Psychology, 34B, $163-180$.

DAVENPORT, J. W. (1969). Successive acquisitions and extinctions of discrete bar-pressing in monkeys and rats. Psychonomic Science, 16, 242-244.

DENNY, M. R. (1970). Relaxation theory and experiments. In F. R. Brush (Ed.), Aversive conditioning and learning. New York: Academic Press.
DEVITo, P. L. (1980). The extinction of a conditioned fear inhibitor and conditioned fear excitor. Unpublished doctoral dissertation, University of Pittsburgh.

Domuan, M., \& Wilson, N. E. (1972). Specificity of cue to consequence in aversion learning in the rat. Psychonomic Science, 26 , 143-145.

Dunham, P. J. (1971). Punishment: Method and theory. Psychological Review, 78, 58-70.

Dunham, P. J., Mariner, A., \& ADAms, H. (1969). Enhancement of off-key pecking by on-key punishment. Journal of the Experimental Analysis of Behavior, 1, 156-166.

FELTON, M., \& LYON, D. O. (1966). The post-reinforcement pause Journal of the Experimental Analysis of Behavior, 9, 131-134.

ForeE, M., \& LoLoRdo, V. M. (1973). Attention in the pigeon Differential effects of food-getting versus shock-avoidance procedures. Journal of Comparative \& Physiological Psychology, 85, 551-558.

Garcia, J., \& KoELLING, R. A. (1966). Relation of cue to consequence in avoidance learning. Psychonomic Science, 4, 123-124.

Gibbon, J., Farrell, L., Locurto, C. M., Duncan, H. J., \& TerRACE, H. S. (1980). Partial reinforcement in autoshaping with pigeons. Animal Learning \& Behavior, 8, 45-59.

Gibes, C. M., Latham, S. B., \& Gormezano, I. (1978). Classical conditioning of the rabbit nictitating membrane response: Effects of reinforcement schedule on response maintenance and resistance to extinction. Animal Learning \& Behavior, 6, 209-215.

GonzaLEZ, F. A. (1973). Effects of partial reinforcement (25\%) in an autoshaping procedure. Bulletin of the Psychonomic Society, 2, 299-301.

Gonzalez, F. A. (1974). Effects of varying the percentage of key illuminations paired with food in a positive automaintenance procedure. Journal of the Experimental Analysis of Behavior, 22, 483-489.

Gonzalez, R. C., Berger, B. D., \& Bitterman, M. E. (1967). Improvement in habit-reversal as a function of amount of training per reversal and other variables. American Journal of Psychology, 79, 517-530.

Gormezano, I., Kehoe, E. J., \& Marshall, B. S. (1983). Twenty years of classical conditioning research with the rabbit. Progress in Psychobiology \& Physiological Psychology, 10, 197-275.

GrossBerg, S. (1968). Some physiological and biochemical consequences of psychological postulates. Proceedings of the National Academy of Sciences, 60, 758-765.

GrossberG, S. (1969a). On learning and energy-entropy dependence in recurrent and nonrecurrent signed networks. Journal of Statistical Physics, 1, 319-350.

GrossBerG, S. (1969b). On the production and release of chemical transmitters and related topics in cellular control. Joumal of Theoretical Biology, 22, 325-364.

GrossBERG, S. (1969c). Some networks that can learn, remember, and reproduce any number of complicated space-time patterns: I. Journal of Mathematics \& Mechanics, 19, 53-91.

GrossBerg, S. (1970). Some networks that can learn, remember, and reproduce any number of complicated space-time patterns: II. Studies in Applied Mathematics, 49, 135-166.

GrossberG, S. (1971). On the dynamics of operant conditioning. Joumal of Theoretical Biology, 33, 225-255.

GrossBerg, S. (1972a). A neural theory of punishment and avoidance: I. Qualitative theory. Mathematical Biosciences, 15, 39-67.

Grossberg, S. (1972b). A neural theory of punishment and avoidance: II. Quantitative theory. Mathematical Biosciences, 15, 253-285.

GrossBerg, S. (1973). Contour enhancement, short-term memory, and constancies in reverberating neural networks. Studies in Applied Mathematics, 52, 217-257.

GrossBerg, S. (1975). A neural model of attention, reinforcement, and discrimination learning. Intemational Review of Neurobiology, 18, 263-327.

GrossBerG, S. (1976a). Adaptive pattern classification and universal recoding: I. Parallel development and coding of neural feature detectors. Biological Cybernetics, 23, 121-134. 
Grossberg, S. (1976b). Adaptive pattern classification and universal recoding: II. Feedback, expectation, olfaction, and illusions. Biological Cybernetics, 23, 187-202.

GrossberG, S. (1978). A theory of human memory: Self-organization and performance of sensory-motor codes, maps, and plans. In R. Rosen \& F. Snell (Eds.), Progress in theoretical biology (Vol. 5, pp. 233-374). New York: Academic Press.

Grossberg, S. (1980). How does a brain build a cognitive code? Psychological Review, 87, 1-51.

GrossBerg, S. (1982a). Processing of expected and unexpected events during conditioning and attention: A psychophysiological theory. Psychological Review, 89, 529-572.

GrossBERG, S. (1982b). A psychophysiological theory of reinforcement, drive, motivation, and attention. Journal of Theoretical Neurobiology, 1, 286-369.

GrossBerg, S. (1982c). Studies of mind and brain: Neural principles of learning, perception, development, cognition, and motor control. Boston: Reidel Press.

GrossBerg, S. (1983). The quantized geometry of visual space: The coherent computation of depth, form, and lightness. Behavioral \& Brain Sciences, 6, 625-657.

GrossBerg, S. (1984a). Some normal and abnormal behavioral syndromes due to transmitter gating of opponent processes. Biological Psychiatry, 19, 1075-1118.

GROSSBERG, S. (1984b). Some psychophysiological and pharmacological correlates of a developmental, cognitive, and motivational theory. In R. Karrer, J. Cohen, \& P. Tueting (Eds.), Brain and information: Event related potentials. New York: New York Academy of Sciences.

GrossBerg, S. (1985). The hypothalamic control of eating and circadian rhythms: Opponent processes and their chemical modulators. In L. Rensing (Ed.), Temporal order. New York: Springer-Verlag.

Grossberg, S. (Ed.) (1987a). The adaptive brain: I. Cognition, learning, reinforcement, and rhythm. Amsterdam: Elsevier/North-Holland.

Grossberg, S. (Ed.). (1987b). The adaptive brain: II. Vision, speech, language, and motor control. Amsterdam: Elsevier/North-Holland.

GrossBerg, S. (1987c). Cortical dynamics of three-dimensional form, color, and brightness perception: I. Monocular theory. Perception \& Psychophysics, 41, 87-116.

Grossberg, S. (1987d). Cortical dynamics of three-dimensional form, color, and brightness perception: II. Binocular theory. Perception \& Psychophysics, 41, 117-158.

GrossBerG, S., \& GưowskI, W. (1987). Neural dynamics of decision making under risk: Affective balance and cognitive-emotional interactions. Psychological Review, 94, 300-318.

Grossberg, S., \& KUPERSTEIN, M. (1986). Neural dynamics of adaptive sensory-motor control: Ballistic eye movements. Amsterdam: Elsevier/North-Holland.

GrossBerg, S., \& LeVINE, D. S. (in press). Neural dynamics of attentionally modulated Pavlovian conditioning: Blocking, inter-stimulus interval, and secondary reinforcement. Applied Optics.

Grossberg, S., \& Mingolla, E. (1985). Neural dynamics of form perception: Boundary completion, illusory figures, and neon color spreading. Psychological Review, 92, 173-211.

GrossBerG, S., \& SCHMAJUK, N. (1987). Neural dynamics of anticipatory timing and temporal discrimination during classical conditioning. Manuscript in preparation.

GrossBerG, S. , \& STONE, G. O. (1986a). Neural dynamics of attention switching and temporal-order information in short-term memory. Memory \& Cognition, 14, 451-468.

GrossberG, S., \& Stone, G. O. (1986b). Neural dynamics of word recognition and recall: Attentional priming, learning, and resonance. Psychological Review, 93, 46-74.

HAMMOND, L. J. (1968). Retardation of fear acquisition by a previously inhibitory CS. Journal of Comparative \& Physiological Psychology, 66, 756-758.

Hawkins, R. D., Abrams, T. W., Carew, T. J., \& Kandel, E. R. (1983). A cellular mechanism of classical conditioning in Aplysia: Activity-dependent amplification of presynaptic facilitation. Science, 219, 400-405.
Hinson, R. E. (1982). Effects of UCS preexposure on excitatory and inhibitory rabbit eyelid conditioning: An associative effect of conditioned contextual stimuli. Journal of Experimental Psychology: Animal Behavior Processes, 8, 49-61.

Holland, P. C., \& Rescorla, R. A. (1975). Second-order conditioning with food unconditioned stimulus. Joumal of Comparative \& Physiological Psychology, 88, 459-467.

Holman, E. W. (1976). The effect of drug habituation before and after taste aversion leaming in rats. Animal Learning \& Behavior, 4, 329-332.

Jenkins, H. M., \& Lambos, W. A. (1983). Tests of two explanations of response elimination by noncontingent reinforcement. Animal Learning \& Behavior, 11, 302-308.

KAMIN, L. J. (1968). "Attention-like" processes in classical conditioning. In M. R. Jones (Ed.), Miami symposium on the prediction of behavior: Aversive stimulation. Miami: University of Miami Press.

Kamin, L. J. (1969). Predictability, surprise, attention, and conditioning. In B. A. Campbell \& R. M. Church (Eds.), Punishment and aversive behavior. New York: Appleton-Century-Crofts.

Kaufman, M. A., \& Bolles, R. C. (1981). A nonassociative aspect of overshadowing. Bulletin of the Psychonomic Society, 18, 318-320.

KilleEN, P. R. (1982a). Incentive theory. In D. J. Bernstein (Ed.), Nebraska symposium on motivation, 1981: Response structure and organization. Lincoln: University of Nebraska Press.

KILLEEN, P. R. (1982b). Incentive theory: II. Models for choice. Joumal of the Experimental Analysis of Behavior, 38, 217-232.

Kleiman, M. C., \& Fowler, H. (1984). Variations in explicitly unpaired training are differentially effective in producing conditioned inhibition. Learning \& Motivation, 15, 127-155.

LEVY, W, B. (1985). Associative changes at the synapse: LTP in the hippocampus. In W. B. Levy, J. Anderson, \& S. Lehmkuhle (Eds.), Synaptic modification, neuron selectivity, and nervous system organization. Hillsdale, NJ: Erlbaum.

Levy, W. B., Brassell, S. E., \& Moore, S. D. (1983). Partial quantification of the associative synaptic learning rule of the dentate gyrus. Neuroscience, 8, 799-808.

Levy, W. B., \& DeSmond, N. L. (1985). The rules of elemental synaptic plasticity. In W. B. Levy, J. Anderson, \& S. Lehmkuhle (Eds.), Synaptic modification, neuron selectivity, and nervous system organization. Hillsdale, NJ: Erlbaum.

LEYLAND, C. M. (1977). Higher-order autoshaping. Quarterly Journal of Experimental Psychology, 29, 607-619.

LYNCH, G. (1986). Synapses, circuits, and the beginnings of memory. Cambridge, MA: MIT Press.

LYSLE, D. T., \& FowLER, H. (1985). Inhibition as a "slave" process: Deactivation of conditioned inhibition through extinction of conditioned excitation. Journal of Experimental Psychology: Animal Behavior Processes, 11, 71-94.

Mackintosh, N. J. (1973). Stimulus selection: Learning to ignore stimuli that predict no change in reinforcement. In R. A. Hinde \& J. S. Hinde (Eds.), Constraints of learning. London: Academic Press.

Masterson, F. A. (1970). Is termination of a warning signal an effective reward for the rat? Journal of Comparative \& Physiological Psychology, 72, 471-475.

MCAllister, W. R., \& MCAluster, D. E. (1971). Behavioral measurement of conditioned fear. In F. R. Brush (Ed.), Aversive conditioning and learning. New York: Academic Press.

McAllister, W. R., MCAluster, D. E., \& Douglass, W. K. (1971). The inverse relationship between shock intensity and shuttlebox avoidance learning in rats. Journal of Comparative \& Physiological Psychology, 74, 426-433.

McCormick, D. A., \& Thompson, R. F. (1984). Cerebellum: Essential involvement in the classically conditioned eyelid response. Science, 223, 296-299.

MiLLER, N. E. (1963). Some reflections on the law of effect produce a new alternative to drive reduction. In M. R. Jones (Ed.), Nebraska symposium on motivation. Lincoln: University of Nebraska Press.

Miller, R. R., \& Schachtman, T. R. (1985). Conditioning context as an associative baseline: Implications for response generation and the nature of conditioned inhibition. In R. R. Miller \& N. E. Spear 
(Eds.), Information processing in animals: Conditioned inhibition. Hillsdale, NJ: Erlbaum.

OWRen, M. J., KaPLAN, P. S. (1981, April). On the failure to extinguish Pavlovian conditioned inhibition: A test of a reinstatement hypothesis. Paper presented at the meeting of the Midwestern Psychological Association, Detroit.

Pavlov, I. P. (1927). Conditioned reflexes. London: Oxford University Press.

Pearce, J. M., Hall, G. (1980). A model for Pavlovian learning: Variations in the effectiveness of conditioned but not of unconditioned stimuli. Psychological Review, 87, 532-552.

Perkins, C. C., JR., Beavers, W. O., Hancock, R. A., Jr., HemMENDinger, P. C., Hemmendinger, D., \& RiOCI, J. A. (1975). Some variables affecting rate of key pecking during response-independent procedures (autoshaping). Joumal of the Experimental Analysis of Behavior, 24, 59-72.

RASHOTTE, M. E., GRIFFin, R. W., \& SisK, C. L. (1977). Second-order conditioning of the pigeon's keypeck. Animal Learning \& Behavior, 5, 25-38.

Rauschecker, J. P., \& Singer, W. (1979). Changes in the circuitry of the kitten's visual cortex are gated by postsynaptic activity. $\mathrm{Na}$ ture, 280, 58-60.

Rescorla, R. A. (1968). Probability of shock in the presence and absence of CS in fear conditioning. Journal of Comparative \& Physiological Psychology, 66, 1-5.

RESCORLA, R. A. (1969). Establishment of a positive reinforcer through contrast with shock. Journal of Comparative \& Physiological Psychology, 67, 260-263.

Rescorla, R. A., \& LoLoRdo, V. M. (1965). Inhibition of avoidance behavior. Journal of Comparative \& Physiological Psychology, 69, 406-412.

Rescorla, R. A., \& Wagner, A. R. (1972). A theory of Pavlovian conditioning: Variations in the effectiveness of reinforcement and nonreinforcement. In A. H. Black \& W. F. Prokasy (Eds.), Classical conditioning: II. Current research and theory. New York: AppletonCentury-Crofts.

Reynierse, J. H., \& Rizley, R. C. (1970). Relaxation and fear as determinants of maintained avoidance in rats. Journal of Comparative \& Physiological Psychology, 72, 223-232.

Rizley, R. C., \& Rescorla, R. A. (1972). Associations in secondorder conditioning and sensory preconditioning. Journal of Comparative \& Physiologial Psychology, 81, 1-11.

Schmaltz, L. W., \& Theios, J. (1972). Acquisition and extinction of a classically conditioned response in hippocampectomized rabbits (Oryctolagus cuniculus). Joumal of Comparative \& Physiological Psychology, 79, 328-333.

SchwarTz, B., \& Williams, D. R. (1972). Two different kinds of keypeck in the pigeon: Some properties of responses maintained by negative and positive response-reinforcer contingencies. Joumal of the Experimental Analysis of Behavior, 18, 201-216.

Seligman, M. E. P., HAGER, I. L. (1972). Biological boundaries of learning. New York: Appleton-Century-Crofts.

SIEGEL, S., \& DomJAN, M. (1971). Backward conditioning as an inhibitory procedure. Learning \& Motivation, 2, 1-11.

SINGER, W. (1983). Neuronal activity as a shaping factor in the selforganization of neuron assemblies. In E. Basar, H. Flohr, H. Haken, \& A. J. Mandell (Eds.), Synergetics of the brain. New York: SpringerVerlag.

Solomon, R. L. (1980). The opponent-process theory of acquired motivation. American Psychologist, 35, 691-712.

Solomon, R. L. (1982). The opponent processes in acquired motivation. In D. W. Pfaff (Eds.), The physiological mechanisms of motivation. New York: Springer-Verlag.
Solomon, R. L., \& CoRBIT, J. D. (1974). An opponent process theory of motivation: I. Temporal dynamics of affect. Psychological Review, 81, 119-145.

SutTon, R. S., \& Barto, A. G. (1981). Toward a modern theory of adaptive networks: Expectation and prediction. Psychological Review, 88, $135-170$.

WASSERMAN, E. A. (1974). Stimulus-reinforcer predictiveness and selective discrimination learning in pigeons. Journal of Experimental Psychology, 103, 284-297.

Wasserman, E. A., Deich, J. D., Hunter, N. B., \& Nagamatsu, L. S. (1977). Analysing the random control procedure: Effects of paired and unpaired CS's and US's on autoshaping the chick's key peck with heat reinforcement. Learning \& Motivation, 8, 467-487.

Wasserman, E. A., Hunter, N. B., Gutowski, K. A., \& Bader, S. A. (1975). Autoshaping chicks with heat reinforcement: The role of stimulus-reinforcer and response-reinforcer relations. Journal of Experimental Psychology: Animal Behavior Processes, 104, 158-169.

Weisman, R. G., \& LiTner, J. S. (1969). The course of Pavlovian extinction and inhibition of fear in rats. Journal of Comparative \& Physiological Psychology, 69, 667-672.

WERBLIN, F. S. (1971). Adaptation in a vertebrate retina: Intracellular recordings in Necturus. Journal of Neurophysiology, 34, 228-241.

WITCHER, E. S. (1978). Extinction of Pavlovian conditioned inhibition. Unpublished doctoral dissertation, University of Massachusetts, Amherst.

Zimmer-Hart, C. L., \& Rescorla, R. A. (1974). Extinction of Pavlovian conditioned inhibition. Journal of Comparative \& Physiological Psychology, 86, 837-845.

\section{NOTES}

1. In addition to being used to analyze data about conditioning, specialized gated dipoles have been used to analyze data about certain mental disorders (Grossberg, 1972b, 1984a, 1984b), mammalian circadian rhythms (Carpenter \& Grossberg, 1983, 1984, 1985, 1986), eating and drinking rhythms (Grossberg, 1984b, 1985), photoreceptor transduction (Carpenter \& Grossberg, 1981), cortical mechanisms of preattentive visual perception (Grossberg, 1987c, 1987d; Grossberg \& Mingolla, 1985), decision making under risk (Grossberg \& Gutowski, 1987), and neural and evoked potential substrates underlying the learning of $\operatorname{cog}$ nitive recognition codes (Banquet \& Grossberg, in press; Carpenter \& Grossberg, 1987, in press a; Grossberg, 1980, 1984b). Many of these data have, moreover, received no other mechanistic explanation.

2 . The theory developed by Grossberg $(1975,1982 a)$ provided a realtime explanation of blocking and unblocking, as well as a variety of other conditioning properties such as overshadowing, partial reinforcement acquisition effect, secondary reinforcement, latent inhibition, superconditioning, learned helplessness, vicious circle behavior, hypothalamic self-stimulation, hyperphagic eating, intragastric drinking, peak shift and behavioral contrast, differential effects of sudden versus gradual shock onsets, and dishabituation by novel cues (Grossberg, 1971, 1972a, 1972b, 1982a, 1984b). Grossberg and Levine (in press) have further developed the theory by carrying out real-time computer simulations of blocking, inverted $U$ in learning as a function of interstimulus interval, anticipatory conditioned responses, secondary reinforcement, attentional focusing by conditioned motivational feedback, and limitedcapacity STM processing.

(Manuscript received March 6, 1987; revision accepted for publication August 17, 1987.) 